\title{
Carbon Nanotubes as Fluorescent Probes in Living Drosophila Embryos
}

\section{DISSERTATION}

zur Erlangung des mathematisch-naturwissenschaftlichen Doktorgrades

\section{"Doctor rerum naturalium"}

der Georg-August-Universität Göttingen

im Promotionsprogramm / Promotionsstudiengang Physik

der Georg-August-University School of Science (GAUSS)

vorgelegt von

\section{Constantin Dominic Clemens Kohl}

aus Karlsruhe

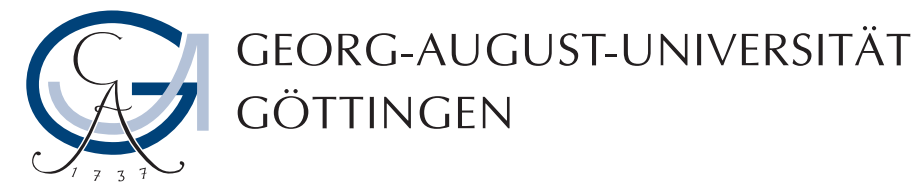

Göttingen, 2020 


\section{Betreuungsausschuss:}

- Prof. Dr. Christoph F. Schmidt, Drittes Physikalisches Institut, Georg-August-Universität Göttingen

- Prof. Dr. Stefan Klumpp, Institut für Dynamik Komplexer Systeme, Georg-August-Universität Göttingen

\section{Mitglieder der Prüfungskommission:}

- Referent: Prof. Dr. Christoph F. Schmidt, Drittes Physikalisches Institut, Georg-August-Universität Göttingen

- Korreferent: Prof. Dr. Stefan Klumpp, Institut für Dynamik Komplexer Systeme, Georg-August-Universität Göttingen

\section{Weitere Mitglieder der Prüfungskommission:}

- Prof. Dr. Burkhard Geil, Institut für Physikalische Chemie, Georg-August-Universität Göttingen

- Prof. Dr. Martin Göpfert, Schwann-Schleiden-Forschungszentrum, Abteilung: Zelluläre Neurobiologie, Georg-August-Universität Göttingen

- Dr. Florian Rehfeldt, Drittes Physikalisches Institut, Georg-August-Universität Göttingen

- Dr. Sebastian Kruss, Institut für Physikalische Chemie, Georg-August-Universität Göttingen 


\section{Erklärung}

Ich versichere wahrheitsgemäß, die Arbeit selbstständig angefertigt, alle benutzten Hilfsmittel vollständig angegeben und alles kenntlich gemacht zu haben, was aus Arbeiten anderer unverändert oder mit Abänderungen entnommen wurde.

Göttingen, den 10.2.2020 



\section{Contents}

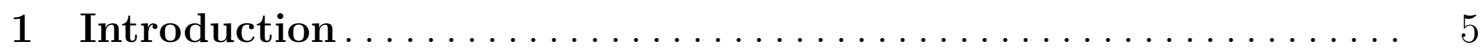

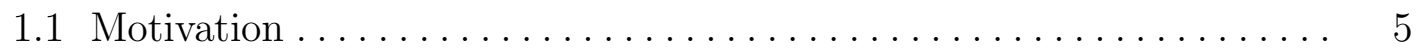

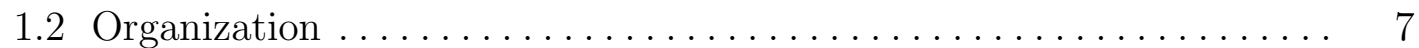

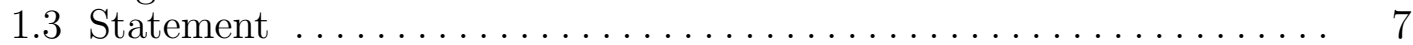

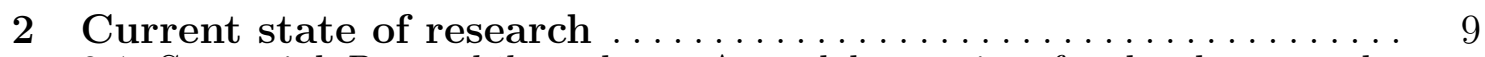

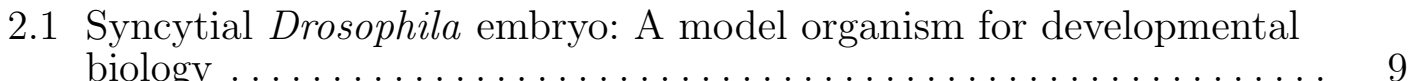

2.2 Carbon nanotubes . . . . . . . . . . . . . . . . . . . . . 15

2.2.1 Carbon nanotubes as fluorescent probes in living organisms. . . . 15

2.2 .2 Carbon nanotube geometry and fluorescence . . . . . . . . . 17

3 Materials and sample preparation $\ldots \ldots \ldots \ldots \ldots \ldots \ldots \ldots \ldots$

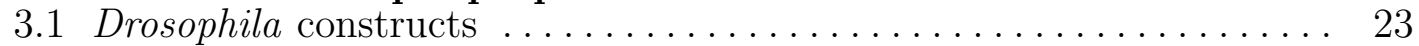

3.2 Preparing and functionalization of carbon nanotubes ........... 24

3.2.1 Suspending carbon nanotubes in watery environments . . . . . 25

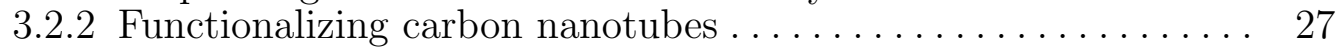

3.3 Preparation of Drosophila embryos $\ldots \ldots \ldots \ldots \ldots \ldots \ldots \ldots \ldots \ldots \ldots$

3.3.1 Collection of Drosophila eggs . . . . . . . . . . . . . . . . . 28

3.3.2 Microinjecting carbon nanotube suspensions into Drosophila embryos $\ldots \ldots \ldots \ldots \ldots \ldots \ldots \ldots \ldots \ldots \ldots \ldots \ldots \ldots \ldots \ldots \ldots \ldots \ldots, 31$

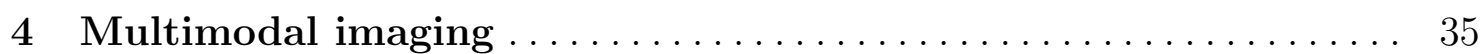

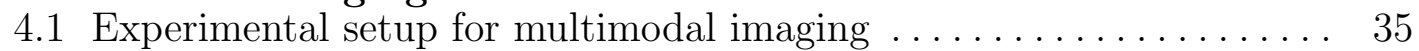

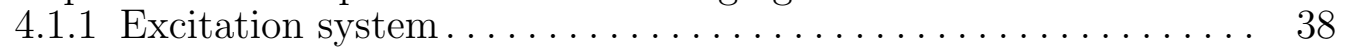

4.1 .2 Detection system $\ldots \ldots \ldots \ldots \ldots \ldots \ldots \ldots \ldots \ldots \ldots \ldots \ldots$

5 Characterizing optical properties of carbon nanotubes in living

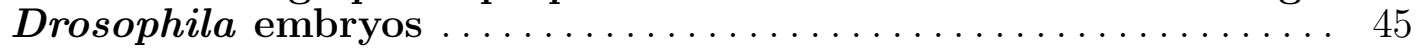

5.1 Excitation of carbon nanotube fluorescence in living Drosophila embryos 45

5.2 Point spread functions of carbon nanotubes in living Drosophila

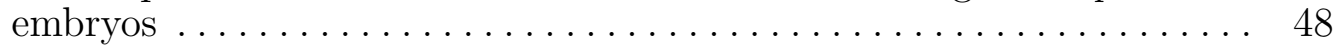

6 Dynamics of carbon nanotubes in Drosophila embryos . . . . . . . . 51

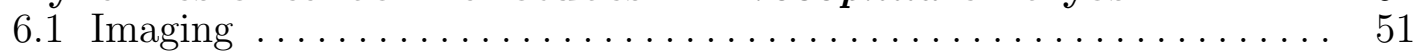

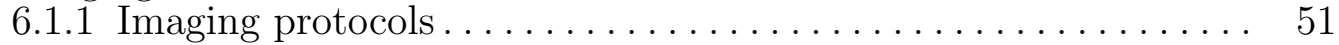

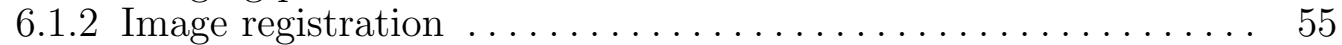

\begin{tabular}{|ll}
\hline 6.2 Tracking of individual carbon nanotubes in living Drosophila embryos 61 \\
\hline
\end{tabular}

6.3 Single-nanoparticle dynamics of fluorescent carbon nanotubes in

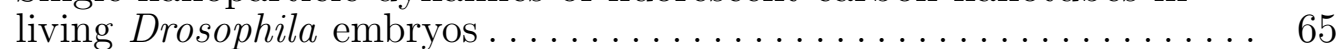

6.3 .1 Mean squared displacements . . . . . . . . . . . . . . . 65 


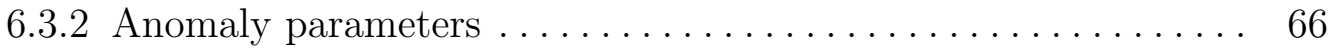

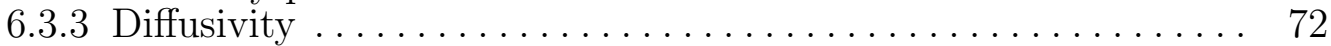

6.3.4 Velocity cross-correlations of individual carbon nanotube trajectories in living Drosophila embryos $\ldots \ldots \ldots \ldots \ldots \ldots .72$

\begin{tabular}{|}
6.3 .5 Hydrodynamic length of carbon nanotubes in living Drosophila \\
embryos $\ldots \ldots \ldots \ldots \ldots \ldots \ldots \ldots \ldots \ldots \ldots \ldots \ldots \ldots \ldots \ldots \ldots \ldots \ldots$ \\
\hline
\end{tabular}

6.4 Mesoscopic bulk dynamics: Particle image velocimetry with

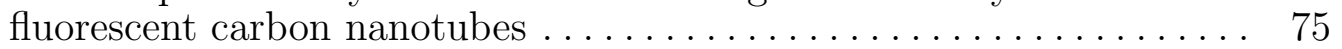

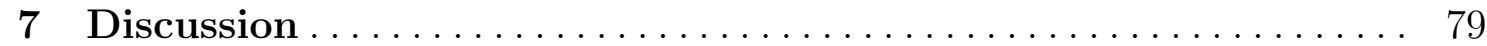

7.1 Carbon nanotube preparation and microinjection . . . . . . . . . . 79

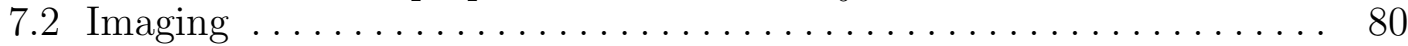

7.3 Optical properties of carbon nanotubes in living Drosophila embryos. 82

7.4 Dynamics of carbon nanotubes in Drosophila embryos $\ldots \ldots \ldots \ldots 83$

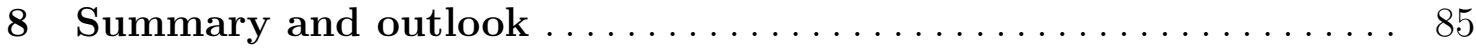

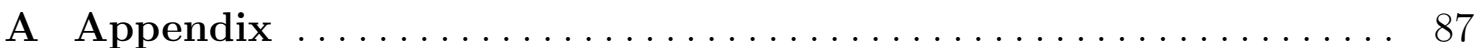

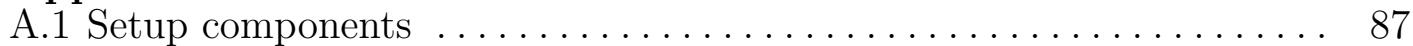

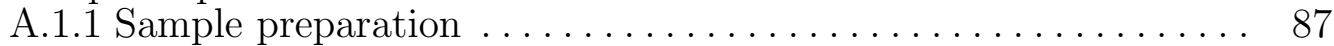

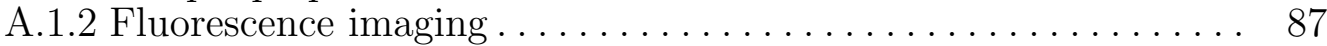

A.2 Chemical agents and disposables . . . . . . . . . . . . . . . . . 89

A.2.1 Carbon nanotube preparation . . . . . . . . . . . . . . . . 89

A.2.2 Fluorescence imaging. . . . . . . . . . . . . . . . . . . . . . 89

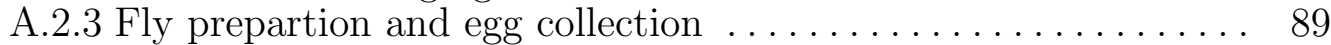

Acknowledgements. . . . . . . . . . . . . . . . . . . . . . 91

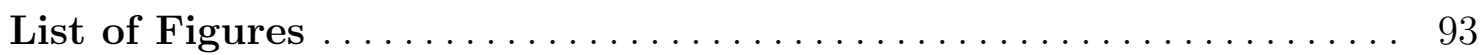

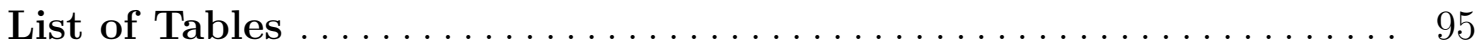

References ..................................... 97

Peer-reviewed journal publication related to this thesis . . . . . . . . . 109 


\section{List of abbreviations}

\begin{tabular}{|c|c|}
\hline $1 \mathrm{D}$ & one-dimensional \\
\hline $2 \mathrm{D}$ & two-dimensional \\
\hline ADP & adenosine diphosphate \\
\hline ARC & anti-reflective coating \\
\hline ATP & adenosine triphosphate \\
\hline CCD & charge-coupled device \\
\hline CMOS & complementary metal-oxide-semiconductor \\
\hline CNT & carbon nanotube \\
\hline CV(RMSE) & coefficient of variation of the root-mean-square error \\
\hline Drosophila & Drosophila melanogaster \\
\hline DMSO & dimethyl sulfoxide \\
\hline DNA & deoxyribonucleic acid \\
\hline DOS & density of states \\
\hline EGFP & enhanced green fluorescent protein \\
\hline EMCCD & electron multiplying charge-coupled device \\
\hline FOV & field of view \\
\hline G1 phase & gap 1 phase \\
\hline G2 phase & gap 2 phase \\
\hline His-EGFP & Histone2Av-EGFP \\
\hline His-EGFP/kin-5-Halo & enhanced green fluorescent protein with kinesin-5-HaloTag ${ }^{\circledR}$ \\
\hline InGaAs & indium gallium arsenide \\
\hline IR & infrared \\
\hline M phase & mitotic phase \\
\hline MAE & mean absolute error \\
\hline MSD & mean squared displacement \\
\hline MWCO & molecular weight cut-off \\
\hline NIR & near-infrared \\
\hline nRMSE & normalized root-mean-square error \\
\hline OD & optical density \\
\hline PIV & particle image velocimetry \\
\hline PSF & point spread function \\
\hline RMSE & root-mean-square error \\
\hline S phase & synthesis phase \\
\hline ssDNA & single-stranded deoxyribonucleic acid \\
\hline SSE & succinimidyl ester \\
\hline Ti:Sapph & titanium-sapphire \\
\hline VIS & visible \\
\hline
\end{tabular}





\section{Abstract}

In this project, a promising imaging method using near-infrared (NIR) fluorescent, DNA -wrapped carbon nanotubes (CNTs) is applied to study their optical and dynamical behavior in syncytial Drosophila melanogaster (Drosophila) embryos. Drosophila (commonly known as vinegar fly) is one of the most studied model organisms in developmental biology and increasingly draws attention from physical sciences. In hours-long syncytial stage of embryonic development (embryogenesis), nuclei in embryonic Drosophila form a highly dynamic 2D cortical layer unveiling a multitude of interesting dynamics. Capturing details of microscopic mechanics during embryogenesis on short time scales during such long measurement times pushes demands for both single-molecule and single-nanoparticle fluorescence experiments to their boundaries. Semiconducting NIR fluorescent CNTs are promising novel fluorescent markers for in vivo studies, since they have unique optoelectronic properties. They display extraordinary photostability and Stokes shifts that can reach several hundred nanometers, having an extended excitation spectrum in the visible (VIS) range. The photostable and intermittency-free NIR fluorescence of CNTs enable us to capture high frequency information of individual CNT trajectories in the living embryos, making CNTs valuable probes for long-time tracking over multiple division cycles inside living Drosophila embryos. We solubilize the hydrophobic CNTs in watery solutions and use biochemical linking methods to potentially assess specific binding of fluorescent CNTs to single kinesin-5 molecules in transgenic Drosophila embryos. With microinjection we introduce DNA-wrapped CNTs into syncytial Drosophila embryos of two transgenic types.

Here, we present a custom-built setup, allowing simultaneous imaging of CNT NIR fluorescence and enhanced green fluorescent protein (EGFP)-tagged nuclear histones. With an infrared (IR) spectrometer integrated into this VIS and NIR wide-field fluorescence microscope setup, we characterize the excitation spectrum of the CNTs used in our experiments to find the optimal wavelength for excitation of CNT NIR fluorescence. During measurements, we combine high frequency CNT NIR signals with corresponding low frequency nuclear Histone2Av-EGFP (His-EGFP) signals. This combination of two imaging channels provides a powerful tool for conducting single-nanoparticle experiments in vivo with CNTs correlated with EGFP labeling in the VIS channel over a wide time range, enabling us to simultaneously capture intracellular dynamics on multiple time scales. Within each of these embryos, we observe individual CNT fluorescence signals and VIS fluorescence of EGFP in nuclear 
histones during various division cycle phases in the cortical layer. We superimpose these fluorescence signals measured in different transgenic types of Drosophila for qualitative spatio-temporal orientation. Furthermore, we analyze dynamics of functionalized CNTs in the cortex of living embryos of different transgenic Drosophila. Information about intracellular dynamics of CNTs in these fly types is obtained by single-nanoparticle tracking and subsequent correlation analyses of individual CNTs. From these results, we infer dominant diffusive and sub-diffusive behavior of CNTs in the investigated embryos and find the hydrodynamic length of CNTs. Furthermore, we demonstrate quantitative results obtained from systematic imaging in embryonic tissue. In addition, we use NIR fluorescent CNTs in conjunction with particle image velocimetry to capture mesoscopic bulk dynamics of the cytoplasmic flow in developing Drosophila embryos. 


\section{Introduction}

\subsection{Motivation}

The non-equilibrium character of the environment of single-molecule dynamics in living system has gained great interest over the last decade [1 8]. A primal example for out-of-equilibrium systems are living organisms. Single-molecule experiments are the basis of understanding complex processes in living systems on a microscopic level. Although similar information can be obtained from simplified isolated systems, studying single-molecule dynamics in their natural environment, like cells or tissue, provide a more realistic view into the rich emergent properties of living matter [9 12]. Single-molecule and single-nanoparticle tracking using fluorescence microscopy are proven methods to understand biological functions in living organisms at microscopic level [7, 13. However, photobleaching and intermittency impede the tracking of single or small numbers of fluorophores. Furthermore, both single-molecule and single-nanoparticle experiments require high demands on experimental conditions, such as sample volume confinement and/or use of nano- or even picomolar concentrations of fluorescent tracers to reduce background effects [6, 7]. Conventional dyes such as enhanced green fluorescent protein (EGFP) emit in the visible range with relatively low Stokes shifts of typically tens of nanometers [14-16]. The hence limited signal-to-noise ratio combined with rapid photobleaching of those dyes demand a decision between high temporal resolution imaging and overall recording time. Carbon nanotubes (CNTs) are novel fluorescent probes with fascinating optoelectronic properties proven to be applicable in living organisms [6, 17 19]. Their high photostability, near-infrared (NIR) emission suppressing autofluorescence in cells and tissue combined with a large Stokes shift with excitation from the visible into the NIR range make CNTs a superior tool for in vivo fluorescence imaging [6, 20]. We thus exploit the fluorescence properties of CNTs to bypass the problems associated with conventional dyes.

In this work we combine the exceptional fluorescence properties of CNTs with the Drosophila melanogaster (Drosophila), a standard model organism in developmental biology. Their short nuclear replication cycles and straightforward handling make Drosophila embryos a prevalent model organism for non-equilibrium studies [21 24]. Recent studies used CNTs to specifically label individual kinesin-1 molecules in living cells. Here we extend this method to more complex systems. We investigate the feasibility of this promising method for marking one of the major players during 
nuclear replication, the kinesin-5 motor protein, in Drosophila embryos. Moreover, we observe the dynamics of CNTs in the highly dynamic two-dimensional (2D) cortex of Drosophila embryos over successive nuclear division stages, while taking advantage of the above-mentioned high temporal resolution. Furthermore, we investigate how the fluorescence of CNTs behaves in various tissue depths.

In addition, we use them as fluorescent tracers for non-specific probing of cytoplasm during its enhanced flow phase. We use the conventional dye EGFP to mark nuclear histones for spatio-temporal categorization of these dynamics. In our work we track individual CNTs in early Drosophila embryos over successive nuclear replication cycles in the embryos' syncytial stage. We present a custom-built wide-field microscopy setup, enabling us to simultaneously image conventional fluorescent dyes and NIR fluorescent CNTs in embryos of two different transgenic types. 


\subsection{Organization}

- Chapter 1 motivates this work and gives an overview of the scope of this thesis. It also informs about the contributions of researchers involved in this work.

- Chapter 2 displays the current state of research. State-of-the-art techniques and fundamentals necessary to understand this work are presented.

- Chapter 3 presents the materials used in this work: among other both the Drosophila melanogaster (Drosophila) fly and carbon nanotube (CNT) preparation. In this chapter, we also address techniques for suspending CNTs in watery solutions and for functionalizing CNT samples. This chapter also covers the topic of how CNT suspensions are injected into Drosophila embryos.

- Chapter 4 covers the experimental setup used during the multimodal fluorescence imaging. Large parts of this chapter are submitted to the journal Review of Scientific Instruments: "Near-infrared fluorescence imaging of single-walled carbon nanotubes in living organisms".

- Chapter 5 characterizes the optical and fluorescent properties of CNT samples used in this work. Furthermore, we present point spread functions (PSFs) of individual CNTs in living Drosophila embryos.

- Chapter 6 displays the imaging protocols used for studying dynamics in Drosophila embryos. Moreover, this chapter shows results obtained from particle tracking and presents the results exhibiting the transport behavior of fluorescent CNTs in two different genetic modifications of Drosophila embryos. Additionally, this chapter shows results obtained from particle image velocimetry (PIV) studies.

- Chapter 7 provides a discussion of the previous chapters.

- Chapter 8 gives an outlook and concludes the thesis.

\subsection{Statement}

Parts of this thesis are in progress to be published in peer-reviewed journals.

Where it seemed redundant, the author of this thesis, Constantin Kohl, decided to fully use the text from the submitted journal publication (see chapter 8). This concerns especially chapters 3.3, 4, 5. Constantin Kohl wrote the manuscripts of the draft of the submitted peer-reviewed journal publication linked to this work. The manuscript has been edited by Prof. Dr. Christoph F. Schmidt.

The details on the flies used in this work and in the submitted publication are the courtesy of Dr. Zhiyi Lv. The procedure for fly peparation portrayed in chapter 3.3 is the result of discussions with Dr. Zhiyi Lv.

The CNT suspensions leading to results within Drosophila embryos and being presented in this work have been produced by Dr. Kengo Nishi, if not stated otherwise. Constantin Kohl contributed to optimizing the suspension and functionalization procedure of CNTs.

The flies used for the data presented in this work were cultured by Dr. Zhiyi Lv.

Dr. Zhiyi Lv also microinjected CNT suspension into of Drosophila flies.

The data presented in this work has been acquired and analyzed by Constantin

Kohl. The measurement protocols were developed by Constantin Kohl. 



\section{Current state of research}

\subsection{Syncytial Drosophila embryo: A model organism for developmental biology}

Easy access to molecular processes in Drosophila and its fast reproduction rate has justified Drosophila to become one of the most important and most studied model organisms in developmental biology [23-26].

In the stage of syncytial blastoderm, the nuclei of the developing embryo are enclosed by a shared plasma membrane (Fig. 2.1) and (Fig. 2.2). From interphase 9 on nuclei move from the yolk towards the cortex, an active network of cytoskeletal filaments and native molecular motors, such as kinesin-5 (Fig. 2.1). At this point, the cortex forms a highly dynamic $2 \mathrm{D}$ material far away from thermodynamic equilibrium (Fig. 2.1).

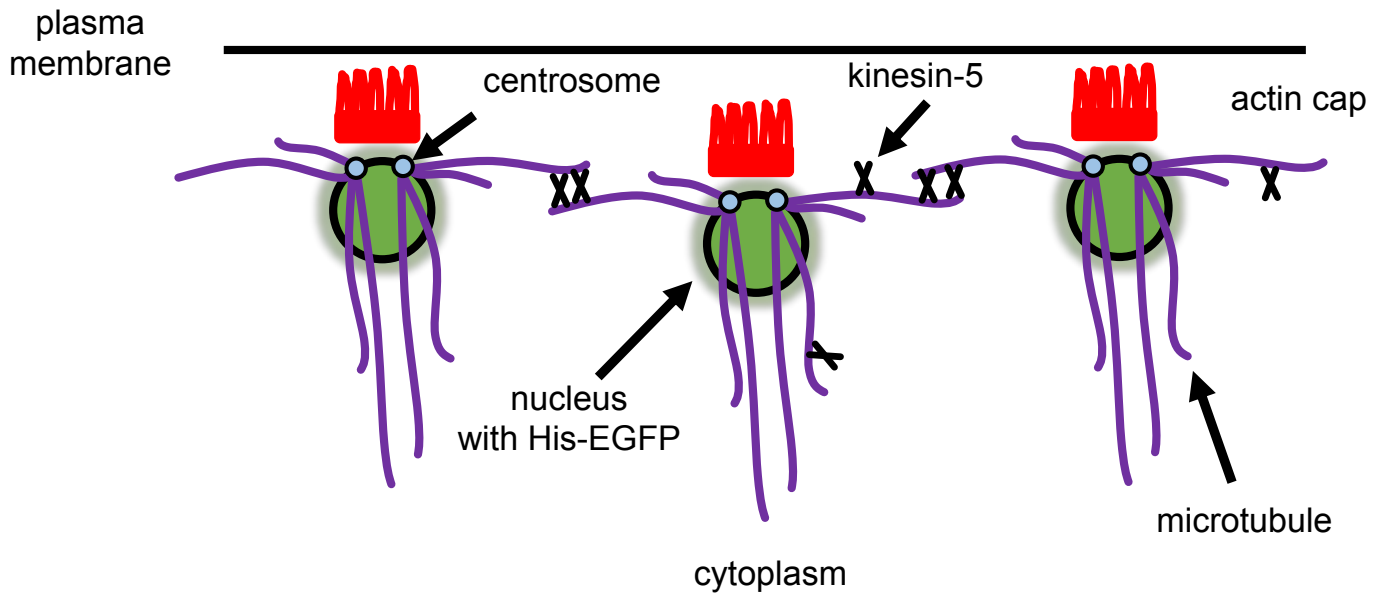

Figure 2.1. Schematic of the cortical region in syncytial Drosophila embryos. Modified after [27.

Individual nuclei interact with each other over several nuclear division cycles (interphase 9 to interphase 13) via a network consisting of cytoskeletal filaments, such as microtubules.

In this work, we focus on the cortical region during successive nuclear division phases in different developmental stages (Fig. 2.2). We also observe dynamics of the cytoplasm during a phase of increased cytoplasmic flow during syncytial blastoderm 
(Fig. 2.1).

Both systems, the 2D cortex and the flowing cytoplasm, in Drosophila provide an easily accessible primal example to study properties of self-organizing materials and collective phenomena in natural non-equilibrium systems with possible biomedical applications.

Division cycles in Drosophila embryos are reported to be in the range of 8 to 180 min each [28], while in mammalian cell lines such as HeLa cells a single division cycle needs up to $22 \mathrm{~h}$ for completion [29]. We could confirm that a division cycle in Drosophila embryos is in the order of about 15 to $30 \mathrm{~min}$. Drosophila embryos are usually about $500 \mu \mathrm{m}$ long [21, 30, 31] and measure $180 \mu \mathrm{m}$ [31] in width [30] (Fig. 2.2 .

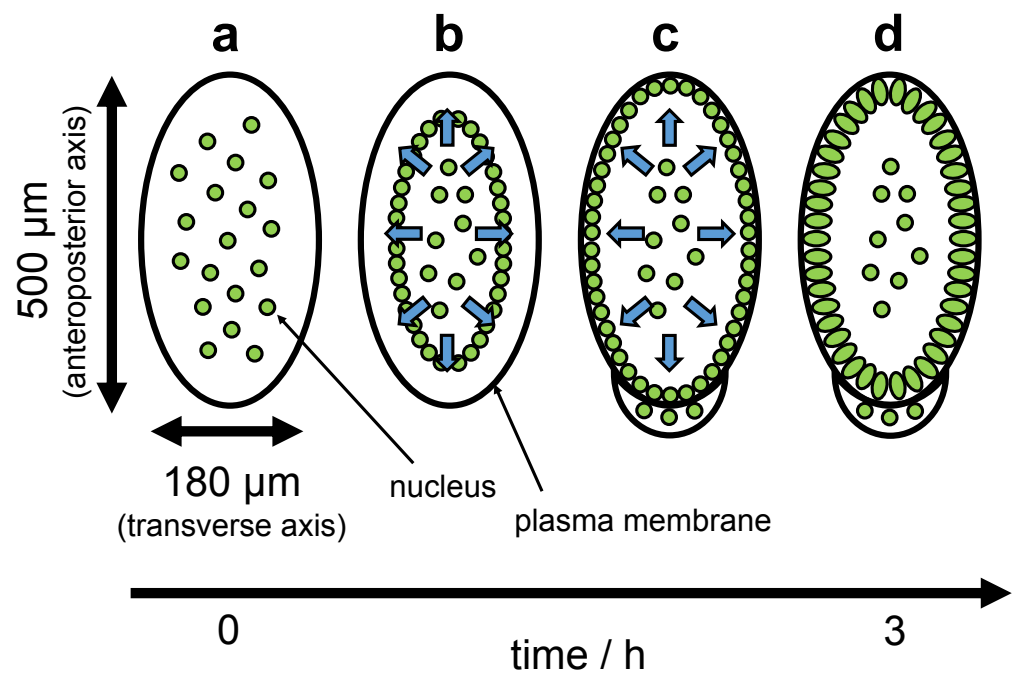

Figure 2.2. Schematic of developmental phases of syncytial Drosophila embryos. Illustrations modified after 32 34. (a) At the stage of early syncytial blastoderm, nuclei multiply nine times in division cycles 1 to 9 [32, 33. (b) Beginning nuclear migration towards periphery (blue arrows) after nine division cycles. 32, 33. (c) Outwardly directed nuclear migration continues and furrow canals encapsulate nuclei until concluding division cycle 13 [32, 33, 35, 36]. (d) In cellularization, epithelial cells formate, leaving behind encaged nuclei $32,33,37$.

Every living nucleus reproduces a finite amount of division cycles, the so called nuclear division cycles, during its life [38. Each nuclear division cycle can be separeted into two phases and five distinct sub-phases: interphase, consisting of gap 1 phase (G1 phase, synthesis phase (S phase, gap 2 phase (G2 phase and the mitotic phase (M phase) which is defined by mitosis and cytokinesis. M phase in turn can be divided into five morphologically distinct stages: prophase, prometaphase, metaphase, anaphase, telophase [38] (Fig. 2.3). 


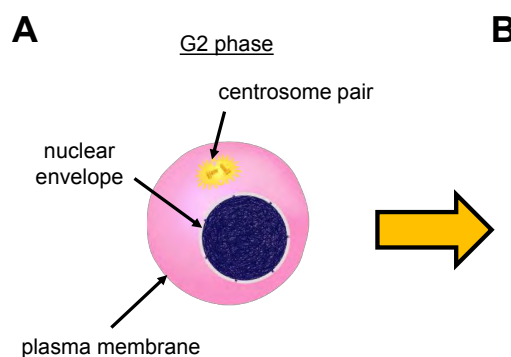

B

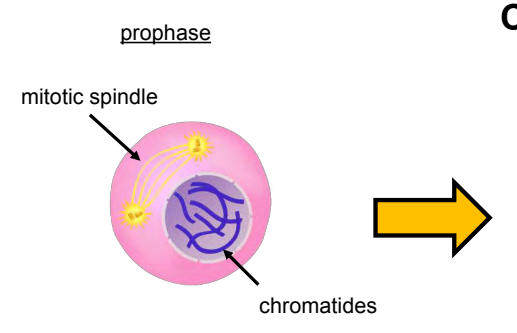

C prometaphase

D

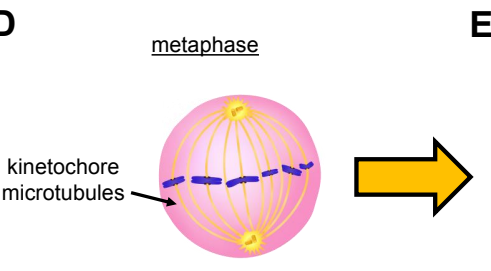

E

anaphase

$\mathbf{F}$

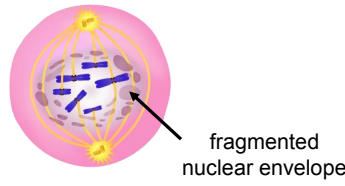

Figure 2.3. Schematic of G2 phase (A) and M phase (B)-(F). Modified after 39].

\section{Interphase}

The phase where nuclei prepare for the next division is called interphase. This phase can be divided into three stages: G1 phase, S phase and G2 phase 38, 40, 42. During S phase chromosomale deoxyribonucleic acid (DNA) is copied and centrosomes, the microtubule organization centers, are replicated. The S phase is framed by two monitoring phases, the gap phases (G1 and G2). In the G1 phase it is determined if the conditions outside the cell are beneficial enough to proceed to the S phase, After the successful completion of the $\mathrm{S}$ phase the G2 phase follows (Fig. 2.3, A). This phase will continue until possible DNA damages are repaired and until DNA replication is completed. During the gap phases, cytoplasmic organelles are duplicated while cell growth goes on [38]. In interphase, nuclei in Drosophila undergo a drift of about two nuclear diameters (about $10 \mu \mathrm{m}$ ) (Chap. 6.4).

\section{M phase}

M phase summarizes mitosis and cytokinesis [38]. During mitosis, the actual division of the mother nucleus into two genetically identical daughter nuclei takes place. Mitosis itself passes five morphologically distinct phases [38]: prophase, prometaphase, metaphase, anaphase and telophase (Fig. 2.3, B-F). During prophase chromosome condensation takes place in the nucleus. The mitotic spindle begins to form while the centrosomes separate. In the next mitotic phase, the prometaphase, the nuclear envelope collapses, enabling chromsome attachment to the spindle. During the following metaphase, the chromosomes align equatorially and microtubules attach to opposite spindle poles. At anaphase, chromosomes split up supported by outwardly directed movement of the spindle poles and shortening of microtubules attached to chromosomes (kinetochore microtubules). At telophase, the last phase of mitosis, the chromosomes end up at respective spindle poles, followed by the formation of a new nuclear envelope which packs the chromosmes. After telophase, cytokinesis 
concludes M phase by leaving behind two daughter nuclei being cleft off from the mother nucleus by an contractile acto-myosin ring [38].

To gain information about the micromechanical structure of the cortex to find the right stage for the single-nanoparticle experiments, it is crucial to discriminate interphases in the syncytial blastoderm (interphase 9 to 13) with strongly correlated nuclei from the cellular blastoderm (from interphase 14) with nuclei encaged by acto-myosin [32, 33, 37. To discriminate the different interphases during embryonic development, we use the number of nuclei per standardized area of $250 \mu \mathrm{m}$. The number of nuclei doubles with each interphase. Starting from interphase 10, the nuclear density amounts to about 10 nuclei per $250 \mu \mathrm{m}^{2}$. During interphase 11 the density of nuclei has doubled to 20 nuclei per $250 \mu \mathrm{m}^{2}$. Division of mother nuclei into two corresponding daughter nuclei continues also in the next interphase, such that after interphase 12 there are 40 nuclei per $250 \mu \mathrm{m}^{2}$. In interphase 13 , there are 80 nuclei per $250 \mu \mathrm{m}^{2}$. In their syncytial stage, i.e. during division cycles 9 to 13 , cortex of Drosophila [5, 33, with (Fig. 2.2, a-c). For the tracking studies presented within this work, this developmental window is interesting due to its strongly correlated internuclear dynamics [5]. After interphase 14, actin and microtubule mediated interaction of nuclei in the 2D nuclear array in the cortex decreases due to furrow canal formation separating individual nuclei [5, 35] (Fig. 2.2, d).

In the following we will give a brief overview of the microscopic non-equilibrium force generating machinery which plays an important role during embryonic development within living Drosophila embryos.

\section{Kinesin-5 motor protein}

Proteins are biological functional polymers consisting of amino acids [43. Motor proteins like kinesin-5 are vital for intracellular transport processes [44]. From a physical point of view, kinesin motor proteins are molecular non-equilibrium systems, driven by local energy dissipation [6, 7, 45, 47]. During stepwise binding of each motor head to mictrotubule strands, adenosine triphosphate (ATP to adenosine diphosphate (ADP) by releasing inorganic phosphate. This local energy disspation leads to processed directed locomotion of kinesin- 5 molecules on microtubule strands 7. Kinesin-5 motor proteins are important players in mitotic spindle dynamics and organization [7, 45,55]. In M phase, mitotic spindle filaments are pushed apart by kinesin-5 molecules processing along interpolar microtubules (Fig. 2.4. B) [7, 44]. In this work, we are interested in the correlative behavior of kinesin- 5 molecules in Drosophila embryos expressing enhanced green fluorescent protein with kinesin-5HaloTag@ (His-EGFP/kin-5-Halo) on long time scales (i. e. over at least one nuclear division cycle). We explore to what extent we can apply the HaloTag ${ }^{\circledR}$ method for specific binding of individual kinesin-5 motor proteins to individual functionalized fluorescent CNTs in living Drosophila embryos in successive nuclear division stages. 

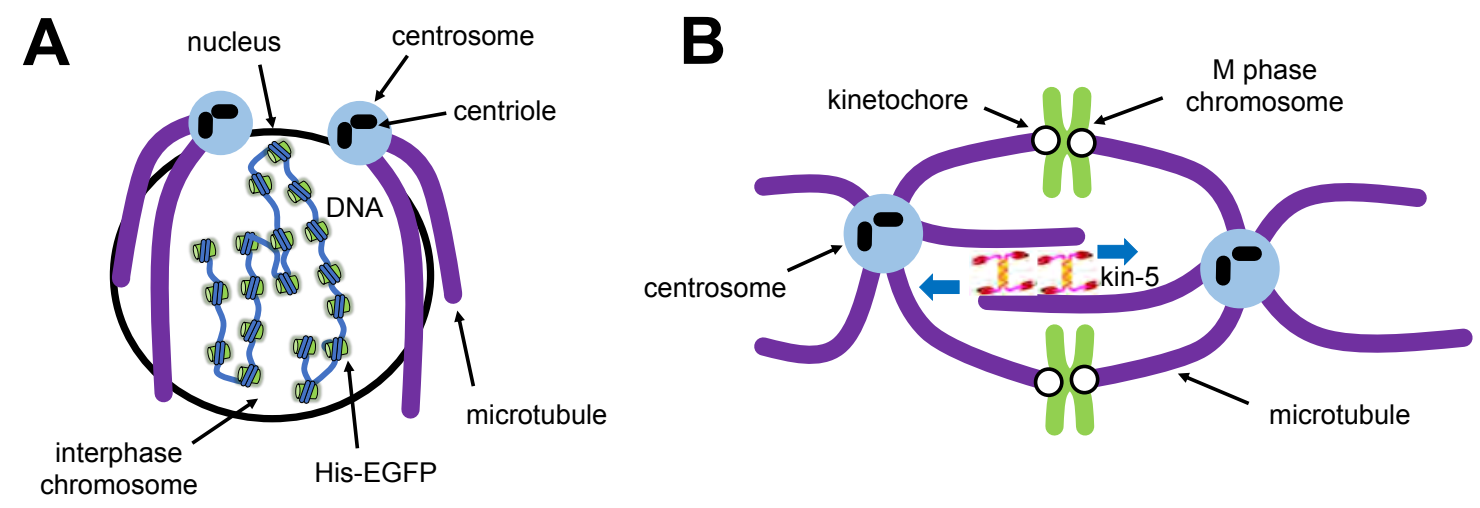

\section{C}

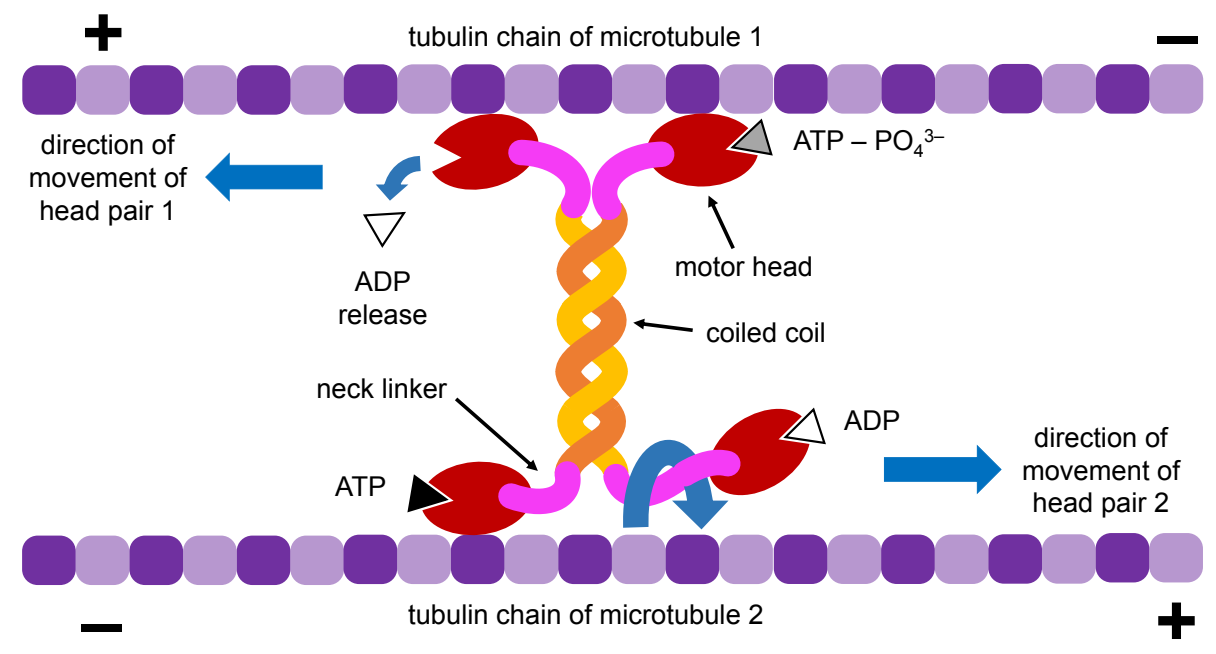

Figure 2.4. Schematic of the microscopic picture during cell division. A. Histone proteins enveloping DNA in a nucleus during interphase. B. Nucleus in M phase with kinesin-5 molecules sliding between spindle filaments 77, 44, 48, 50. C. Individual kinesin-5 tetramer with two head pairs (red) processing along microtubuli (violet) while locally dissipating ATP 7, 48. Each motor head contains a binding site for ATP consumption, leaving behind ADP after phosphorylation [7, typically towards "+" end [4]. The neck linker domain (magenta) connects the coiled-coil stalk (yellow and orange) with each motor head [7. 53, 56, allowing kinesin-5 motor heads to perform typical hand-over-hand movement 7]. 


\section{Cytoplasmic flow in Drosophila embryos}

The cytoplasm lies within the plasma membrane and embeds the nuclear array of Drosophila embryos [43, 57]. The cytoplasm comprises the watery cytosol, the cytoskeleton, organelles and proteins forming a crowded environment [43, [58, 59]. While the cortex is generally agreed to be primarily elastic [60], there is still controversy over whether the cytoplasm is best described as a weak elastic solid 61] or if the cytoplasm has viscoelastic structure [62. A complete understanding of embryonic morphogenesis demands comprehensive knowledge about the micromechanical structure of tissue [5, 60]. However, material properties in Drosophila embryos is still poorly understood in large parts 60. Occupying a large part of Drosophila embryos and inherently driven out of equilibrium, the cytoplasm provides an ideal system for studying active dynamics in vivo [22, 58, 63. Detection of non-equilibrium dynamics in the cytoplasm of Drosophila embryos requires understanding of the force generation machinery on microscopic level.

During embryonic development, the nuclear array in the cortex undergoes complex morphological rearrangement processes [64]. It is not entirely certain 64 what drives these rearrangement processes. Since the cortex is embedded in the cytoplasm, it is reasonable to search in the cytoplasm for contributions to the nuclear rearrangement process in the cortex. Biological systems are undeniably in thermodynamic non-equilibrium [7]. However, only little is known about possible non-equilibrium contributions to the control of cytoplasmic flow [37, 63. Neither are the driving forces and biological function of the cytoplasmic flow known [59. In this work, we present a promising method which, using CNTs and a non-invasive imaging method ( $\mathrm{PIV})$, can help to improve this knowledge by defining appropriate dynamic order parameters.

Due to their small size, CNTs are ideally suited to penetrate areas where conventional probes such as beads cannot reach [6] and thus decipher previously hidden microscopic mechanisms.

By means of PIV, kinesin activity and cytoskeletal organization have been found to correlate with cytoplasmic flow in Drosophila oocytes [63, 65]. In this work, we prove that PIV] can also be performed using CNTs as single-molecular tracer particles for imaging bulk dynamics in Drosophila embryos. 


\subsection{Carbon nanotubes}

\subsubsection{Carbon nanotubes as fluorescent probes in living organisms}

In this work, we study optical and dynamical properties of CNTs in Drosophila embryos. Since their discovery in 1991 [66] CNTs have widely aroused attention in physical and life sciences. CNTs display NIR fluorescence emission when subjected to particular laser light [20, 67, 68]. single-nanoparticle experiments in living organisms often use mesoscopic probes like beads with diameters ranging between hundreds of nanometers and even $1 \mu \mathrm{m}$ [5, 69, 70]. Individual single-walled CNT molecules can measure several hundred nanometers in length [6, 67, 171] but are only few nanometers in diameter [72 75]. Experiments have proven fluorescent CNTs to be ideal fluorescent stealth probes for studying microscopic dynamics in biological and other nonequilibrium systems [1, 6]. Incorporation of CNTs in living cells is minimally invasive and provides opportunities for targeted molecular sensing applications [76, 177] and protein specific binding [6]. The high potential of molecular NIR emitters in biomedical imaging has already been recognized [78 85]. While conventional fluorophores such as EGFP yield absorption-emission peaks being only some tens of nanometers apart [14], CNTs display Stokes shifts of up to several hundred nanometers [86-88] (Fig. 2.5 and Fig. 2.6).

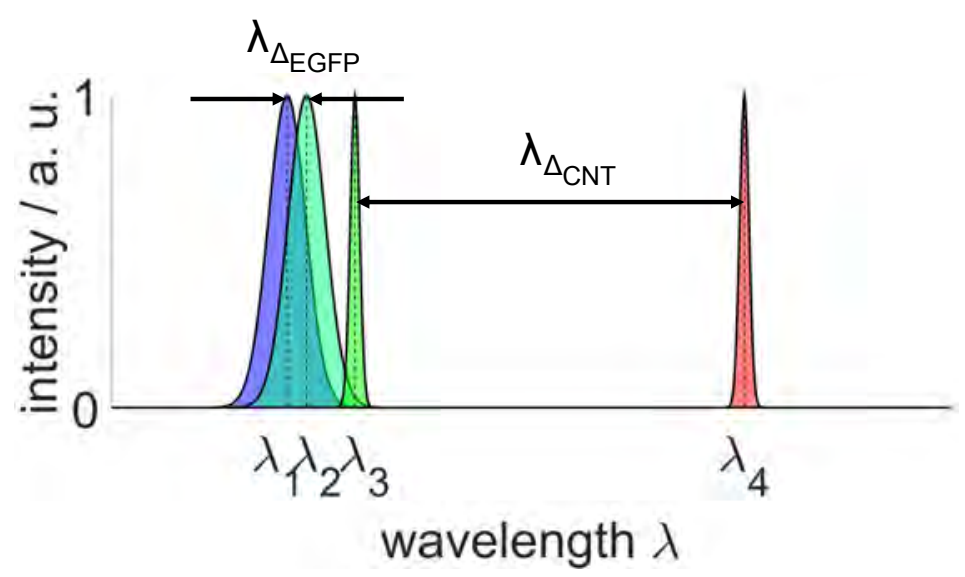

Figure 2.5. Schematic comparing Stokes shifts of CNTs and EGFP The Stokes shift of fluorescent CNTs can span up to several hundred nanometers $\left(\lambda_{\Delta_{\mathrm{EGFP}}}:=\lambda_{2}-\lambda_{1}\right)$, while commonly used fluorescent dyes, such as EGFP show Stokes shifts spanning only tens of nanometers $\left(\lambda_{\Delta_{\mathrm{CNT}}}:=\lambda_{4}-\lambda_{3}\right)$, i. e. $\left(\lambda_{\Delta_{\mathrm{EGFP}}} \ll \lambda_{\Delta_{\mathrm{CNT}}}\right)$. Here, $\lambda_{1}=488 \mathrm{~nm}$ and $\lambda_{1}=561 \mathrm{~nm}$ correspond to laser wavelengths being used for exciting fluorescence in EGFP and CNTs Corresponding emission wavelengths are at $\lambda_{2}=509 \mathrm{~nm}[16$ ] and $\lambda_{1}=976 \mathrm{~nm}$ [74. In these examples, Stokes shifts are $\lambda_{\Delta_{\mathrm{EGFP}}} \approx 20 \mathrm{~nm}$ for EGFP and $\lambda_{\Delta_{\mathrm{CNT}}} \approx 400 \mathrm{~nm}$ for CNTs with chirality $(n, m)=(6,5)$ Absorption wavelength $\lambda_{1}=561 \mathrm{~nm}$ and emission wavelengths $\lambda_{2}=976 \mathrm{~nm}$ are typical for CNTs with chirality $(n, m)=(6,5)$. Additionally, CNTs show a sharply defined spectrum. 
CNTs fluoresce beyond the VIS spectrum, leading to reduced background from the biological specimen, by circumventing autofluorescence [6, 89, 90] (Fig. 2.6).

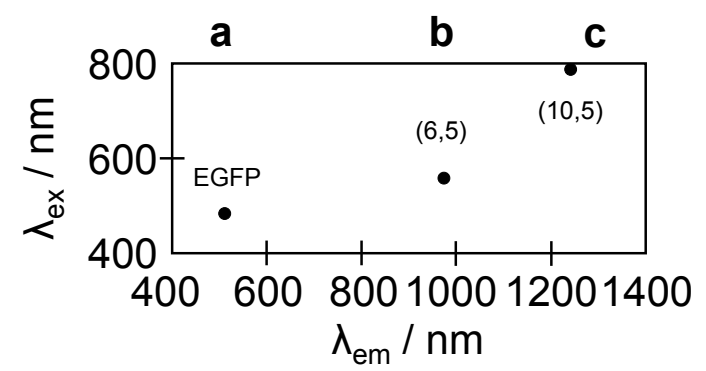

Figure 2.6. Schematic comparison of absorption-emission spectra of CNTs and EGFP EGFP is excited by $489 \mathrm{~nm}$ [14] and emits VIS fluorescent light at $509 \mathrm{~nm}$ [14, 16, (a). A CNT with chiral index doublet $(n, m)=(6,5)$ absorbs light in the VIS spectrum at $566 \mathrm{~nm}$ and emits fluorescence in the NIR at $976 \mathrm{~nm}$ [74 (b). CNTs with chirality $(10,5)$ show absorption in the NIR spectrum at $788 \mathrm{~nm}$ and emit fluorescence at $1249 \mathrm{~nm} \mathrm{[67]}$ (c).

Another huge advantage of NIR flouorophores over emitters with VIS wavelength is the reduced scattering of NIR light in biological specimens enabling deep imaging in tissues [78, 80, 82]. Additionally, it has been found that isolated fluorescent CNTs have a fluorescence lifetime in the order of 100 ps [91]. For more vigorous sonication of CNT suspensions, radiative lifetimes have been reported to be about 100 ns [92]. The lack of fluorescence intermittency in CNTs [18, 68, 93 95 and the fact, that photobleaching is suppresed in CNTs [6, 96] make CNTs stable photon emitters [18, 68, 94, 97, 98] and enable high temporal resolution of the dynamics on large observation time scales [6, 8]. In this work, we detect dynamics of CNTs in living Drosophila embryos for $30 \mathrm{~min}$ while continuously sampling at $10 \mathrm{~Hz}$. 


\subsubsection{Carbon nanotube geometry and fluorescence}

\section{Geometry}

CNTs are a carbonic allotropes with tube-shaped real-space configuration [20]. Their tube-shaped crystal structure appears as rolled-up graphene sheets. Like other modifications of carbon, CNTs obey very particular electronic properties. According to their optoelectronic properties, we can discriminate metallic and semiconducting CNTs [75, 99 102]. By means of $m, n, q \in \mathbb{N}$, we can quantify these to different classes of CNTs in terms of their chirality [67, 101, 103, 104]:

$$
\begin{array}{llll}
2 n+m=3 q & \Leftrightarrow & (n-m) \bmod 3=0 & \text { metallic } \\
2 n+m=1 \text { or } 2 & \Leftrightarrow & (n-m) \bmod 3=1 \text { or } 2 & \text { semiconducting . }
\end{array}
$$

The crystal structure of CNTs is determined by the definition of the basis vectors of the Wigner-Seitz cell in terms of the coefficients $(n, m)$. The primitive translation vectors $\mathbf{a}$ and $\mathbf{b}$ define the unit cell $\mathbf{c}$ of the CNT honeycomb lattice [101, 105]:

$$
\mathbf{c}=n \mathbf{a}+m \mathbf{b} \quad \text { with } m, n \in \mathbb{N}_{0} \text { and } \mathbf{a}, \mathbf{b} \in \mathbb{R}^{2} .
$$

The hexagonal lattice of a graphene sheet consists of carbon atoms distributed alterningly on two sub-lattices (Fig. 2.7, black and empty dots). Basis vectors a and $\mathbf{b}$ span the rhomboidal Wigner-Seitz cell of the graphene lattice. The linear combination of the basis vectors (see definition 2.2), determines the internal orientation of the CNT and thus also the optoelectronic behavior of the respective CNT as a whole. Differences in the optoelectronic properties are defined by the set of non-negative whole-numbered coefficients $(n, m)$ with $m, n \in \mathbb{N}_{0}$. It is equivalent to formulate the chiral index tupel $(n, m)$ in terms of the more illustrative chiral angle [101] (Fig. 2.7. dashed lines):

$$
\theta:=\tan \left(\frac{m}{2 n+m} \sqrt{3}\right) .
$$

Thus, there are three possible cases to construct optoelectronically distinct CNT lattice projections on graphene:

$$
\theta\left\{\begin{array}{lcc}
= & 0 & \text { "zigzag" } \\
= & \pi / 6 & \text { "armchair" } \\
\in(0, \pi / 6) & \text { "chiral" }
\end{array}\right.
$$

Figure 2.7 depicts the three possible cases defined in (Eq. 2.4): $\mathbf{c}_{\text {armchair }}=n(\mathbf{a}+\mathbf{b})$, $\mathbf{c}_{\text {zigzag }}=n \mathbf{a}$ and $\mathbf{c}_{\text {chiral }}=n \mathbf{a}+m \mathbf{b}$ with $\{n, m \in \mathbb{N} \backslash\{$ "zigzag" $\} \mid 2 n+m=1$ or 2$\}$ [67, 103, 104. 


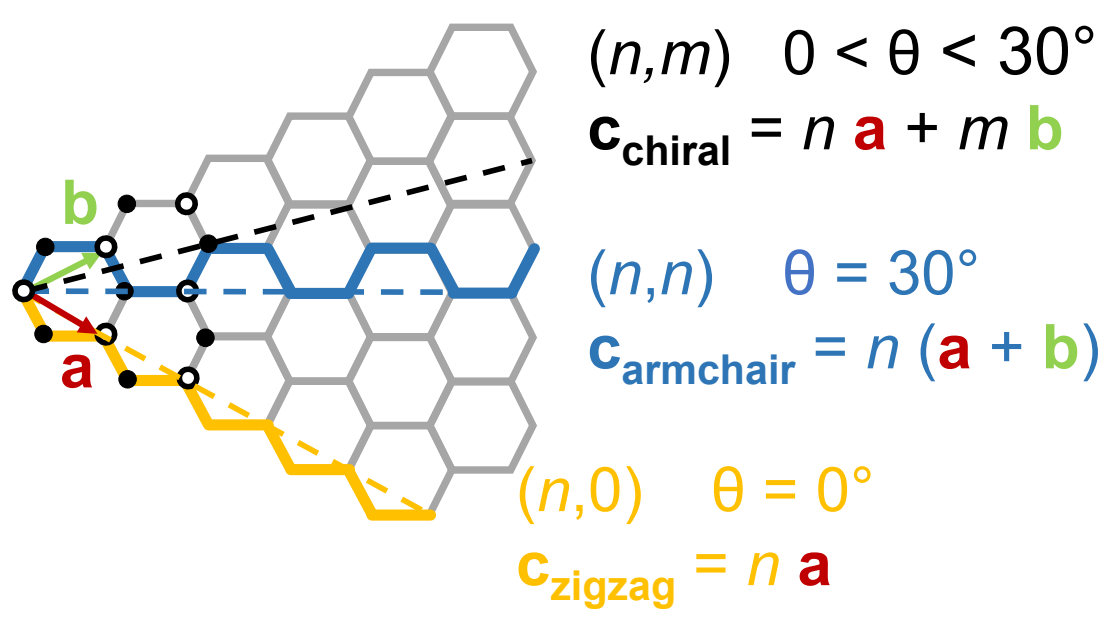

Figure 2.7. Schematic of a hexagonal lattice of a graphene sheet. A graphene sheet consists of carbon atoms belonging to two sub-lattices (black and empty dots) which form a monolayer in honeycomb structure. Vectors a (red) and $\mathbf{b}$ (green) span the Wigner-Seitz cell. Carbon atoms in a graphene sheet interconnect via $\sigma$ bonds originating from $s p^{2}$ hybridization [106] (light gray lines). Here, three optoelectronically different CNT types are depicted as projections on a graphene lattice: "zigzag" $(n, 0) \Leftrightarrow \theta=0$ (yellow) and "armchair" $(n, n) \Leftrightarrow \theta=\pi / 6$ (blue) CNTs and CNTs of the general "chiral" type $(n, m)$ with $\{n, m \in \mathbb{N} \backslash\{$ "zigzag" $\} \mid 2 n+m=1$ or 2$\} \Leftrightarrow 0<\theta<\pi / 6$ [67, 103, 104] type (black) [3, 88, 103]. 


\section{Carbon nanotube fluorescence}

Fluorescence is a form of luminescence, that is, the spontaneous emission of light in a quantum process. During fluorescence, a fluorophore is excited at a wavelength $\lambda_{\text {ex }}$. Due to this excitation, a photon of higher wavelength $\lambda_{\mathrm{em}}$ is emitted. We define the difference between these wavelengths as $\lambda_{\Delta}:=\lambda_{\mathrm{em}}-\lambda_{\mathrm{ex}}$ and call it Stokes shift (Fig. 2.8 a and c).

Fluorescence is a non-classical phenomenon based on transition of electrons in singulet states. Singulet states $S_{0}$ and $S_{1}$ can be approximated each by a Morse-like potential [107, 108]

$$
V(r)=D_{\text {eq }}\left(1-e^{A \cdot\left(r-r_{\mathrm{eq}}\right)}\right)^{2},
$$

accounting for the convergence of higher vibrational states [107]. In (Eq. 2.5), $r_{\text {eq }}$ is the equilibrium position of the anharmonic oscillator. Amplitude of the anharmonic potential $V(r)$ is defined by the dissociation energy $D_{\text {eq }}$ which also defines and potential stiffness $A=\sqrt{k_{\text {eq }} / 2 D_{\text {eq }}}$ depending on $D_{\text {eq }}$ and spring constant $k_{\text {eq }}$.

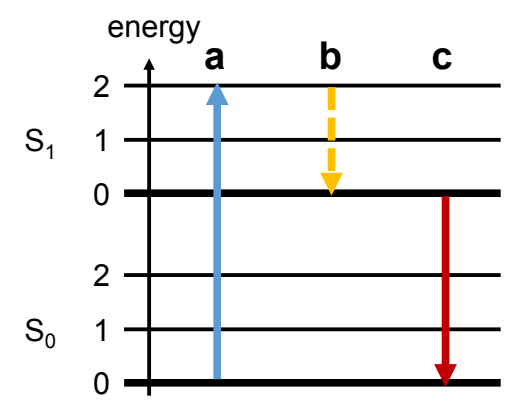

Figure 2.8. Schematic of a fluorescence transition. A fluorescence transition can occur between two singulet states $S_{0}$ and $S_{1}$ with ground energy $E_{0}=0$. Fluorescence excitation from an energetically lower singulet state $S_{0}$ to an energetically higher singulet state $S_{1}$ (a). Non-radiative internal vibrational transition inside the energetically higher singulet state $S_{1}$ down to its ground state $(E=0)(\mathbf{b})$. Actual fluorescence transition of an electron leaping from the ground state of the energetically higher singulet state $S_{1}$ to an excited state of an energetically lower singulet state $S_{0}(\mathbf{c})$.

Semiconducting CNTs are single-nanoparticle emitters of NIR light [20, 88, 91, 109]. Fluorescence is mediated between band gaps in the order of $1 \mathrm{eV}$ [74, 110, 111]. Band gap fluorescence of CNTs [17, 111] displays narrow peaks on a wide-range excitation-emission spectrum [17, 18, 68, 88]. Fluorescence transitions in CNTs occur between Van Hove sigularities (Fig. 2.9). Formation and recombination of excitons (electron-hole pairs) in semiconducting CNTs is inevitable to elicit fluorescence in semiconducting CNTs [19, 95, 103, 104, 111-118]. Fluorescent CNTs are molecular photon sources with a quantum yield of $10 \%$ [18, 67, 94, 119]. Geometric parameters of CNTs, such as tube diameter or crystal structure as well as defects contribute enormously to the optoelectronic properties of CNTs [67, 74, 120. CNTs with $(10,5)$-chirality have been found to absorb photons at $\lambda_{\text {abs }}=788 \mathrm{~nm}$ and an emission wavelength of $\lambda_{\mathrm{em}}=1249 \mathrm{~nm}$ [67] (Fig. 2.6). In our work, we use CNTs with fluorescence emission peaks at $561 \mathrm{~nm}$ and $770 \mathrm{~nm}$. From our measurements, we assume our batch of CNTs to contain a considerable amout of CNTs with $(10,5)$ - 
chirality. CNT fluorescence is not only influeced by CNT geometry but also by the chemical environment CNTs are embedded in. For instance, rearrangement of charge distribution due to the wrapping agent and the choice of solvent can strongly influence CNT fluorescence [121 132]. In the presence of metallic CNTs intertube van der Waals interactions, arising from intertube $\pi-\pi$ interactions of nonhybridized $2 p$ orbitals in carbon [106, 133], can quench fluorescence in semiconducting CNTs 20, 94, 113, 134. In this work, we have used CNTs with excitation maxima at $561 \mathrm{~nm}$ and $770 \mathrm{~nm}$. Figure 2.6 illustrates schematically absorption-emission spectra of two species of functionalized CNTs emitting NIR fluorescent light. In this work, we present a systematic investigation of the optical properties of individual fluorescent CNT molecules in living Drosophila embryos, bequeathing information about the point spread function $(\mathrm{PSF})$. This work also provides insights into the dynamical behavior of fluorescent CNTs molecules in Drosophila embryos for singlenanoparticle experiments during globally recumbent nuclear motion and for bulk motion of cytoplasmic streaming.

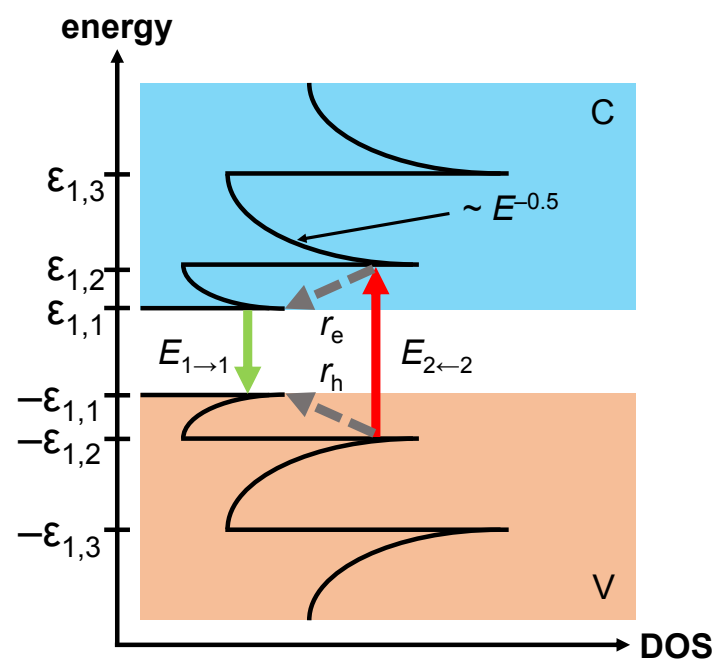

Figure 2.9. Schematic of the fluorescence transition in a semiconducting CNT 88, 135]. Horizontal lines symbolize the Van Hove singularities in the electronic DOS In this example, fluorescence emission appears as an inter-band transition $E_{1 \rightarrow 1}:=E_{c_{1}}-E_{v_{1}}\left[75\right.$ after photon absorption from the respective $E_{2}$ states in conduction band $(\mathrm{C})$ and valence band $(\mathrm{V})$, with the respective Van Hove singularities in the density of states (DOS) defining the energy transition edges. Dashed gray arrows symbolize intra-band non-radiative relaxations between electrons $r_{\mathrm{e}}$ and holes $r_{\mathrm{h}}$.

The fluorescence transition in CNTs occurs between the first excited energy states $E_{1}$ of the conduction band and the valence band. To understand the origin of fluorescence in CNTs, we first have to understand the role of Van Hove singularities [116, 117, 136], the springpoint of CNT fluorescence. It is suitable to regard an isolated CNT as an electron system, confined to one dimension. In such a "particle-in-a-box" quantum system, the corresponding stationary Schrödinger equation reads

$$
\hat{H}\left(\varphi_{n_{y}, n_{z}}(y, z) \cdot \exp \left(i k_{x} x\right)\right)=E_{n_{y}, n_{z}} \varphi_{n_{y}, n_{z}}(y, z) \cdot \exp \left(i k_{x} x\right) .
$$

From equation (Eq. 2.6) it is straight forward, to find energy eigenvalues 


$$
E_{n_{y}, n_{z}}=\frac{\hbar^{2} k_{x}^{2}}{2 m}+\varepsilon_{n_{y}, n_{z}}
$$

with $\varepsilon_{n_{j}} \simeq n_{j} / L_{j}$ [135]. Using periodic boundary conditions, wave vector components can take values $k_{n_{j}}=2 \pi n_{j} / L_{j}$, with $L_{j}$ being the potential well width in $\hat{H}$ (Eq. 2.7). In this case, $n_{y}$ and $n_{z}$ are $(2 k+1)$-fold degenerate $(k \in \mathbb{N})$. We introduce DOS $g(\varepsilon)$ as the total number of all states $N$ occupying an energy interval $\varepsilon^{\prime} \in \hbar[\omega(\mathbf{k}) ; \omega(\mathbf{k})+\Delta \omega]$ :

$$
g(\varepsilon)=\frac{d N}{d \varepsilon} .
$$

For a cubic $k$-space volume element $k=(2 \pi / L)$, one state in a one-dimensional (1D) $k$-space occupies a volume of

$$
V_{k}^{\text {unit }}=\frac{2 \pi}{L} .
$$

For a 1D system like CNTs, the total $k$-space volume of the system reduces to a line element:

$$
V_{k}^{\mathrm{tot}}=k .
$$

The total number of states $N_{k}^{\text {tot }}$ in the total $k$-space volume $V_{k}^{\text {tot }}$ can then be defined by the ratio of (Eq. 2.10) and (Eq. 2.9):

$$
N \equiv N_{k}^{\text {tot }}=\frac{V_{k}^{\text {tot }}}{V_{k}^{\text {unit }}}
$$

Using (Eq.2.11) and the quadratic dispersion relation (Eq.2.7), a root-like divergence appears for the 1D DOS,

$$
g(E)=\frac{d N}{d \varepsilon}=\frac{\partial N}{\partial k} \frac{d k}{d \varepsilon} \propto \frac{1}{\sqrt{\varepsilon}} .
$$

Under consideration of quantum numbers $n_{y}$ and $n_{z}$, we obtain a total DOS by summation of the subbands:

$$
\begin{aligned}
g_{\mathrm{tot}}(\varepsilon) & =\sum_{n_{y}, n_{z}} g_{n_{y}, n_{z}}(\varepsilon) \\
& \sim \sum_{n_{y}, n_{z}} \frac{1}{\sqrt{E-\varepsilon_{n_{y}, n_{z}}}}
\end{aligned}
$$

elsewise, $g(\varepsilon)=0$ [135]. The result of (Eq. 2.13) delivers the overall DOS, dependent on sub-band quantum numbers $n_{y}$ and $n_{z}$. 



\section{Materials and sample preparation}

In this chapter, we display the incorporation of watery CNT suspensions into Drosophila embryos by microinjection. 1 Protocols of Fakhri et al. [6] have served as basis for CNT supsension preparations and subequent CNT functionalization. The author thanks Dr. Kengo Nishi for preparation of functionalized CNTs which have been used for the on hand in vivo experiments with Drosophila.

\subsection{Drosophila constructs}

For the studies presented in this work, we have used two transgenic fly crosses:

- His-EGFP and

- His-EGFP/kin-5-Halo.

Here, we compare these two species to each other with respect to the dynamical behavior of NIR fluorescent CNTs inside the cortical layer of the respective Drosophila embryo. We also apply CNTs as fluorescent markers to passively probe bulk motion of embryonic cytoplasm during late interphase by means of PIV (Chap. 6.4). In all our experiments (Chap. 5.2, 6.3.1, 6.4) we use embryos expressing His-EGFP. By this we obtain spatio-temporal orientation (Chap. 6.1.2). By labeling histones with EGFP, we directly observe nuclear morphology and thus have at each timepoint an exact knowledge about the developmental stage and the thermodynamic fate of the embryo. Additionally, we obtain spatial information, since the EGFP is expressed in the nuclei which we observe in the cortical region between interphases 10 to 13 . Spatial orientation is indispensable in our studies, since EGFP-tagged histones allow clear and exact orientation of the focal plane inside the embryo which is vital to find the correct image plane for experiments with CNTs in the cortex (Chap. 6.3). For assessing efficiency of applying CNTs as markers for labeling individual kinesin-5 molecules we have used embryos expressing His-EGFP/kin-5-Halo, This means that flies have been modified to express kinesin-5 motor proteins with a HaloTag ${ }^{\circledR}$ [137] binding site to potentially bind to individual CNTs via a succinimidyl ester (SSE) (HaloTag ${ }^{\circledR}$ Succinimidyl Ester (O4) Ligand; Promega GmbH, Mannheim, Germany) based ligand (Fig. 3.1).

\footnotetext{
${ }^{1}$ Fly culture is based on standard protocols as applied in the lab of Prof. Dr. Jörg Großhans.
} 


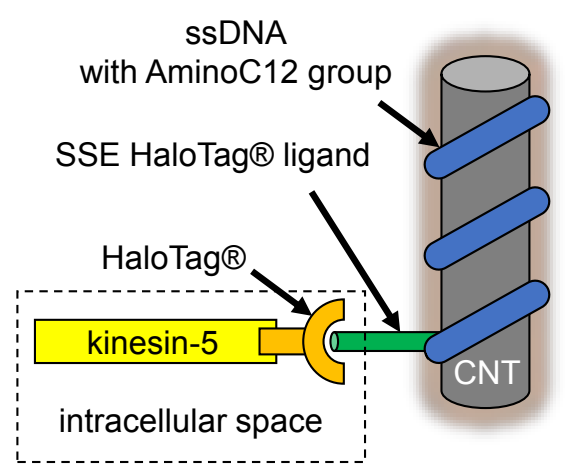

Figure 3.1. Schematic of CNT functionalization. The CNT (gray) is wrapped in the ssDNA (blue). During the functionalization process, the functional amino group at the $\mathrm{dT}_{3} 0$ SsDNA obtains the SSE (green). The SSE serves as a crosslinker between the CNT wrapped in SSDNA and the kinesin-5 motor protein (yellow) with its HaloTag ${ }^{\circledR}$ (orange). This part is incorporated into the Drosophila embryo via microinjection. The part of the illustration inside the dashed box, the kinesin-5 protein with the HaloTag ${ }^{\circledR}$, are expressed inside the embryo.

In this work, we investigate, to what extend $\mathrm{CNTs}$ functionalized with a HaloTag ${ }^{\circledR}$ ligand can bind to individual kinesin-5 molecules in His-EGFP/kin-5-Halo embryos. As a control, we used embryos without a HaloTag ${ }^{\mathrm{R}}$ binding site at kinesin-5. The control embryos are modified to express His-EGFP. One central goal of this work is to investigate and compare the dynamic behavior of functionalized CNTs in embryos of these two transgenic fly crosses (Chap. 6.3).

\subsection{Preparing and functionalization of carbon nanotubes}

In this chapter we present a method for suspending hydrophobic CNTs in watery solutions using biocompatible polymers such as ssDNA, To disperse CNTs we apply probe tip sonication. We use CNTs which are wrapped in SsDNA with a functional AminoC12 group at the $5^{\prime}$ end. During functionalization this AminonC12 group potentionally covelently binds to a HaloTag ${ }^{\circledR}$ SSE ligand which in turn potentially binds to HaloTag ${ }^{\circledR}$ modified kinesin-5 in His-EGFP/kin-5-Halo Drosophila embryos. For CNT suspension and functionalization, we here follow the protocol presented in [6].

\section{Statement}

Both Dr. Kengo Nishi and Constantin Kohl performed and optimized the CNT suspensions. The protocol for CNT functionalization is reported by Dr. Kengo Nishi. The CNTs for injection into Drosophila embryos, which led to the results in chapters (Chap. 6.3) were prepared by Dr. Kengo Nishi. Here, we present the preparation process of the CNT samples, which were also used in experiments for fluorescence imaging with living Drosophila embryos. 


\subsubsection{Suspending carbon nanotubes in watery environments}

\section{Weighing dry carbon nanotubes}

The first step in CNT preparation is the weighing of dry CNTs, Weighing CNTs has turned out to be a challenging task, since they display strong electrostatic affinity and have a low density. This became particularly noticeable during the transferring of dry CNTs despite grounding and using antistatic spatulas (VWR Polypropylene Spatula Eco; VWR ${ }^{\circledR}$ International GmbH, Dresden Germany).

We used a micro balance (CP224S; Sartorius AG, Göttingen, Germany) for weighing $1 \mathrm{mg}$ dry CNTs. However, the resulting masses of dry CNTs (HiPCO single-walled carbon nanotubes, batch number 189.2; Rice University, Houston, Texas, USA) were subject to large fluctuations and it turned out that determining a nominal CNT mass in the range of $1 \mathrm{mg}$ required a high degree of practice and experience in handling the material used.

In the following, we present a another mass independent approach to determine the amount of CNTs used during experiments which initially seemed attractive since by this we could circumvent large mass fluctuations (about $10 \%$ of the nominal mass). We determined the volume of the sample by measuring the diameter of the glass vial $(4 \mathrm{ml}, 14.75 \times 45 \mathrm{~mm}$, Vials N13 flat Screw Neck 13-425; MACHEREY-NAGEL GmbH \& Co. KG, Düren, Germany) and the height $h_{\mathrm{CNT}}$ of CNT sample. Assuming the dry CNTs to be confined to a cylindrical volume, we can calculate the volume $V_{\mathrm{CNT}}$ of dry CNTs using the inner circular basal area $G_{\text {vial }} \approx 127.68 \mathrm{~mm}^{2}$ of the cylindrical vial and the height $h_{\mathrm{CNT}} \approx 15 \mathrm{~mm}$ of the CNT heap inside. This results in a CNT volume of

$$
V_{\mathrm{CNT}}=G_{\mathrm{vial}} \cdot h_{\mathrm{CNT}} \approx 1.9 \mathrm{ml}
$$

In contrast to directly weighing CNTs which required experience in handling dry CNTs, a volume based approach (Eq. 3.1) for determining the amount of CNTs used in the further course of experiments allowed us to obtain a reproducible amount of CNTs. However, this volume based approach unfortunately resulted in more inhomogeneous dispersion state of CNT suspensions, making this approach less efficient than a direct mass determination of dry CNTs. As we found later, it was inevitable to lose CNTs during later sonication and transferring of CNT suspensions. During imaging we found that the initial weighing precision of the dry CNTs is less critical to obtain comparable results in the dispersion state.

\section{Wrapping carbon nanotubes with a biocompatible charged phase}

To ensure compatibility with the watery environment within embryos we have to overcome the hydrophobic properties of the unpolar CNTs during in vitro preparation of CNT suspensions by surrounding CNTs with a hydrophilic phase [138, 139]. In this work, we wrap CNTs in ssDNA to ensure biocompatibility [140]. By wrapping the CNTs with ssDNA, the base pairs of the ssDNA interact via $\pi-\pi$ interactions with the CNTs [141], the backbone of the ssDNA (composed of phosphate and desoxyribose), which in turn allows hydrophilic interaction of the enclosed CNT, causing the CNTs to suspend in water [139 141]. 
For CNT wrapping we use ssDNA with 30 thymine bases ( $\mathrm{dT}_{30} 30$-mer), with an AminoC12 group at its $5^{\prime}$ end (dT30 AminoC12, Thermo Fisher Scientific Life Technologies GmbH, Darmstadt, Germany), because comparisons between ssDNA and different base sequences showed that the fluorescence yield is highest at $\mathrm{dT}_{30}$ ssDNA [139. We dissolve $2 \mathrm{mg}$ of the 30-mer in $2 \mathrm{ml} 0.1 \mathrm{M} \mathrm{NaCl}$ solution in a glass scintillation vial $(4 \mathrm{ml}, 14.75 \times 45 \mathrm{~mm}$, Vials N13 flat Screw Neck 13-425; MACHEREY-NAGEL GmbH \& Co. KG, Düren, Germany). We then store the sample in an ice-water bath for further processing.

\section{Sonicating carbon nanotubes in watery suspensions}

After dissolving SsDNA in watery $\mathrm{NaCl}$ solution, we add CNTs, we use probe sonication (VC $5020 \mathrm{kHz}, 50 \mathrm{~W}$, SONICS \& MATERIALS INC., Danbury, CT, USA) (Fig. 3.2) to disperse aggregates and thus to assure an optimal dispersion state of CNT suspensions. Sonication breaks inter-tube van der Waals bonds in CNTs [142] resulting in homogeneously dispersed suspensions.

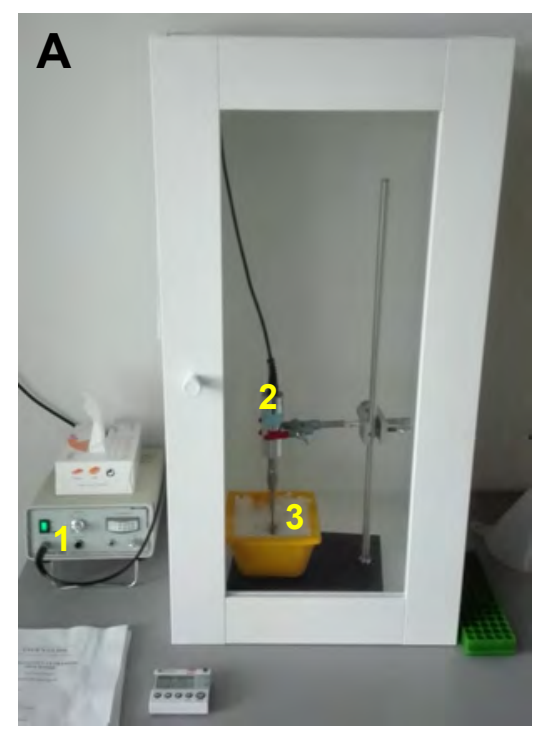

B
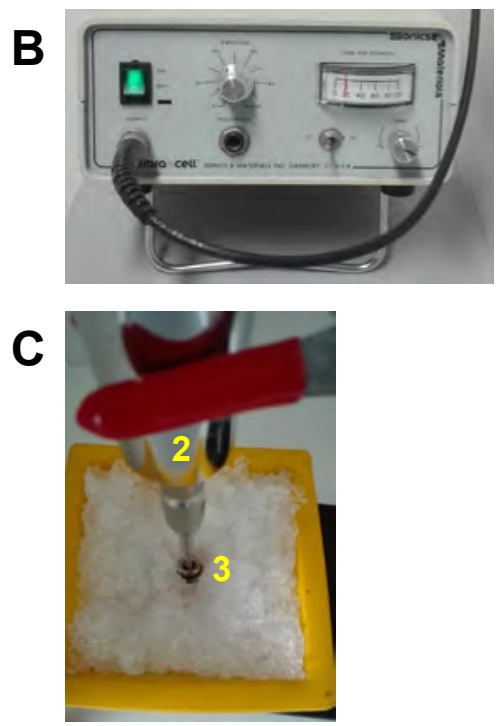

Figure 3.2. Sonication setup. A. Probe sonicator setup comprising power control (1), sonication microprobe tip (2) and ice filled bucket (3) with a vial containing $1 \mathrm{mg}$ CNTs and $\mathrm{dT}_{30}$-SsDNA dissolved in water. B. Zoom into the control unit of the sonicator. C. Zoom into the setup showing the microprobe tip (2) sonicating CNT suspension in a glass scintillation vial, embedded in a heat sink consisting of a bucket filled with ice and water (3).

Based on previous experience with the materials used and the sonication setup [6], we sonicate the sample for $t_{\mathrm{s}}=90 \mathrm{~min}$ at a power of $P_{\mathrm{s}}=10 \mathrm{~W}$ with a $2 \mathrm{~mm}$ microprobe tip (Fig. 3.2). During the sonication procedure, we have access to four parameters: sonication power $P_{\mathrm{s}}$, sonication time $t_{\mathrm{s}}$, sample volume $V_{\mathrm{s}}$ and probe tip depth. In studies with surfactant-wrapped CNTs, the degree of CNT dispersion has been characterized by the sonication energy $E[143]$ combining $P_{\mathrm{s}}, t_{\mathrm{s}}$ and $V_{\mathrm{s}}$ :

$$
E=\frac{P_{\mathrm{s}} \cdot t_{\mathrm{s}}}{V_{\mathrm{s}}}
$$


In our case, we obtained a value of $E=27 \mathrm{~kJ} / \mathrm{ml}$. To prevent thermal damage of the CNT suspension throughout sonication, we place the glass scintillation vial containing CNTs and the watery SsDNA solution in an water-ice bath [142. The water-ice mixture guarantees isothermal conditions at $0^{\circ} \mathrm{C}$ with reasonable accuracy. To not damage the sensitive membrane of the microprobe tip, it is important that it does not come into contact with anything but the suspension. Throughout the whole sonication process we ensured the microprobe tip to have a distance of about $2 \mathrm{~mm}$ to the bottom of the glass scintillation vial. During the sonication process we observed an incomplete dissolution of the CNTs. Furthermore, aggregates of wet CNT adhered macroscopically visible to the glass scintillation vial in an uncertain amount.

\section{Collecting the supernatant of carbon nanotube suspensions}

In order to ensure the observation of individual CNTs during in vivo fluorescence imaging, we centrifuged the suspension at 16,000 rpm for $60 \mathrm{~min}$ and carefully collected the supernatant which was then stored at $4^{\circ} \mathrm{C}$.

\subsubsection{Functionalizing carbon nanotubes}

In our studies, we used transgenic Drosophila, expressing kinesin-5 motor proteins with a HaloTag ${ }^{\circledR}$ binding site for potentially specifically targeting kinesin-5 motor proteins (His-EGFP/kin-5-Halo) with fluorescent CNTs [133].

We use [6] as the basis of this functionalization protocol.

A successful fusion of CNTs to kinesin-5 motor proteins requires functionalization of above-mentioned CNT suspensions. Here, we use a SSE based HaloTag ${ }^{\circledR}$ ligand (HaloTag ${ }^{\circledR}$ Succinimidyl Ester (O4) Ligand; Promega GmbH, Mannheim, Germany) potentially binding covalently to the AminoC 12 end of $\mathrm{dT}_{3} 0$-ssDNA. In the following, we will use the terms HaloTag ${ }^{\circledR}$ ligand and SSE synonymously.

The design of new synthetic exogenous fluorophores offers the advantage of being able to control their fluorescence properties almost entirely. However, their specific binding in living organisms is often complicated. The HaloTag ${ }^{\circledR}$ technology allows a specific coupling of externally incorporated fluorophores to specific molecules by genetically encoding protein specific binding sites for exogenous probes [133, 137]. Three building blocks are required in this process: the HaloTag ${ }^{\circledR}$ modified protein with a specific binding site, the externally introduced fluorophore, and thirdly a HaloTag $\left.^{(}\right)$ligand that can covalently bind exogenous fluorophore and the genetically modified protein [137.

In this section, we present a protocol for CNT functionalization to potential link them to kinesin-5 motor proteins in Drosophila embryos. We apply these functionalized CNT] ssDNA constructs to embryos expressing HaloTag $\left.{ }^{(}\right)$modified kinesin-5 proteins His-EGFP/kin-5-Halo). Using the same functionalized CNTs, we compare His-EGFP/kin-5-Halo embryos with a control without a HaloTag ${ }^{\circledR}$ binding site at kinesin-5 proteins (His-EGFP embryos) (Chap. 6.3).

At first, $100 \mu \mathrm{l}$ CNT]ssDNA suspension is filled in a $4 \mathrm{ml}$ molecular weight cutoff (MWCO $100 \mathrm{kDa}$ filter (Amicon ${ }^{\circledR}$ ) Ultra $4 \mathrm{ml}$ Centrifugal Filters Ulatracel ${ }^{\circledR}$ ) 100K; Merck, Molsheim, France) for filter centrifugation (Allegra ${ }^{\circledR}$ X-15R Centrifuge; BECKMANN COULTER, Brea, CA, USA). During centrifugation $10 \mu \mathrm{l}$ of 
$50 \mathrm{mg} / \mathrm{ml} \mathrm{HaloTag}^{\circledR}$ ) ligand (HaloTag ${ }^{\circledR}$ Succinimidyl Ester (O4) Ligand; Promega GmbH, Mannheim, Germany) are dissolved in dry dimethyl sulfoxide (DMSO) (Sigma-Aldrich Biochemie GmbH, Hamburg, Germany). After filtering, we add the HaloTag $^{\mathrm{R}}$ ligand dissolved in dry DMSO and add $11 \mu$ l phosphate-buffered saline $(10 \times$, Thermo Fisher Scientific Life Technologies GmbH, Darmstadt, Germany) to the CNT suspension to start the reaction between HaloTag ${ }^{\mathrm{R}}$ ligand and the AminoC12 group at the ssDNA. We incubate the vial for $2 \mathrm{~h}$ at room temperature. In this time the AminoC12 group at $\mathrm{dT}_{30}$ 30-mers can covalently bind to the SSE. By filtering the suspension three times (Centrifuge 5417 R; Eppendorf AG, Hamburg, Germany) with a MWCO $10 \mathrm{kDa}$ filter (Amicon ${ }^{\mathrm{R}}$ Ultra $0.5 \mathrm{ml}$ Centrifugal Filters Ulatracel $^{\circledR}$ 10K; Merck, Molsheim, France) excess HaloTag ${ }^{\circledR}$ ligand is removed.

\subsection{Preparation of Drosophila embryos}

In this work, we use microinjection to incorporate CNTs into Drosophila embryos. Experiments with fluorescent beads as tracer particles inside the Drosophila using microinjection have been performed by Weßel at al. [5]. In our work, we inject CNTs into Drosophila embryos. During this procedure, a glass needle of few micrometers in diameter containing CNT suspension is inserted into an Drosophila embryo releasing the liquid in the needle into the embryo. This procedure is schematically illustrated in figure 3.3. Before microinjecting the CNT suspension, Drosophila eggs have to be collected in a particular window of developmental stage. The collection process is illustrated in figure 3.5. During both the collection of eggs and the injection procedure of CNTs into Drosophila embryos, we use two stereo light microscopes (Fig. 3.4). With the first microscope (Fig. 3.4. A), we collect and align eggs. The second stereo microscope is used for the actual injection procedure (Fig. 3.4. B).

\subsubsection{Collection of Drosophila eggs}

Before we start the fluorescence imaging experiments with Drosophila embryos, we have to collect the eggs from their breeding ground and align them for microinjection with CNT suspension. During the time of experiments with Drosophila and CNTs, adult Drosophila flies live, reproduce and lay their eggs in a cylindrical cage with a lid. Air circulation inside the cage is ensured by a fine grid on one end of this tube. The other end of the tube is closed by a lid filled with hardened agar-apple juice (HiPP Bio-Saft Milder Apfel; HiPP Holding, Pfaffenhofen (Ilm), Germany and Agar Agar SERVA high-gel strength; SERVA Electrophoresis GmbH, Heidelberg, Germany), providing the flies with liquid. As a food source, we provide them baker's yeast (42.5 g, Backhefe DHW vital gold; Deutsche Hefewerke GmbH, Nuremberg, Germany) mixed with water (about 5 to $10 \mathrm{ml}$ ). We spread the yeast on the agar-apple juice plate coating it with a thin layer.

After the flies are placed into the tube, we seal the lid with tape and turn the whole cage on its lid (Fig. 3.3. A). Since Drosophila prefer a dark and warm environment, we keep the cage in a cabinet at standard conditions. The agar-applejuice-yeast plate also serves as egg deposition for the adult flies (Fig. 3.3, A). Since for our studies embryos at early stages are interesting, we exchange lids every $30 \mathrm{~min}$. In 
order to get rid of layers which could disturb during imaging, we remove the layer by subjecting the Drosophila eggs to a $50 \%$ hypochlorite solution (DanKlorix; CP GABA, Hamburg, Germany) for 2 min (Fig. 3.3. B). It is important to keep this time, in order to not harm the embryos. By stirring the hypochlorite solution with a fine paintbrush, we carefully remove embryos from the agar-apple juice plate (Fig. $3.3, \mathrm{~B})$.

After these $2 \mathrm{~min}$, we pour the content of the lid on a fine grid to collect the eggs (Fig. 3.3. C). Now, the eggs on the grid and have to be rinsed carefully under flowing water (Fig. 3.3, C).

With a clean fresh paintbrush we tansfer them from the grid (Fig. 3.3, D) on a fresh piece of gelatinous agar-apple juice (Fig. 3.3, E.a). With a fine sewing needle, we sort the embryos on the objective slide from the heap and lift them one by one to their right place (Fig. 3.3, E.b). This arrangement is important, since it will later be seen unchanged during the experiments. During this step, we use a stereo microscope with up to $4 \times$ magnification (Fig. 3.4. A). The lined up dechorionated embryos are glued (Tesa ${ }^{\circledR}$; Beiersdorf, Hamburg, Germany, dissolved in heptane) on a glass coverslip by flipping the glued side of the coverslip down and carefully placing it on the embryos (Fig. 3.3 , F). It is important to only exert as much force as needed to just make the embryos stick on the glue on the glass cover slip and not damaging them in the process.

We dry embryos in a box with hygroscopic beads for 10 to 15 min in order to remove excess water from the embryos (Fig. 3.3. G). After covering of Drosophila embryos with 10S oil (Voltalef 10S oil, Lehmann \& Voss, Hamburg, Germany), the actual microinjection procedure can start (Fig. 3.3, F). 
A

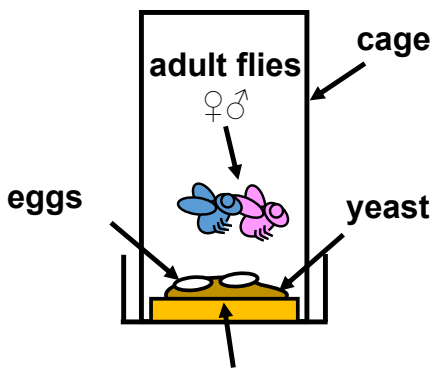

agar-apple juice plate

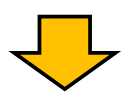

B
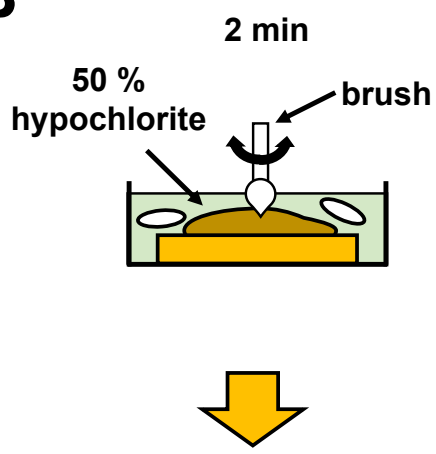

C
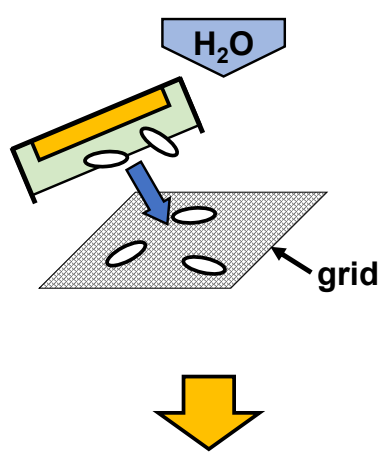

D

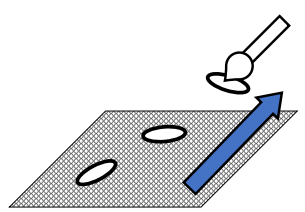

E

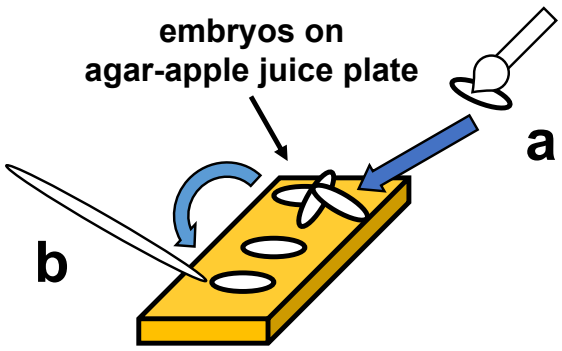

F

\section{glue on \\ glass coverslip \\ facing embryos}
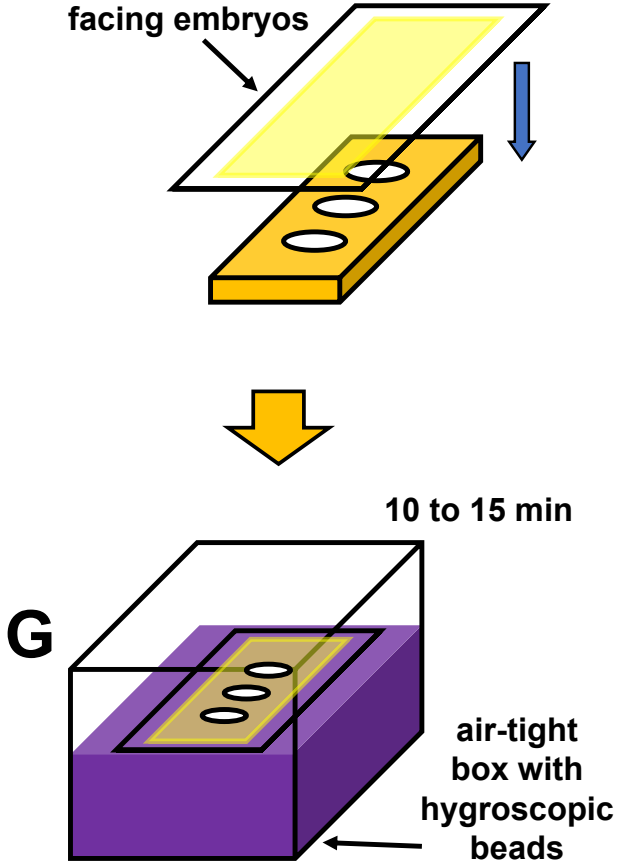

H
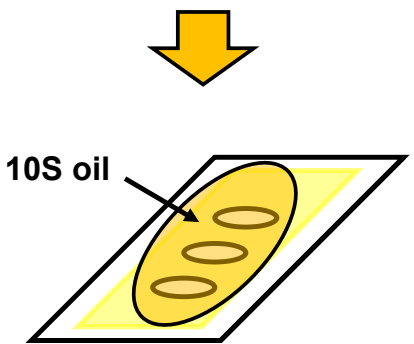

Figure 3.3. Schematic of sample preparation before microinjetion of CNT suspension. A. Adult Drosophila flies live and breed in a cylindrical cage. B. Drosophila eggs are exposed for 2 min to $50 \%$ Klorix solution for dechorionation. During the dechorionation process, embryos are detached carefully from the agar-apple juice-yeast plate with a brush. C. Eggs are transferred to a grid and rinsed under flowing water to remove excess Klorix solution. D. Dechorionated embryos are collected from the grid with a fine brush. E. Embryos are placed on a fresh agar-apple juice plate. F. Embryos are arranged one by one on a glue-coated glass coverslip by placing the glass coverslip with its glued site down carefully on the aligned embryos. G. Embryos are dried for 10 to 15 min. H. Embryos are covered with $10 \mathrm{~S}$ oil to prevent them from complete dehydration. Reproduced from "Near-infrared fluorescence imaging of single-walled carbon nanotubes in living organisms", which has been submitted to Review of Scientific Instruments. 


\subsubsection{Microinjecting carbon nanotube suspensions into Drosophila embryos}

About 40 min after egg deposition, the CNT suspension was individually injected into the dechorionated embryos glued onto the glass coverslip. A glass needle filled with functionalized CNTs in a watery suspension was inserted into the embryos. Basis for the injection needle was a $1 \mathrm{~mm}$ thick glass capillary (Thin Wall Glass Capillaries TW100F-4; World Precision Instruments Germany GmbH, Friedberg, Germany) which was tapered with a needle puller. Since the tapered needle outlet was closed after pulling the capillary, the tapered end had to be clipped off by applying slight pressure to the cover glass. Maintaining an adequate opening diameter (estimated to tens of micrometers) required a high degree of experience in handling injection needles. To locate the embryos during manual microinjection within the assembly (Fig. 3.5. A-B), we used a stereo light microscope (OLYMPUS IX70; Olympus K. K., Tokyo, Japan) (Fig. 3.4, B). With a microinjector (Transjector 5246 and TransferMan ${ }^{\circledR}$ NK2; Eppendorf AG, Hamburg, Germany), the contents of the glass needle (about $1-5 \%$ of the embryo volume) were released into the embryo by air pressure. Here, it proved to be practical to regulate the used air pressure manually again.
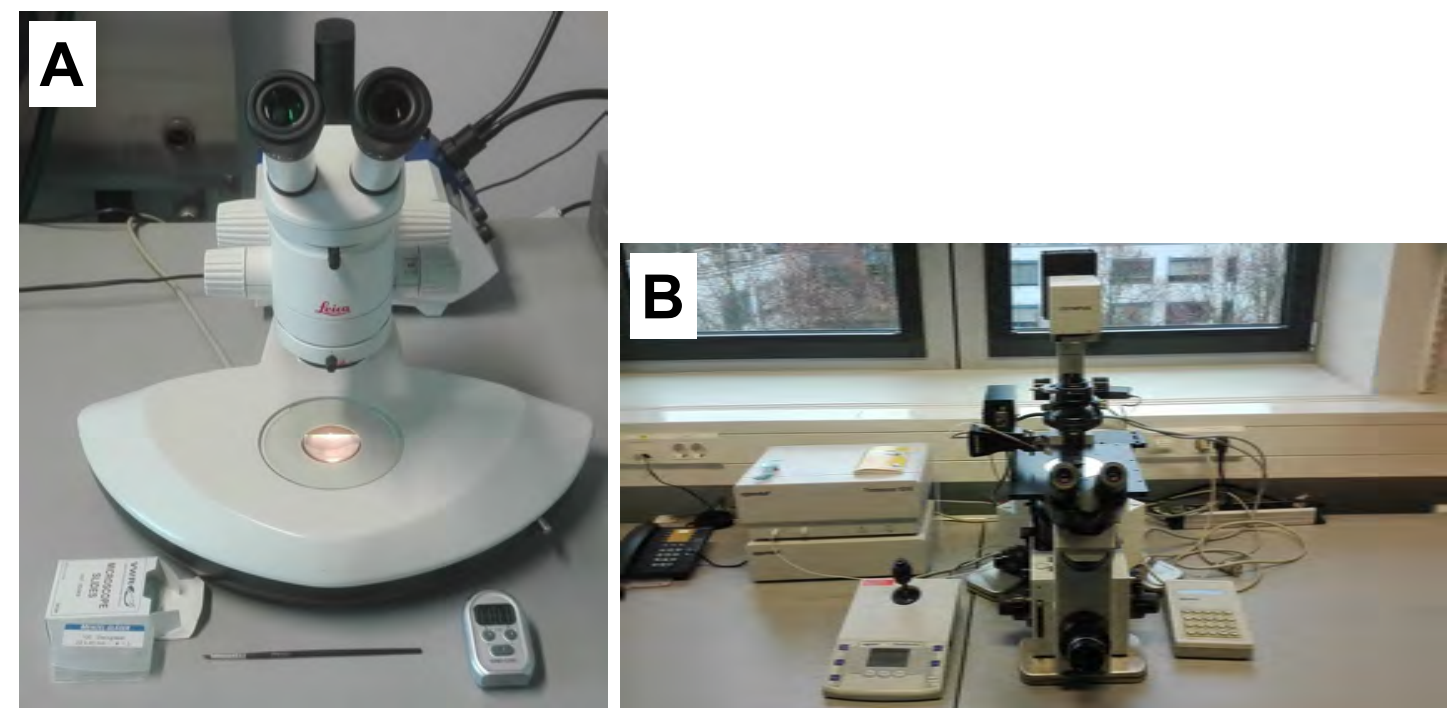

Figure 3.4. Stereo light microscopes used during Drosophila embryo preparation and microinjection. Stereo light microscope (A) was used to harvest the dechoronated embryos from the grid and to place the embryos on the glass coverslips. For the actual microinjection procedure, we used a stereo light microscope which allows for using a micrometer-sized injection needle (B).

When a new CNT sample was used for the first time, an adequate concentration was determined iteratively by alternating injection and imaging, since differences in the spatial distribution of fluorescent CNTs within the embryos were observed during later imaging despite using the same protocol for CNT preparation. In most cases it turned out that an adequate concentration of functionalized CNT suspension can be 
achieved by using a dilution factor of 1:50.

By studying possible influencing parameters on the later distribution of CNTs in imaging, it was found that the distribution of individual fluorescent CNTs within embryos depends on the needle position during microinjection. For that purpose, we have examined and compared two basic approaches to guide the microinjection needle (Thin Wall Glass Capillaries TW100F-4; World Precision Instruments Germany GmbH, Friedberg, Germany) into the Drosophila embryo (Fig. 3.5), and then chose the one that led to a more homogeneous distribution of CNTs in the cortex.

In the first approach, the needle was unloaded along the long axis (anteroposterior axis) while being pulled out (Fig. 3.5, A, C.a, D.a). However, this procedure did not show the desired effect of distributing CNTs more homogeneously over the embryo. The variant in which the needle was introduced along the short axis (transverse axis) turned out to be more promising (Fig. 3.5. B, C.b, D.b). During unloading of the injection needle in this case, a more homogeneous distribution of fluorescent CNTs in the embryo was observed in most cases. Additionally, the interesting field of view (FOV) is filled out more efficiently when injecting along the short transverse axis.

For the injection along the short axis as well as along the long axis, the angle of the needle to the planes of principal axes was chosen as flat and as possible (approximately $20^{\circ}$ ) and as close to the coverslip as possible in order to increase the chances to find most of the CNTs in the cortical region. Regarding the mechanical behavior during injection, no difference was observed between the two embryo groups (His-EGFP and His-EGFP/kin-5-Halo). It could happen that embryonic tissue caused the needle to clog or break and a new needle with CNTs had to be used, making a continuous injection challenging. Furthermore, the microinjection procedure required a high degree of practice in the interaction between the microinjector, the glass needle and the embryos. 
A

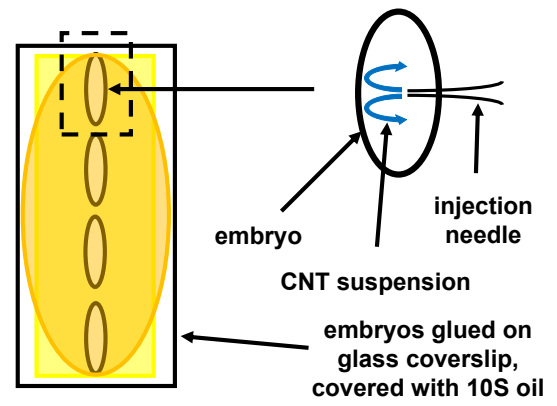

B

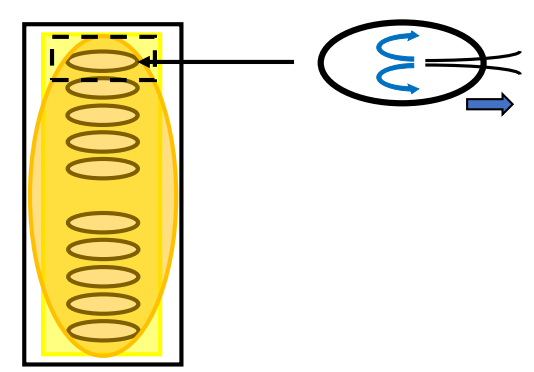

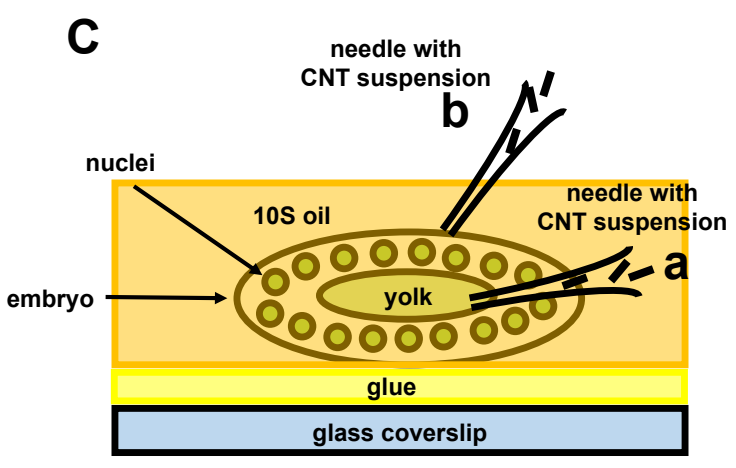

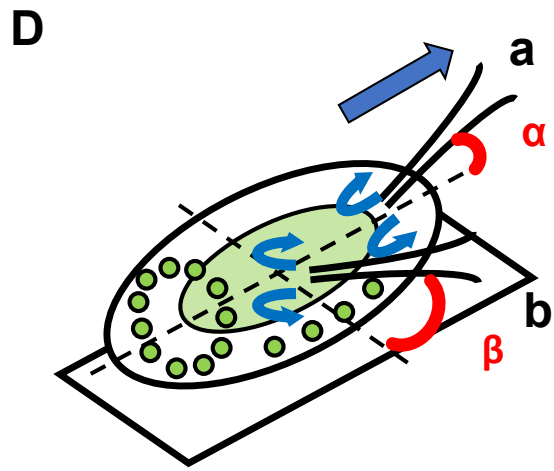

Figure 3.5. Schematic of the microinjection process of CNTs into Drosophila embryos. Panels (A) and (B) show the top views of two different arrangements of Drosophila embryos on a glass coverslip. Top view of arrangement of Drosophila embryos on a glass coverslip for CNT suspension being microinjected into the embryo along short transverse (A) and the long anteroposterior axis (B). (C) Side view with all the layers as they appear during microinjection of CNT suspension and during fluorescence imaging. Nuclei are at cortical periphery and the yolk cell located amidst the Drosophila embryo. D. In both cases of the microinjection needle containing [CNT suspension is inserted at an angle of about $20^{\circ}$ or less (red, $\alpha$ and $\beta)$. Needle positions during microinjection are aligned with long (C.a and D.a) and short (C.b and D.b) embryo axis. 



\section{Multimodal imaging}

\section{Statement}

The following chapter is a quote of the peer-reviewed journal publication (Chap. 8).

Contributions of Constantin Kohl to the above-mentioned journal publication include:

- optimization of the detection path for experiments with CNTs in syncytial Drosophila embryos,

- conceptionalization of the measurement protocols used in the journal publication,

- acquiring the data used in the above-mentioned paper (except figure 5),

- writing the draft of the manuscript.

\subsection{Experimental setup for multimodal imaging}

All fluorescence studies presented in this work are performed with a custom-built inverted fluorescence microscope. The setup can be divided into two main parts: a excitation section and a detection section. 11 In the excitation section, multiple lasers are coupled into a vertical objective to excite fluorescence. We image NIR fluorescence of CNTs using a indium gallium arsenide (InGaAs) complementary metal-oxidesemiconductor (CMOS) camera. For simultaneous VIS imaging we use a low-noise electron multiplying charge-coupled device (EMCCD $)$. With an IR spectrometer, we set the wavelength of the titanium-sapphire (Ti:Sapph) laser for NIR fluorescence excitation of CNTs. A detailed list of the optical components can be found in table 4.1 .

\footnotetext{
${ }^{1}$ The excitation part of the setup was built by Dr. Alok D. Weßel, who also performed pilot studies on which the present work is based [133. The detection part was optimized for imaging in cells and Drosophila by Constantin Kohl.
} 


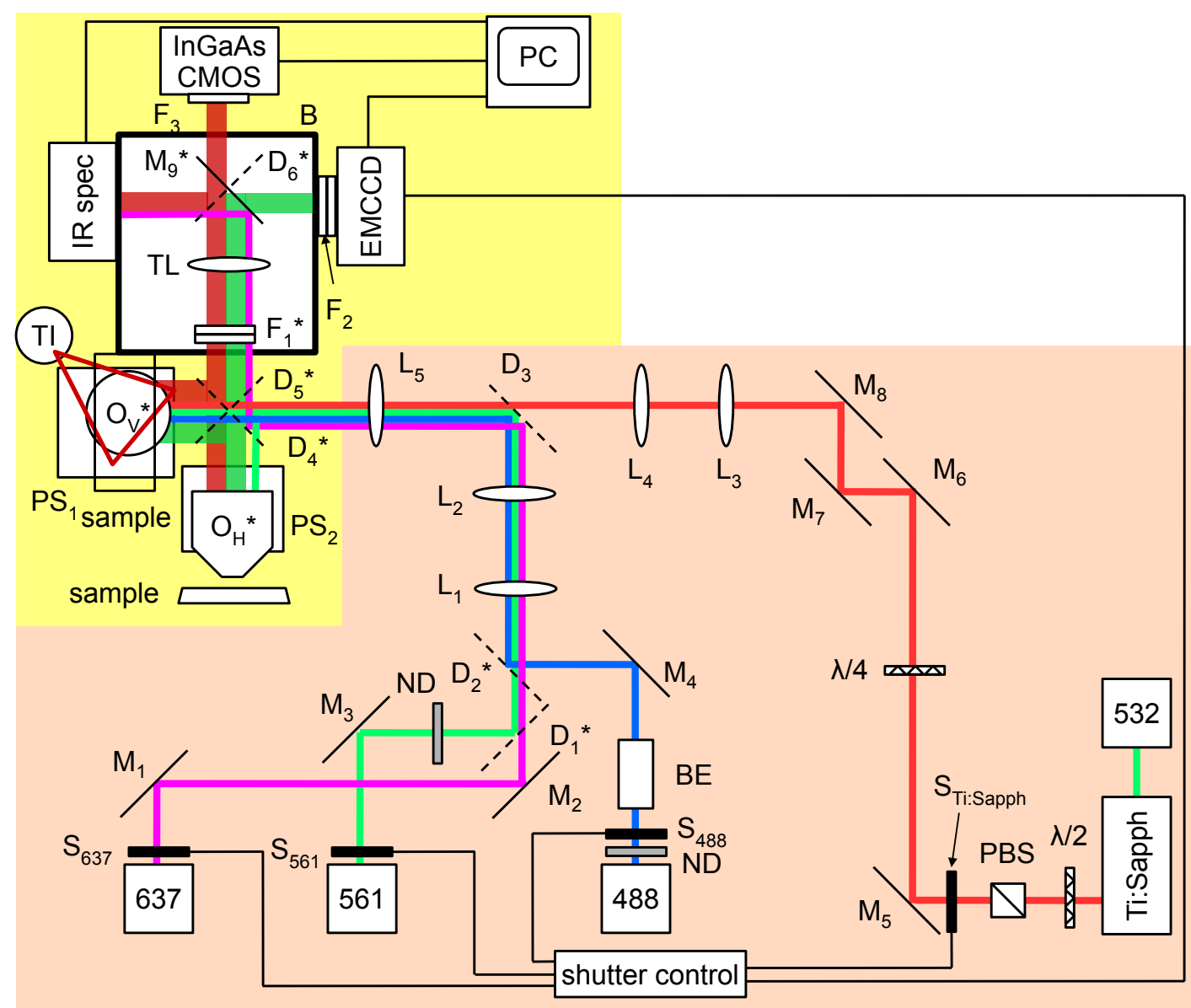

Figure 4.1. Schematic of custom-built setup for multimodal NIR and VIS imaging and NIR spectroscopy. Excitation system (orange shading), detection system (yellow shading). Excitation laser paths are drawn as colored lines. Lasers are shuttered by electromechanical shutters (S). Beams are steered and aligned by broad-band silver mirrors $\left(\mathbf{M}_{1-8}\right)$, and combined by dichroic mirros (D). Beam diameters are expanded by a beam expander $(\mathbf{B E})$ and telescope lens combinations $\left(\mathbf{L}_{1} / \mathbf{L}_{2}, \mathbf{L}_{3} / \mathbf{L}_{4}\right)$. Intensity is regulated by neutral density filters (ND), and a combination of half-wave plate $(\lambda / \mathbf{2})$ and polarizing beam splitter (PBS) for the Ti:Sapph laser. A quarter-wave plate $(\lambda / 4)$ serves to produce circular polarization. Some optical elements (marked with an asterisk) can be brought in or taken out of the beam path. Emission paths are marked by broad green and red lines. A light-tight box (B) encloses the detection optics. Additional filters $\left(\mathbf{F}_{1}^{*}, \mathbf{F}_{2}, \mathbf{F}_{3}\right)$ are used in the emission path. (Tab. 4.1) provides a detailed list of the optical elements. 


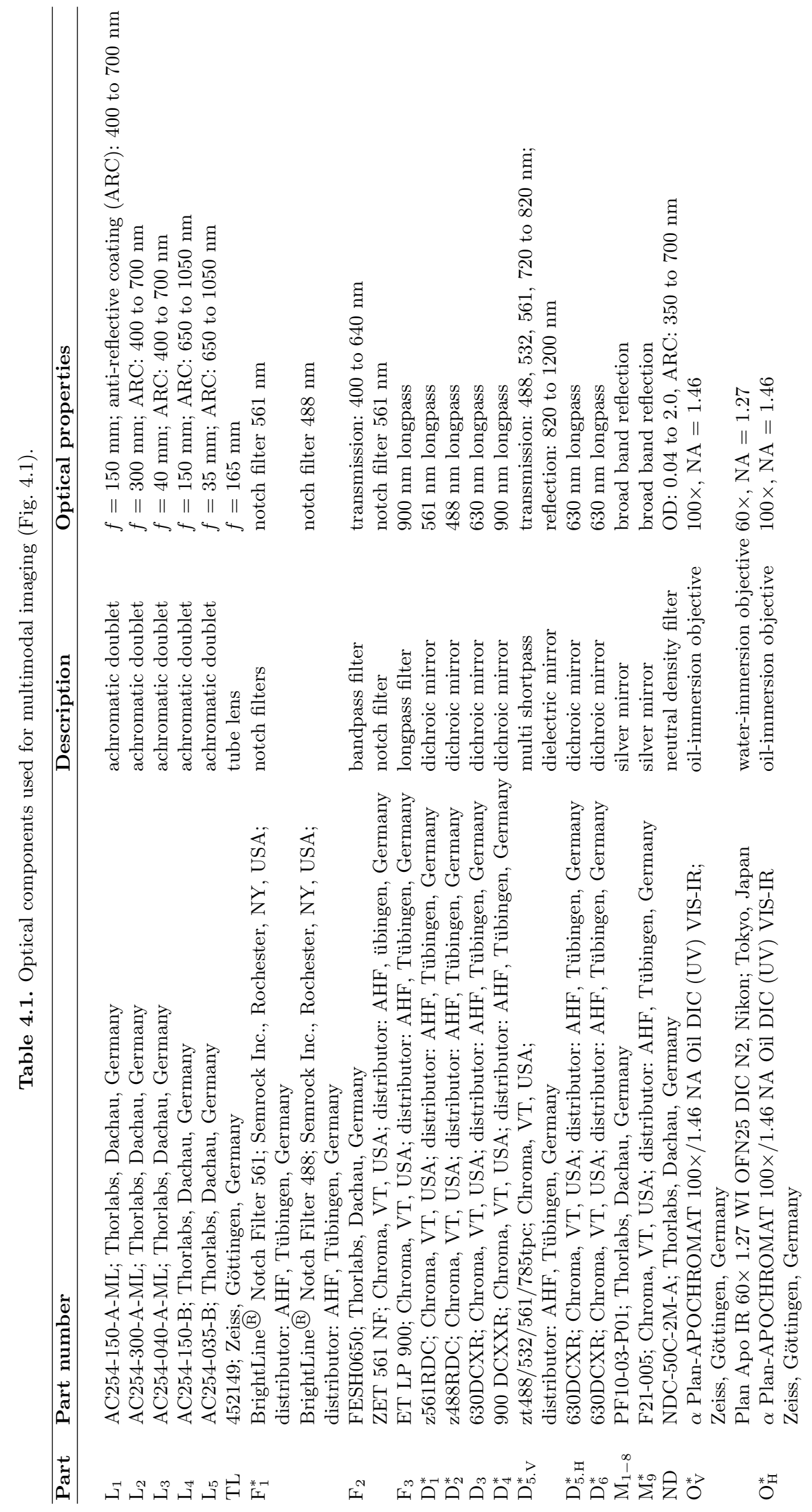




\subsubsection{Excitation system}

\section{Visible fluorescence excitation}

VIS fluorescence (here of His-EGFP) is excited by a $488 \mathrm{~nm}$ laser (Obis $488 \mathrm{~nm}$, cw, $50 \mathrm{~mW}$; Coherent Inc., Irvine, CA, USA). A mechanical shutter directly in front of the laser controls this excitation light. A custom-built control panel (shutter control) controls all electro-mechanical shutters in the setup. The intensity of the laser beam is controlled by a neutral density filter wheel (optical density (OD): 0.04 to 2.0, ARC: 350 to $700 \mathrm{~nm}$, NDC-50C-2M-A; Thorlabs, Dachau, Germany) directly following the shutter. Emission light then passes through a $2-8 \times$ beam expander $(11234858$ Beam-Expander; Rodenstock, Munich, Germany) bringing the beam diameter to about $15 \mathrm{~mm}$. The blue laser is then coupled into a telescope system $\left(\mathrm{L}_{1}, f=150 \mathrm{~mm}\right.$, ARC: 400 to $700 \mathrm{~nm}$ and $\mathrm{L}_{2}, f=300 \mathrm{~mm}$, ARC: 400 to $700 \mathrm{~nm}$ ) via a silver mirror $\mathrm{M}_{4}$ (broad-band reflection) and a dichroic mirror $\mathrm{D}_{2}^{*}$ (488 $\mathrm{nm}$ longpass) to collimate the laser beam after bringing the beam diameter to about $10 \mathrm{~mm}$. Excitation light then passes $\mathrm{D}_{3}$ (630 nm longpass) and is collimated by $\mathrm{L}_{5}(f=35 \mathrm{~mm}$, ARC: 650 to $1050 \mathrm{~nm})$. Using exchangeable mirrors at position $\mathrm{D}_{5}^{*}\left(\mathrm{D}_{5 . \mathrm{H}}^{*}, 630 \mathrm{~nm}\right.$ longpass and $\mathrm{D}_{5 . \mathrm{V}}^{*}$, transmission: 488, 532, 561, 720 to $820 \mathrm{~nm}$, reflection: 820 to $1200 \mathrm{~nm}$ ) makes it possible to switch between a horizontally mounted and a vertically mounted objective. The horizontal objective ( $\alpha$ Plan-APOCHROMAT $100 \times / 1.46$ NA Oil DIC (UV) VIS-IR; Zeiss, Göttingen, Germany) provides a simpler beam path and slightly better light collection and is used for imaging in closed sample chambers, the vertical objective ( $\alpha$ Plan-APOCHROMAT 100×/1.46 NA Oil DIC (UV) VIS-IR; Zeiss, Göttingen, Germany) makes it possible to observe cells or tissues in open containers, such as Petri dishes. The experiments described here used the vertical objective which is mounted on top of a $x-y$-z piezo stage (NanoCube P611.3S, Physik Instrumente $\mathrm{GmbH} \& \mathrm{Co}$. KG; Karlsruhe, Germany). Coarse adjustment of the vertical sample stage uses a mechanical $x-y$ - $z$ stage (M-562-xyz; Newport, Irvine, CA, USA) below the piezo stage.

\section{Carbon nanotube near-infrared fluorescence excitation by green laser}

NIR fluorescence of a particular class of CNTs andVIS red fluorescence of beads used for calibration is excited by a high-power $561 \mathrm{~nm}$ laser (Cobolt Jive 500, $500 \mathrm{~mW}$; Cobolt, Solna, Sweden). The laser is switched on and off with a mechanical shutter. The intensity of the green laser is controlled via a neutral density filter wheel (ND, OD; 0.04 to 2.0, ARC: 350 to $700 \mathrm{~nm}$, NDC-50C-2M-A; Thorlabs, Dachau, Germany). After being reflected by silver mirror $\mathrm{M}_{3}$ and dichroic $\mathrm{D}_{1}^{*}$ (561 $\mathrm{nm}$ longpass), the path of green excitation light $(561 \mathrm{~nm})$ is coupled into the telescope system $\mathrm{L}_{1}, \mathrm{~L}_{2}$ for expansion to about $10 \mathrm{~mm}$. After this, the green laser is aligned collinear with the light path of the blue laser using silver mirrors $\mathrm{M}_{3}$ and $\mathrm{M}_{4}$ as well as the dichroic mirror $\mathrm{D}_{2}^{*}$. 


\section{Carbon nanotube near-infrared fluorescence excitation with near-infrared laser wavelength}

Some classes of CNTs can be excited in the NIR, For that purpose we use a tunable Ti:Sapph laser (770 nm, MIRA 900-F 710-810 nm; Coherent Inc., Santa Clara, CA, USA). The Ti:Sapph laser is pumped by a green laser (Verdi-V12 $532 \mathrm{~nm} 12 \mathrm{~W}$; Coherent Inc., Irvine, CA, USA). After being reflected by a set of silver mirrors $\mathrm{M}_{5} \mathrm{M}_{8}$, the light passes through a telescope system $\left(\mathrm{L}_{3}, f=40 \mathrm{~mm}\right.$, ARC: 400 to $700 \mathrm{~nm}$ and $\mathrm{L}_{4}, f=150 \mathrm{~mm}$, ARC: 650 to $1050 \mathrm{~nm}$ ) for beam collimation.

\section{Setting of the titanium-sapphire laser wavelength}

In order to find the optimal wavelength for excitation of CNT NIR fluorescence, the wavelength of the Ti:Sapph laser is tuned. To set the laser wavelength, we use an IR spectrometer (Acton spectrograph Sp-2150i and InGaAs detector OMA V 512-1.7; Princeton Instruments, Trenton, NJ, USA) and two reference laser wavelengths 561 $\mathrm{nm}$ and $637 \mathrm{~nm}$ (Cube $637 \mathrm{~nm}$, cw, $25 \mathrm{~mW}$; Coherent Inc., Irvine, USA) that provide peaks in a sharply defined emission window. After being reflected by silver mirrors $\mathrm{M}_{1}$ and $\mathrm{M}_{2}$, the red laser $(637 \mathrm{~nm})$ is coupled into the beam path of the green and the blue lasers $\left(561 \mathrm{~nm}\right.$ and $488 \mathrm{~nm}$ ). The dichroic mirror $\mathrm{D}_{4}^{*}$ (900 nm longpass) reflects excitation light from the visible lasers into the horizontal objective $\mathrm{O}_{\mathrm{H}}^{*}$. The defined spectral lines of the two reference lasers are measured by the IR spectrometer and serve as a two-point calibration for tuning the Ti:Sapph laser (Fig. 4.5)).

\subsubsection{Detection system}

The detection side of our setup is depicted in (Fig. 4.2). For experiments using open chambers, such as with cells or Drosophila embryos in Petri dishes, we use the vertically oriented objective, and the sample is placed on a horizontal custom-built sample stage as shown in (Fig. 4.3). Fluorescence emission is detected by two cameras recording NIR fluorescence and VIS fluorescence simultaneously. Multiple imaging channels were, for instance, used to separate the slow nuclear dynamics in Drosophila embryos in the VIS from the fast dynamics of NIR fluorescent CNTs in Drosophila embryos synchronously. NIR fluorescence from CNTs is imaged using an InGaAs CMOS camera (XEVA-FPA-1.7-320; Xenics, Leuven, Belgium) with a chip size of $320 \times 256$ pixels and a pixel size of $30 \mu \mathrm{m} \times 30 \mu \mathrm{m}$. VIS fluorescence is recorded by an EMCCD camera (iXon DU-888E; Andor Technology, Belfast, UK) with a chip size of $1024 \times 1024$ pixels and a pixel size of $13 \mu \mathrm{m} \times 13 \mu \mathrm{m}$. In this configuration, we image an area of $76.8 \times 96 \mu \mathrm{m}^{2}$ in the NIR channel and $133.12 \times 133.12 \mu \mathrm{m}^{2}$ in the VIS channel. Depending on the organism of interest, we can use two different sample stages during the imaging: one for imaging cells and one for imaging Drosophila embryos (see below). The light collected through the objective is passed through a set of notch filters $\left(\mathrm{F}_{1}^{*}\right)$ to remove remaining excitation light. A tube lens focuses the fluorescence light onto the cameras or onto the spectrometer. Before the emission light reaches the detectors, it arrives at a switch point $\left(\mathrm{M}_{9}^{*}, \mathrm{D}_{6}^{*}\right)$ where we can choose among three options for light collection: Emission light can either directly pass to the InGaAs CMOS camera for NIR detection, or it can be fully reflected into the IR 
spectrometer (Acton spectrograph Sp-2150i and InGaAs detector OMA V 512-1.7; Princeton Instruments, Trenton, NJ, USA), or the emission light can be split by a dichroic mirror $\left(\mathrm{D}_{6}^{*}\right)$ to obtain VIS images and NIR images simultaneously.

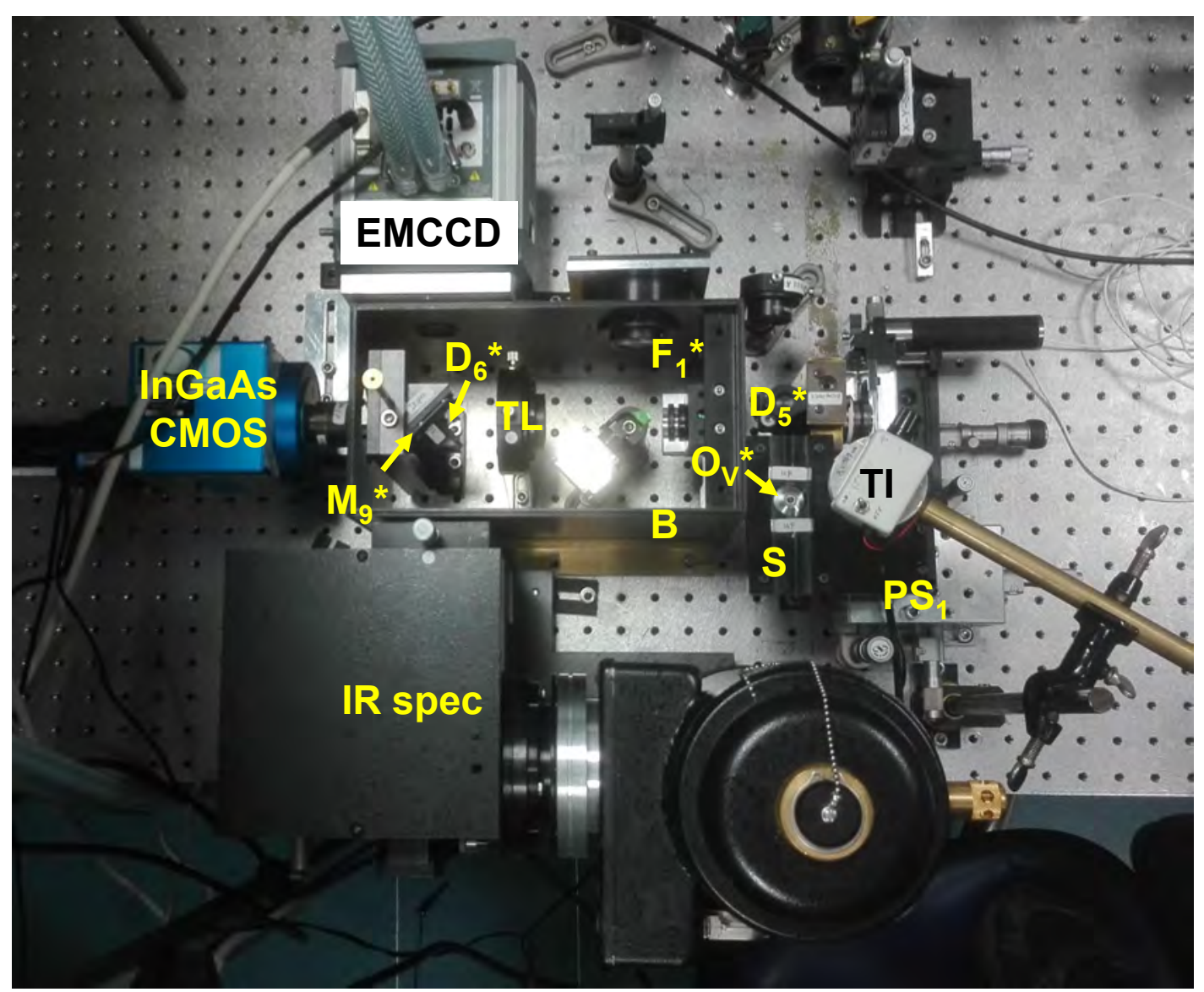

Figure 4.2. Detection section for simultaneous $\mathrm{VIS}$ and $\mathrm{NIR}$ imaging: The fluorescence light from the sample on the horizontal stage $(\mathbf{S})$ is collected by the vertical objective $\left(\mathbf{O}_{\mathbf{V}}^{*}\right)$, guided by a silver mirror below the objective $\left(\mathbf{O}_{\mathbf{V}}^{*}\right)$ onto a dichroic mirror $\left(\mathbf{D}_{5}^{*}\right)$ which transmits excitation light and reflects VIS and NIR emission light into the detector box (B). A set of notch filters $\left(\mathbf{F}_{1}^{*}\right)$ suppresses remaining excitation light. The light then passes the tube lens (TL) before it arrives at a switch position $\left(\mathbf{M}_{9}^{*}, \mathbf{D}_{6}^{*}\right)$, that either guides the emitted light fully into the IR spectrometer (IR spec) or fully into the InGaAs CMOS camera for NIR fluorescence detection (InGaAs CMOS), or splits VIS from NIR light by a 630-nm longpass filter into the InGaAs camera (InGaAs CMOS) for NIR fluorescence detection and the EMCCD camera (EMCCD) for VIS fluorescence detection. For simple transillumination the sample is illuminated by a NIR diode array lamp (TI) with adjustable intensity. The sample stage is mounted on a 3-axis piezo stage $\left(\mathbf{P S}_{1}\right)$ for fine positioning, which, in turn, is mounted on a 3-axis micrometer stage for coarse positioning $\left(\mathbf{P S}_{1}\right)$. Optical elements are listed in (Tab. 4.1).

To measure the dynamics of CNTs in Drosophila embryos, we recorded NIR fluorescence of CNTs simultaneously with EGFP fluorescence of histones. After localizing the sample and identifying an area of interest in the NIR channel with a NIR trans-illumination lamp (Stanley 111R IR-Diode, AN1111R; Stanley Electric Co., Ltd, Tokyo, Japan), we simultaneously excited EGFP fluorescence with the $488 \mathrm{~nm}$ laser and CNT NIR fluorescence with the NIR laser light set to $770 \mathrm{~nm}$. We used the fluorescence of His-EGFP to identify a Drosophila embryo at the right stage of development and select the focal plane that contains the nuclei near the embryo 
cortex. We sampled fluorescence at different rates for VIS and NIR. Since CNTs do not photobleach, we used a sample rate of $f=10 \mathrm{~Hz}$ for the NIR imaging. Since EGFP does photobleach, we applied intermittent illumination using the shutter in front of the blue laser. To synchronize shutter and camera, we controlled the shutter from the EMCCD camera's "fire" output which, via a shutter driver, opened the shutter for $t=1 \mathrm{~s}$ after each $T=20 \mathrm{~s}$. EMCCD camera and shutter are controlled by Micro-Manager software [144].

\section{Sample stages}

Our setup allows us to mount two different sample stages adapted to the specific requirements of commonly used biological systems such as cells or Drosophila embryos. Since many cell lines during in vivo experiments demand exact temperature control, the sample stage used for experiments with cells can be thermalized by a Peltier element below the sample holder (Fig. $4.3 \mathrm{~A}, \mathrm{~B}$ ). The sample is in contact with an aluminum plate which, in turn, guides the heat from the Peltier element to the Petri dish, keeping the cells at $\approx 37^{\circ} \mathrm{C}$. Drosophila embryos can be imaged at room temperature and require a simpler sample holder (Fig. 4.3 C, D). For imaging, embryos were lined up on a glass coverslip $\left(22 \times 40 \mathrm{~mm}^{2}\right)$ and mounted onto the sample stage fitting into a groove of $22 \mathrm{~mm}$ in width. The cover slip is fixed on the stage by two pairs of rare-earth magnets below the sample stage and two rare-earth magnetic bars on top of the cover slip.
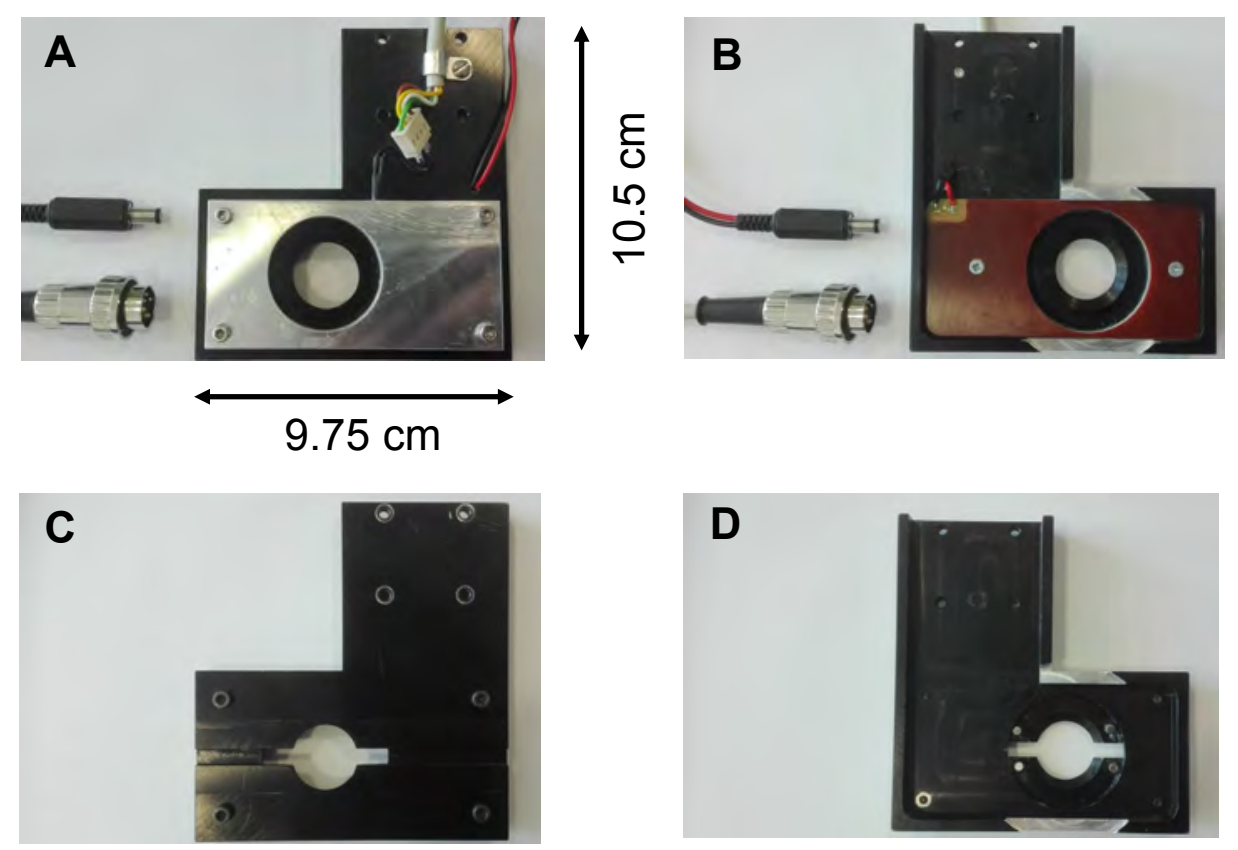

Figure 4.3. Sample stages for experiments with cells (A, B) and Drosophila embryos (C, D). A. Top view of the sample stage for experiments with cells. To hold the Petri dishes, an aluminum plate for temperature control of the Petri dish is screwed to the main corpus of the stage and heated with a Peltier element. B. Bottom view of the sample stage for experiments with cells with the circuit board for the Peltier element. C. Top view of the sample stage for experiments with Drosophila embryos. D. Bottom view of the sample stage for experiments with Drosophila embryos with two pairs of rare-earth magnets glued to the stage for fixing the sample. 


\section{Electron multiplying charge-coupled device camera for detecting the visible channel}

In this work we image the dynamical morphological changes of nuclei of Drosophila embryos during successive developmental stages in the VIS spectrum. For imaging the nuclear phases of Drosophila we use a low-noise EMCCD camera. Here we want to give a short outline to reproduce the data acquired within this work. Initially charge-coupled device (CCD) sensors have been invented as a data storage device, but soon their high sensitivity towards photons was recognized as high potential for imaging studies [145, 146]. Their wide sensitivity range in the VIS] range [147] makes them optimal dectors for VIS fluorescence of EGFP.

On the surface of a semiconducting material potential wells containing charges are moved along the surface of the semiconductor transporting these stored charges [145. The principle of image acquisition with an EMCCD is based on averlanching electronic charge in a CCD [148]. An EMCCD, as used in our work, comprises of five sections: a light-absorbing image array being about the same size as a subsequent light-reflecting storage array, a shift register, a multiplication register and on-chip charge to voltage converting output (Fig. 4.4] [148].

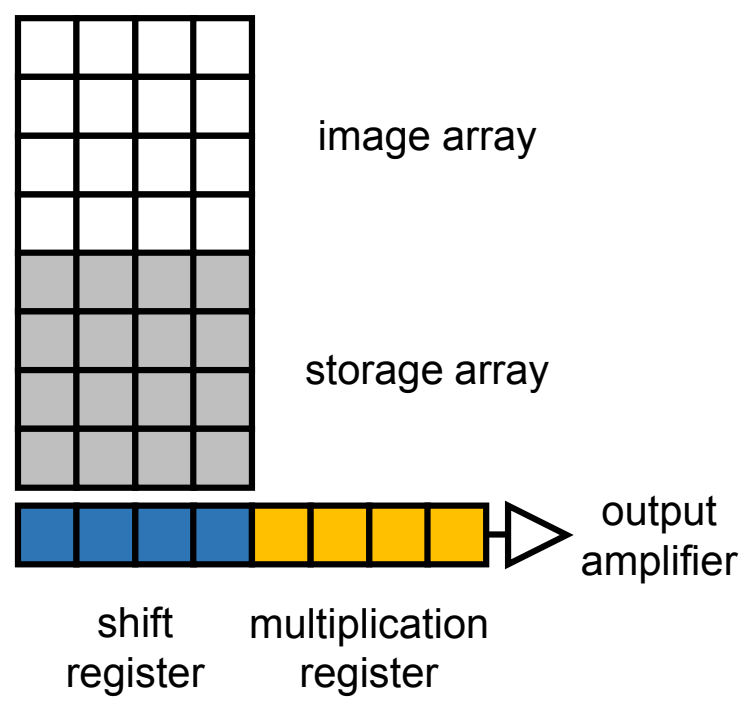

Figure 4.4. Schematic of the basic EMCCD chip design. During the readout procedure, the depicted areals of an EMCCD are image section (white), storage section (gray), shift register (blue), multiplication register (yellow) and output amplifier (triangle). Figure altered after [148].

In an EMCCD image acquisition and image readout are two sequential processes. When light hits the image array during image acquisition photons create an electronic image. Readout of this charge pattern occurs by vertical shift into the readout register. There the lowermost pixel row is then shifted horizontally towards the output amplifier. Before the first pixel finally reaches the output, impact ionization in the multiplication register causes enhancement of the electronic charge before passing the output amplifier circuit. The above-mentioned vertical shift procedure repeats until each pixel in the whole image array is read out as outlined above. During the imaging experiments performed in this work, we make use of the so 
called frame transfer acquisition mode. Activating this mode, reduces the odds of obtaining vertical smearing due to erroneously overwritten pixels. The storage area enables a fast charge transfer without having an increased noise level. A schematic example of an EMCCD capable of being operated in frame transfer mode can be seen in figure 4.4 .

\section{Indium gallium arsenide complementary metal-oxide-semiconductor camera for detecting the near-infrared channel}

For the imaging of optical signals in the NIR, CMOS sensors from InGaAs are often used [149, 150, since these have sensitivity peaks in the range of about $10^{3} \mu \mathrm{m}$ [150]. For imaging NIR fluorescent CNTs we use a camera with such an InGaAs CMOS chip. Unlike charge-coupled devices (CCDs), most functions of CMOS sensors, such as photon-to-electron and electron-to-voltage conversion, are implemented directly on-chip making them more robust than CCDs [151]. Before the InGaAs CMOS camera is used for imaging NIR CNTs, the sensor must be calibrated in a two point calibration. For that, we darken the camera aperture and record a so-called dark image as a reference image. Then, we open the camera aperture and record a light image. For this, we use a custom-built NIR diode lamp (Stanley 111R IR-Diode, AN1111R; Stanley Electric Co., Ltd., Tokyo, Japan) emitting at $950 \mathrm{~nm}$ to carefully and homogeneously illuminate the whole FOV. It is important not to cut the upper limit of the dynamic range by fully saturating the pixels and thereby shorten the dynamic range. After calibration, we use the InGaAsICMOS camera for acquisition of NIRICNT fluorescence at a frequency of $10 \mathrm{~Hz}$. Here, we use the high-gain acquisition mode for imaging in order to collect as much light as possible from the CNTs [152.

\section{Infrared spectrometer}

In this work, we use the IR spectrometer (10) for setting the Ti:Sapph laser wavelength. For setting the Ti:Sapph laser wavelength, we use two wavelength reference points (561 nm and $637 \mathrm{~nm}$ ) defined by incident laser peaks (Fig. 4.5). With these laser wavelengths we obtain two reference points which can be used to read a third peak in the spectrum, gained by the IR spectrometer (see figure Fig. 4.5). 


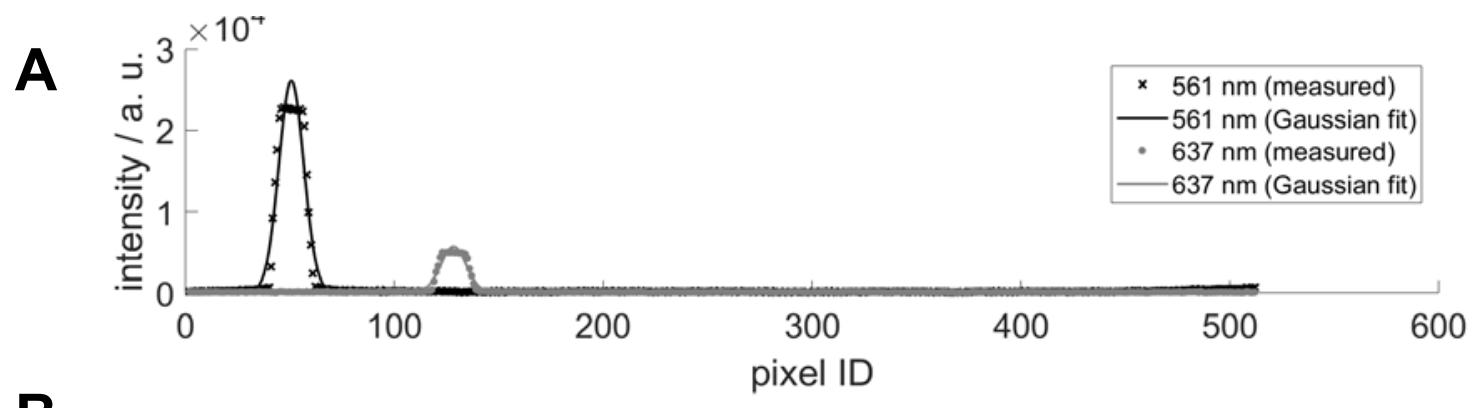

B
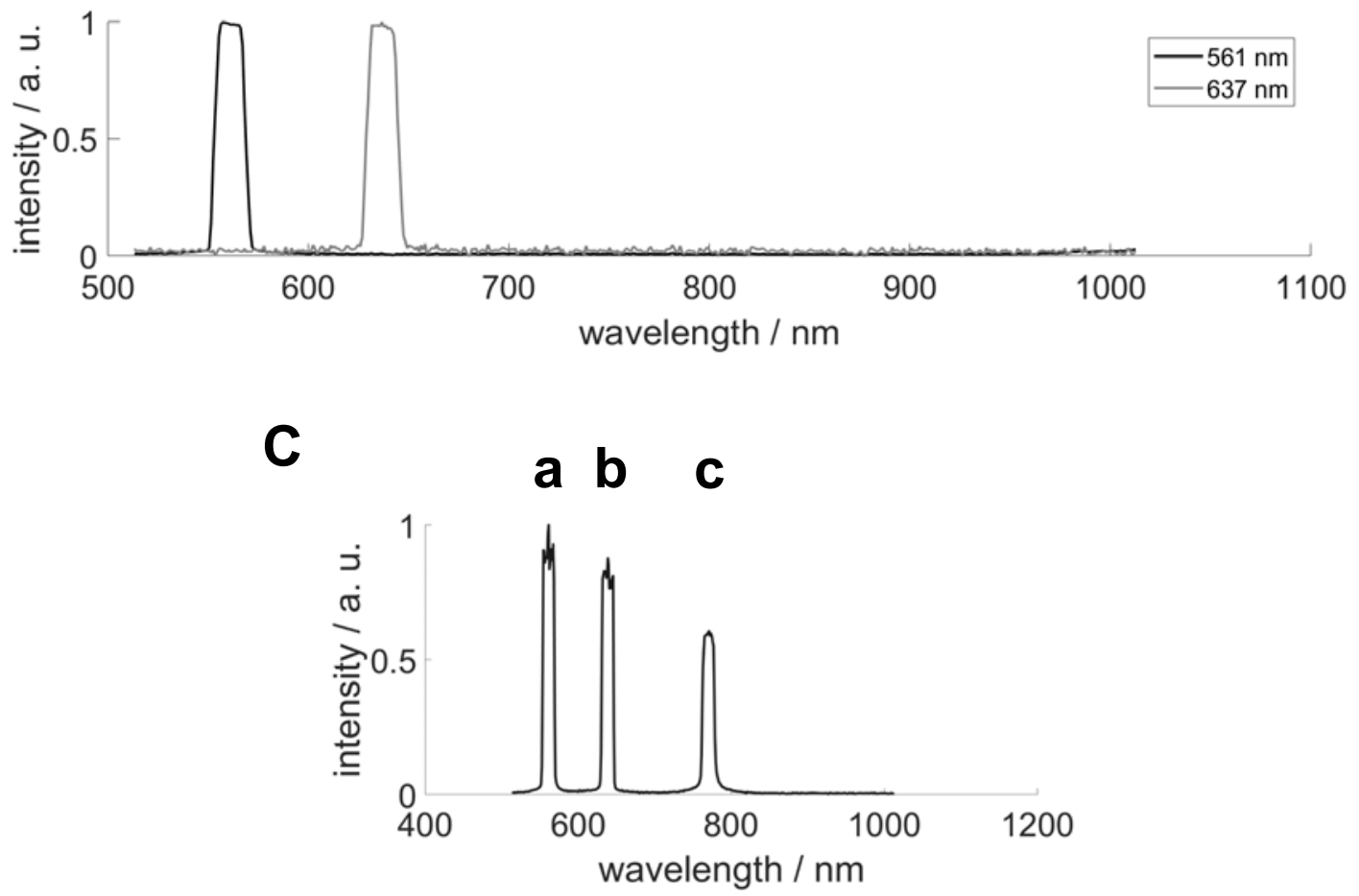

Figure 4.5. Resulting spectral peaks, measured by the IR spectrometer for setting the wavelength of the tunable Ti:Sapph laser. The wavelength of the Ti:Sapph laser is aligned by a two-point method. We use two distinct lasers to obtain peaks at defined wavelengths: $561 \mathrm{~nm}$ (a) and $637 \mathrm{~nm}$ (b). By this, we find the fitting NIR excitation wavelength for CNT excitation with the Ti:Sapph laser at $770 \mathrm{~nm}$ (c). 


\section{5}

\section{Characterizing optical properties of carbon nanotubes in living Drosophila embryos}

\subsection{Excitation of carbon nanotube fluorescence in living Drosophila embryos}

In the course of our studies it turned out that our batch contains two populations of CNTs. We found out that by using a green laser $(561 \mathrm{~nm}$, Cobolt Jive 500, $500 \mathrm{~mW}$; Cobolt, Solna, Sweden) or a Ti:Sapph laser (MIRA 900-F 710-810 nm; Coherent Inc., Santa Clara, CA, USA) with a wavelength range tunable between 710 and 810 $\mathrm{nm}$, we can excite CNT fluorescence. In order to yield optimal CNT fluorescence, we excite CNTs in living Drosophila embryos sequentially with both lasers.

We used two lasers (561 nm and $770 \mathrm{~nm}$ ) to excite as much CNTs as possible during imaging in living Drosophila embryos.

The signal-to-noise ratio of NIR signals of CNTs excited at $770 \mathrm{~nm}$ was $31 \mathrm{~dB}$, in the case of $561 \mathrm{~nm}$ excitation wavelenth signal-to-noise ratio amounted to $36 \mathrm{~dB}$. Interestingly, figure 5.1 shows a higher yield of excited CNTs exciting the sample with NIR laser light (770 nm, Ti:Sapph) than when exciting the sample with 561 $\mathrm{nm}$. To obtain larger sample sets, we decided to use $770 \mathrm{~nm}$ excitation wavelength because of the higher count of detectable CNTs of this class. 
A

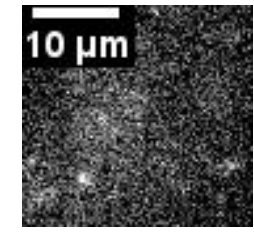

D

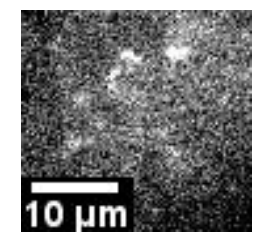

B

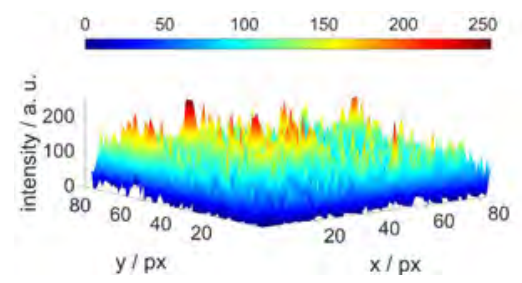

E

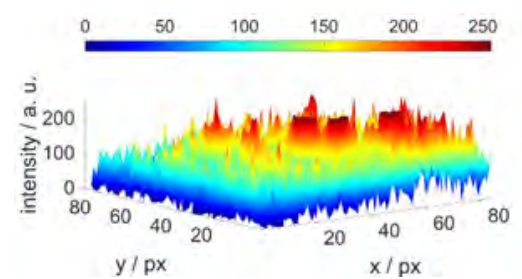

C

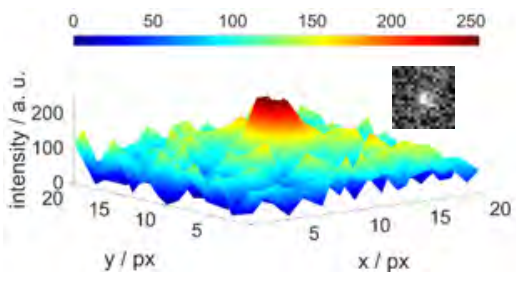

$\mathbf{F}$

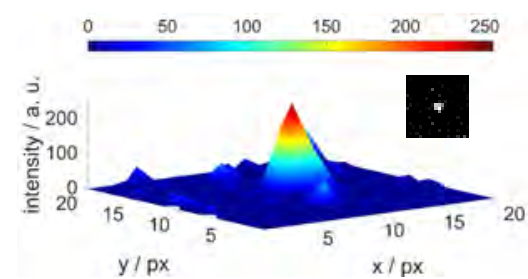

Figure 5.1. Comparison of two laser wavelengths to excite CNTINIR fluorescence in the same sample. A. CNT fluorescence signals under $561 \mathrm{~nm}$ illumination. B. 2D intensity heat plot of CNT [NIR fluorescence (A). C. Zoom of an individual CNT excited by $561 \mathrm{~nm}$. D. CNT fluorescence signals under $770 \mathrm{~nm}$ illumination. E. 2D intensity heat plot of CNT NIR fluorescence signal (D). From the heatplots in (B) and (E), it is evident that fluorescence light was more intense, when CNTs were excited with the NIR laser. F. Zoom of an individual CNT excited by $770 \mathrm{~nm}$. Reproduced from "Near-infrared fluorescence imaging of single-walled carbon nanotubes in living organisms", which has been submitted to Review of Scientific Instruments.

We benefit from the tunable wavelength range of Ti:Sapph laser to set the optimal excitation wavelength of our CNT sample. We find a peak in CNT fluorescence emission at $770 \mathrm{~nm}$ by systematically setting the Ti:Sapph laser wavelength to $770 \mathrm{~nm}$ according to (Chap. 4.1.1) using [CNT spectral information from literature [121 132.

Just as important as the knowledge about optimal wavenglength for CNT fluorescence excitation is knowledge of information on the laser power. The laser power has to be set high enough to obtain as much fluorescence as possible but it also must not be too high to not harm embryos during imaging. Hence, we have measured powers of the lasers which are used to excite CNT fluorescence in embryos at distinct points in the excitation part of the setup (Fig. 5.2 and Fig. 4.1, orange shading). 


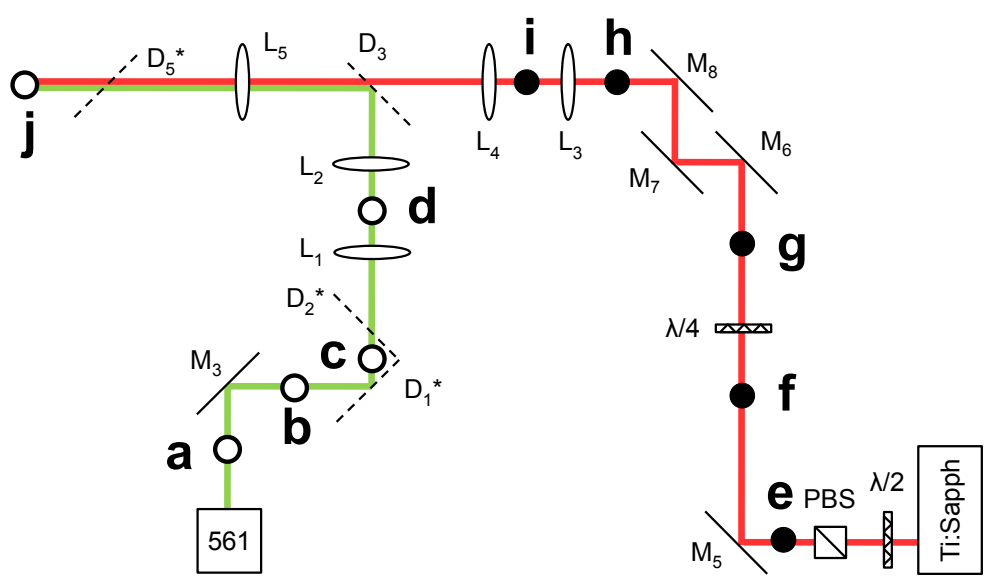

Figure 5.2. Schematic of the measuring points of the powers of the lasers for CNT fluorescence excitation in the imaging setup. Letters label sites of the laser power measurements. Measuring points of the power of the green laser $(561 \mathrm{~nm})$ are marked by empty circles (a)-(d), (j). Points, where the power of the Ti:Sapph laser $(700 \mathrm{~nm})$ was measured are depicted as black circles $(\mathbf{e})-(\mathbf{j})$.

By these measurements we optimized the laser wavelength for illuminating our particular batch of fluorescent CNTs in Drosophila embryos. During the measurement of the laser powers, we coupled all mirrors into the beam path of the $561 \mathrm{~nm}$ laser as they are used for simultaneously illuminating His-EGFP with the blue laser (488 $\mathrm{nm}$ ) during VISt,NIR imaging (Fig. 5.2). In order to measure the maximal power of the green laser $(561 \mathrm{~nm})$, we have measured the laser power in the excitation section without any neutral density filter placed in the beam path.

Table 5.1 lists the power values of the lasers used for CNT excitation.

Table 5.1. Excitation laser powers of the green laser $(561 \mathrm{~nm})$ and the Ti:Sapph laser $(770 \mathrm{~nm})$ measured at particular points in the imaging setup.

\begin{tabular}{lll}
\hline $\boldsymbol{\lambda} / \mathbf{n m}$ & Location & $\boldsymbol{P}_{\boldsymbol{\lambda}} / \mathbf{m}$ \\
\hline \multirow{3}{*}{561} & $\mathbf{a}$ & 405 \\
& $\mathbf{b}$ & 386 \\
& $\mathbf{c}$ & 366 \\
770 & $\mathbf{d}$ & 347 \\
& $\mathbf{e}$ & 1120 \\
& $\mathbf{f}$ & 1000 \\
& $\mathbf{g}$ & 1050 \\
& $\mathbf{h}$ & 640 \\
561 & $\mathbf{i}$ & 570 \\
770 & $\mathbf{j}$ & 287 \\
& & 440
\end{tabular}

It shows, that $71 \%$ of the power of the green laser $(561 \mathrm{~nm})$, measured at (Fig. 5.2 , a), arrives at the vertical objective. In case of the Ti:Sapph laser $(770 \mathrm{~nm})$ only $39 \%$ of the intial laser power, measured at (Fig. 5.2, e), arrives at the vertical objective. 


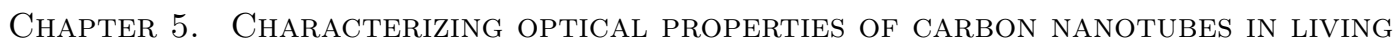
48

Drosophila EMBRYOS

\subsection{Point spread functions of carbon nanotubes in living Drosophila embryos}

A profound inquiry of the optic properties of CNTs is crucial to benefit optimally from the NIR fluorescence of CNTs in Drosophila embryos. Here, we make use of the PSF as a powerful tool to characterize the imaging limitations of a system with regards to resolution of point-like signals like CNTs with lengths of few hundred nanometers. By this we obtain information about the limitations of the NIR fluorescence of CNTs in tissue of Drosophila embryos. Measurement of the PSF gives insight into the sensitivity of CNT fluorescence in distinct layers inside the tissue of Drosophila embryos.

The PSF is a measure for how the optics makes the resulting image of an ideal point source differ from an ideal point source. We define the PSF as a convolution kernel by the convolution [153, 154]:

$$
O=\operatorname{PSF} * I,
$$

where $O$ defines the object matrix and $I$ describes the image matrix.

For the measurement of the PSFs of individual CNTs we used a piezo stage (NanoCube P611.3S; Physik Instrumente GmbH \& Co. KG, Karlsruhe, Germany controlled via E-563 I3N; Physik Instrumente GmbH \& Co. KG, Karlsruhe, Germany) in closed feedback loop to control the scanning of CNTs in multiple depths $d_{i}$ of embryonal tissue (Fig. 5.3).

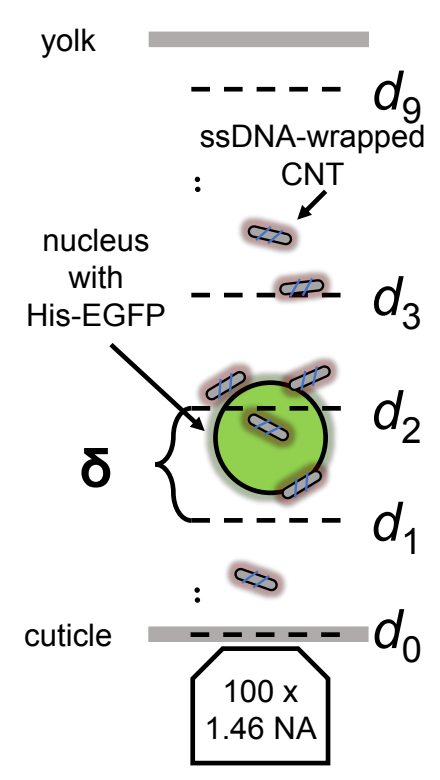

Figure 5.3. Schematic of scanning CNTs in Drosophila embryonic tissue. The sample is scanned in different equidistant layers of embryonic tissue beginning from the actin cortex directed towards the yolk (respective straight lines). Positions $d_{1}, d_{2}, d_{3}, . ., d_{10}$ of the focal plane are depicted as dashed lines. Distance between two neighboring layers is $\delta=5 \mu \mathrm{m}$.

By closing the feedback loop, voltage is the adapting parameter, assuring that the position remains fixed. Measurement of the PSFs in various tissue depths requires previous calibration of the zero plane. First, the $z$ potentiometer of the piezo actor 
is set to its maximum in order to preserve the full scanning range. For practical reasons, we start $z$-sectioning beginning with high values of the $z$ potentiometer of $s_{\max }=100 \mu \mathrm{m}$ scanning in $5 \mu \mathrm{m}$ steps down to $s_{\min }=0 \mu \mathrm{m}$. We define the $z=0$ plane for our measurements by the interface of the coverslip and glue. To find this plane, we use a calibration slide for which we focus the scale. Then, we use the $z$ micrometer screw to correct the piezo stage position for the coverslip thickness $(t=0.17 \mathrm{~mm})$. By this correction, we ensure that the zero plane is identical with the coverslip-glue interface. For calibrating the zero plane for the PSF measurement, we use 1:50 diluted dried-out red fluorescent beads (FluoroMax ${ }^{\mathrm{TM}}, \mathrm{R}$ 600, $0.6 \mu \mathrm{m}$; Thermo Fisher Scientific Life Technologies GmbH, Darmstadt, Germany) which we put on top of the glue. We then focus the piezo stage with the micrometer screw at the mechanical $x-y-z$ stage while keeping the piezo stage for fine tuning at its upmost setting in closed loop mode. In a first calibration approach we tried yellow fluorescent beads which were dried out and then coated with glue. However, the glue did not stick on the surface and, thus, we could not use yellow fluorescent beads during simultaneous recording of CNT's NIR fluorescence and EGFP's VIS fluorescence. Decreasing the concentration of beads was no option, since then the density of beads would have been too low to have enough beads in the FOV. We solved this problem by using beads dried on a glass coverslip and noting the initial position of the piezo stage.

To analyze the individual CNT signals we select rectangular regions of interest with the open-source software Fiji [155] from which we carefully select potential candidates of our CNT signals. With a self-written MATLAB routine we get the maximum intensity of the CNT in the chosen FOV.

Here, we assume an individual CNT as a point-like object. It is convenient to approximate the $\overline{\mathrm{PSF}}$ of a punctate light source by a gaussian kernel [156 158]. Here, we use a 2D Gaussian kernel [159]:

$$
\mathrm{PSF}_{2 \mathrm{D}} \simeq \frac{1}{\sqrt{(2 \pi)^{2} \operatorname{det}(\Sigma)}} \exp \left[-\frac{1}{2}(\mathbf{x}-\boldsymbol{\mu})^{\top} \Sigma^{-1}(\mathbf{x}-\boldsymbol{\mu}),\right]
$$

with the covariance matrix [159]

$$
\Sigma:=\left[\begin{array}{cc}
\sigma_{x}^{2} & \rho \sigma_{x} \sigma_{y} \\
\rho \sigma_{x} \sigma_{y} & \sigma_{y}^{2}
\end{array}\right]
$$

which becomes diagonal in case of uncorrelated $\sigma_{j}$. By plugging definition (Eq. 5.3) into (Eq. 5.2 and by using the position vector $\mathbf{r}:=\left[\begin{array}{ll}x & y\end{array}\right]^{\top}$ of the pixel and the center of the gaussian $\boldsymbol{\mu}:=\left[\begin{array}{ll}\mu_{x} & \mu_{y}\end{array}\right]^{\top}$, the decoupled PSFs of a $2 \mathrm{D}$ system reduces to

$$
\begin{aligned}
& \mathrm{PSF}_{x} \sim \frac{1}{\sigma_{x}} \exp \left[-\left(\frac{x-\mu_{x}}{\sigma_{x}}\right)^{2}\right] \\
& \mathrm{PSF}_{y} \sim \frac{1}{\sigma_{y}} \exp \left[-\left(\frac{y-\mu_{y}}{\sigma_{y}}\right)^{2}\right] .
\end{aligned}
$$


Chapter 5. CharaCterizing optical Properties of CARBOn NANOtUbes IN Living 50

Drosophila EMBRYOS

With OriginPro we fit the PSFs by gaussians in $x$ - and $y$-directions which in turn give us $\sigma$ as a measure for the width of the PSFs of CNTs in embryonal tissue. We average the widths $\sigma_{d_{j}}$ of the gaussian-shaped PSFs measured in tissue depths $d_{j}$ using

$$
\langle\sigma\rangle_{d_{j}}:=\frac{\sigma_{x, d_{j}}+\sigma_{y, d_{j}}}{2}
$$

to obtain a measure for the width of $2 \mathrm{D}$ PSFs of individual CNTs in living Drosophila embryos.

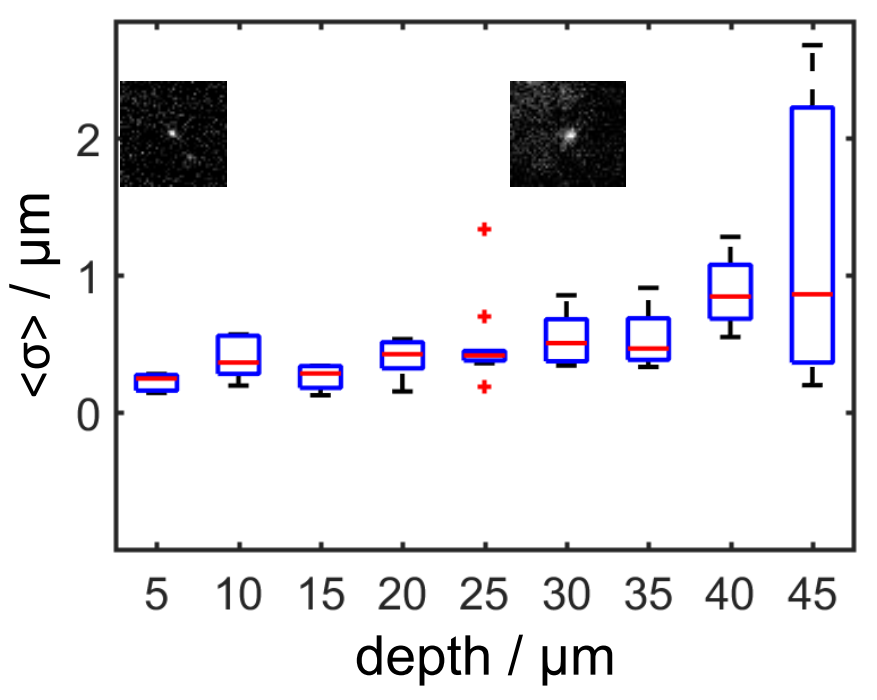

Figure 5.4. Experimental data showing the width distribution of PSFs of individual CNTs in Drosophila embryos. Insets show CNTs 5 and $30 \mu \mathrm{m}$ deep in embryonic tissue. Note the increase in PSF width with increasing tissue depth.

Using (Eq. 5.6) we find values for $\langle\sigma\rangle$ up to a tissue depth of $45 \mu \mathrm{m}$ (Fig. 5.4). Interestingly, we find an increase in the width of the gaussians while going deeper into the tissue. To quantify this increase in $\langle\sigma\rangle_{d_{j}}$ (Eq. 5.6) we look at the medians of for $\langle\sigma\rangle_{d_{j}}$ :

$$
\begin{aligned}
\Delta\langle\sigma\rangle & =\langle\sigma\rangle_{45 \mu \mathrm{m}, \text { median }}-\langle\sigma\rangle_{5 \mu \mathrm{m}, \text { median }} \\
& =0.86-0.25 \mu \mathrm{m} \\
& =0.61 \mu \mathrm{m} .
\end{aligned}
$$

We thus find a discrepancy in the width of the $\mathrm{PSF}$ amounting to $0.61 \mu \mathrm{m}$ in a range of $40 \mu \mathrm{m}$ in the tissue. 


\section{Dynamics of carbon nanotubes in Drosophila embryos}

\subsection{Imaging}

\subsubsection{Imaging protocols}

In this chapter we outline the experimental procedures which led to the results described in chapter (Chap. 6.3). The following protocol is generic, i. e. the underlying procedure is the same for all experiments performed in these chapters.

We found a simple way for conducting both single-nanoparticle experiments and studies of mesoscopic bulk dynamics in living Drosophila embryos.

Changes in paramteres are explicitely mentioned in the following text. Before starting experiments we fix the sample on the vertical sample stage by using two magnetic bars. Each magnetic bar pushes the coverslip with the glued embryos covered in $10 \mathrm{~S}$ oil on top (3.5. A-C) towards the sample stage which has two pairs of rare-earth magnets at the bottom (Fig. 4.3). The next step in each imaging experiment including Drosophila embryos is to locate the embryos on the coverslip.

With a custom-built NIR diode lamp with tunable intensity for transillumination (720 nm, Stanley 111R IR-Diode, AN1111R; Stanley Electric Co., Ltd, Tokyo, Japan) we locate embryo positions in the InGaAs CMOS camera for NIR detection (100 $\mathrm{ms}$ integration time, $320 \times 256$ pixels, $30 \mu \mathrm{m}$ pixel resolution, XEVA-FPA-1.7-320; Xenics, Leuven, Belgium). Sole purpose of the NIR diode lamp is to locate the embryos before the actual simultaneous multi-channel image acquisition. In order to not interfer with the NIR signals from CNTs, we turn off the NIR lamp before starting actual multi-channel image acquisition.

After having located the embryos we use the NIR Ti:Sapph laser to check the CNT yield inside the embryo. This step is crucial, since microinjection procedure appeared to be not entirely reproducible. Then we proceed with observation of the developmental stage by looking at the nuclear structure in the cortical array by exciting VIS fluorescence of nuclear histones, recorded by the EMCCD camera $(1024 \times 1024$ pixels, $13 \mu \mathrm{m}$ pixel resolution, iXon DU-888E; Andor Technology, Belfast, UK). By observing VIS nuclear fluorescence we obtain at each timepoint exact knowledge about the developmental state and the thermodynamic fate of the embryo. We decided to first expose embryos to NIR Ti:Sapph laser light and afterwards to the $488 \mathrm{~nm}$ laser, because by exposing the embryo at first with a wavelength much larger $(770 \mathrm{~nm})$ than the absorption peak of EGFP $(488 \mathrm{~nm})$, we avoid premature photobleaching of nuclear VIS fluorescence. Another reason 
for using the lasers in this sequence was that the behavior of CNTs suspension in Drosophila embryos is by far more critical than the state of embryos themselves. In many cases CNT concentration in the embryo was too low (regardless of the embryonic type) or a prevalent amount of CNTs appeared in form of clusters. In these pathological cases we started over, using the NIR transillumination diode lamp to select another embryo.

After being convinced the embryo on hand comprises a reasonable amount of CNTS and is in an early syncytial stage, we turn off the NIR transillumination and further use the $488 \mathrm{~nm}$ laser to set the focal plane ( $x$-y-plane) to have it intersect the cortical layer. Now that we have set the basis for the actual recording including, i. e. the simultaneous observation of NIR CNT fluorescence yield and VIS]EGFP fluorescence, we can start with the acquisition of images.

In this work, we used multimodal imaging, i. e. two distinct imaging channels to simultaneously observe intracellular dynamics throughout successive developmental stages. In that course, we subjected the embryos to the NIR Ti:Sapphlaser for $30 \mathrm{~min}$, exciting CNTs in one embryo and started acquisition by the InGaAs CMOS camera (XEVA-FPA-1.7-320; Xenics, Leuven, Belgium) to record CNT fluorescence. For this purpose we used the software Xeneth which is especially designed for controlling the InGaAs CMOS camera we used in our experiments. Directly after starting acquiring CNT signals, we began to record signals from His-EGFP with an EMCCD camera. To control the EMCCD camera for the imaging of His-EGFP during multimodal imaging, we used the open-source software Micro-Manager [144, 160]. Table 6.1 provides an overview of the parameters used during the experiments. For controlling the shutter directly behind the $488 \mathrm{~nm}$ laser during the actual image acquisition, we connected the "fire" plug at the EMCCD camera to the shutter box which in turn was connected to the shutter.

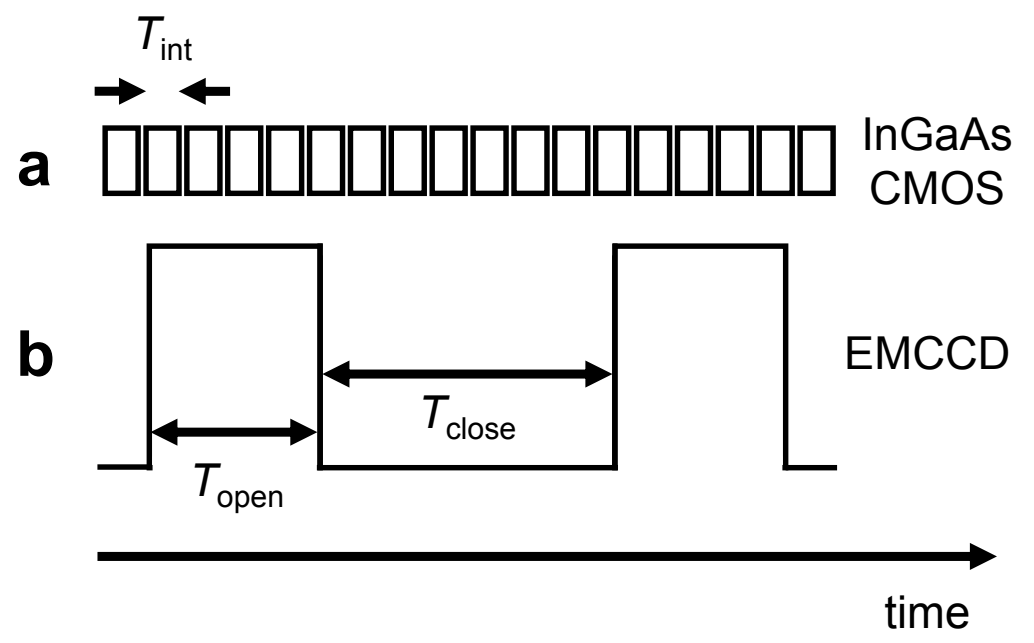

Figure 6.1. Generic protocol for simultaneous VIS NIR imaging. Rectangles indicate individual images recorded by the InGaAs CMOS camera (a). Integration time $T_{\text {int }}$ of the InGaAs CMOS camera amounts to $100 \mathrm{~ms}$. $T_{\text {open }}=1 \mathrm{~s}$ describes the opening time for the EMCCD camera (b). The shutter of the EMCCD camera closes for $T_{\text {close }}=19 \mathrm{~s}$ during single-nanoparticle tracking experiments and for $T_{\text {close }}=4 \mathrm{~s}$ during imaging of the cytoplasmic flow (b). 
The EMCCD camera triggers the mechanical shutter (Fig. 6.1) via Micro-Manager according to the parameter set given in table 6.1. In case of comparative singlenanoparticle experiments (Chap. 6.3), the mechanical shutter cloes for $19 \mathrm{~s}$. During imaging of cytoplasmic flow (Chap. 6.4), the mechanical shutter closes for $4 \mathrm{~s}$ (Tab. 6.1).

Table 6.1. Parameters fed into Micro-Manager for imaging with EMCCD camera. We use an exposure time of $1 \mathrm{~s}$ and record 3 frames per min, i. e. 90 frames over a total measurement interval of $30 \mathrm{~min}$ for comparative single-nanoparticle experiments. For mesoscopic bulk studies, we opened the mechanical shutter behind the $488 \mathrm{~nm}$ laser every $5 \mathrm{~s}$ (360 times in total).

\begin{tabular}{lll}
\hline Parameter & Value (Chap. 6.3) & Value (Chap. 6.4) \\
exposure time & $1000 \mathrm{~ms}$ & $1000 \mathrm{~ms}$ \\
time points & 90 & 360 \\
interval & $20 \mathrm{~s}$ & $5 \mathrm{~s}$
\end{tabular}

In both cases (single-nanoparticle tracking and bulk studies), the shutter remained opened for $1 \mathrm{~s}$ (Tab. 6.1, exposure time) while exposing the embryo continuously to $770 \mathrm{~nm}$ excitation light from the Ti:Sapph laser for $30 \mathrm{~min}$.

Through this process we obtained a generic protocol for imaging living Drosophila embryos throughout a total experimentation time of 30 min allowing for simultaneous observation of VIS fluorescence of nuclei and NIR fluoescence of CNTs over successive mitotic phases (M phases) and interphases (Fig. 6.1).

\section{Challenges during imaging}

During imaging we faced some challenges, which we briefly want to outline in the following. For imaging CNTs in living Drosophila embryos as basis for singlenanoparticle tracking, a good dispersion state of CNTs is vital. Interestingly, in some cases CNTs predominantly accumulated around nuclei in the imaging plane. Since this occurance happened throughout both embryo types (His-EGFP and His-EGFP/kin-5-Halo, we speculate that this is a generic property of CNT-cortex interaction arising from an accumulation of mechanical entanglement of CNTs with cytoskeletal elements in vicinity of nuclei (Fig. 6.2). 
A

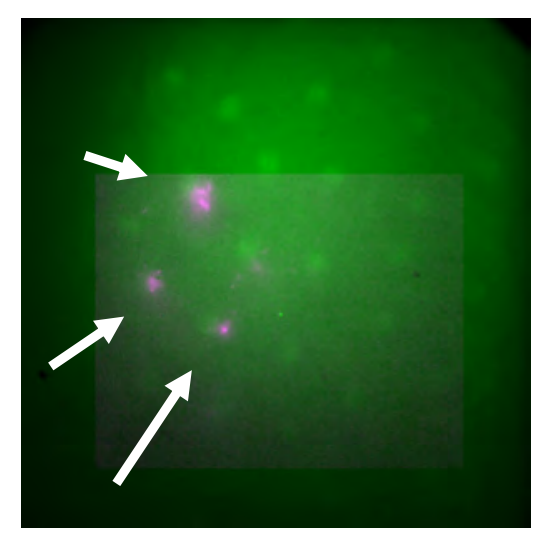

C

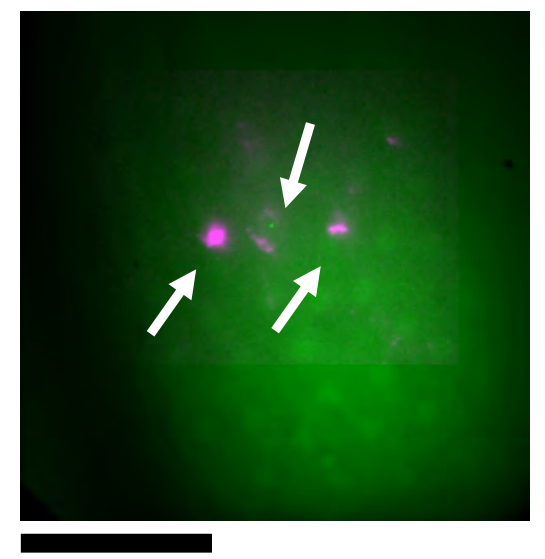

B

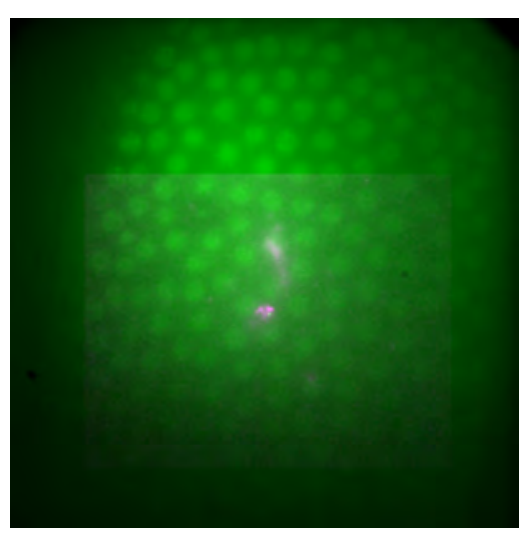

$\mathbf{D}$

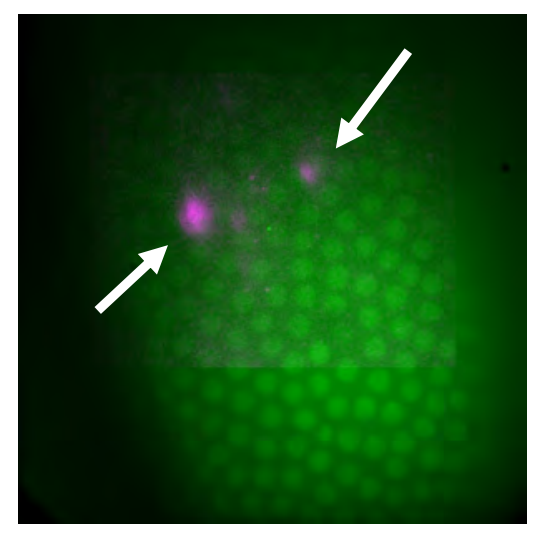

$50 \mu \mathrm{m}$

Figure 6.2. Assembly of CNTs (magenta, indicated by white arrows) in the vicinity of nuclei (green) in both embryos expressing His-EGFP (A), (B) and in embryos expressing His-EGFP/kin-5-Halo (C), (D). Panels (A) and (C) show the embryos in early interphases, panels (B) and (D) in later interphases.

Moreover, we found that most CNTs assembled directly below the nuclei close to the yolk. This made it difficult to detect particular CNTs in the cortex. We speculate, that CNTs entangled in cytoskeletal filaments, such as astral microtubules pointing towards the yolk. We also observed the raise in contrast with increasing age due to depletion of the fluorescing yolk cell (Fig. 6.5). This shortened the time window for high-contrast imaging His-EGFP, depending on the particular embryo under investigation, from interphase 11 or 12 to late interphase 13. After interphase 14, cellularization begins during which nuclei are separated and suppresses microtuble mediated internuclear correlations. During imaging the influence of the observer on the behavior of the CNTs was negligible. Furthermore, the yield of fluorescent CNTs in the embryos differed considerably from one embryo to another. 


\subsubsection{Image registration}

When imaging with two cameras, it is important to ensure that both cameras can see the same picture at the same time. Tracking experiments in Drosophila embryos require an exact alignment of the FOV in all three spatial directions. Furthermore, it requires an exposure time adapted to the fluorescence properties of the respective fluorophores (His-EGFP and CNTs). However, the two cameras we used display photosensitivity in different wavelength regimes. The EMCCD camera records fluorescence emission in the VIS while the InGaAs CMOS camera records in the NIR fluorescence of CNTs,

In order to perform both the temporal and the spatial calibration, we apply the so-called image registration spatially and temporally. We describe this procedure in the following.

\section{Spatial alignment}

In order to obtain the same FOV in both channels, the three spatial directions must be aligned and calibrated. For this we use red fluorescent beads (FluoroMax ${ }^{\mathrm{TM}}, \mathrm{R}$ 600, $0.6 \mu \mathrm{m}$; Thermo Fisher Scientific Life Technologies GmbH, Darmstadt, Germany), which have been shown to be visible in both channels. These beads provide an unambiguous pattern in the FOV of each camera. We use this bead pattern as the basis for spatially superimposing the two image sections.

In order to set parfocality in both imaging channels, we align dichroic mirror $\mathrm{D}_{6}^{*}$ in the detection system of the imaging setup (Fig. 4.1 and Fig. 4.2) by focussing an object being visible in both channels. We put $5 \mu \mathrm{l}$ of red-fluorescent beads in water (1:50) on a coverslip of a thickness of $0.17 \mathrm{~mm}$ (microscope slides, Menzel-Gläser, $22 \times 40 \mathrm{~mm}$; VWR ${ }^{\circledR}$ International GmbH, Dresden Germany). Then we wait about $30 \mathrm{~min}$ for the solvent to evaporate until only a thin layer of beads is left on the coverslip (Fig. 6.3 and Fig. 6.4). We thus find parfocality by focussing a pattern of dried-out beads in both imaging channels. The advantage of using beads is, that they have a defined diameter (here the bead diameter is $0.6 \mu \mathrm{m}$ ). Additionally, beads give a pattern which later helps merging both imaging channels during image registration.

A

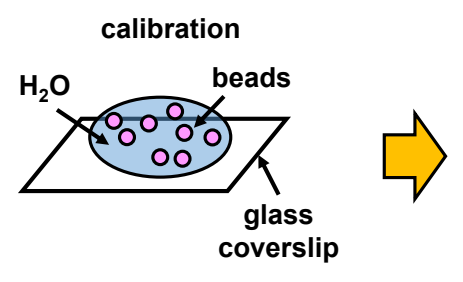

B

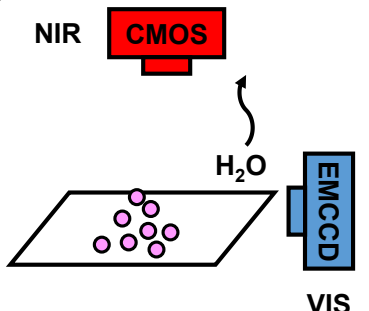

C

image registration

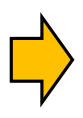

Figure 6.3. Schematic of the calibration for parfocality for the multimodal VISINIR imaging. A. Redfluorescent beads in watery solution (1:20) are placed on a glass coverslip. B. The watery solvent evaporates, leving behind a pattern of dry beads on the glass coverslip. C. Registration of images by using bead pattern. 

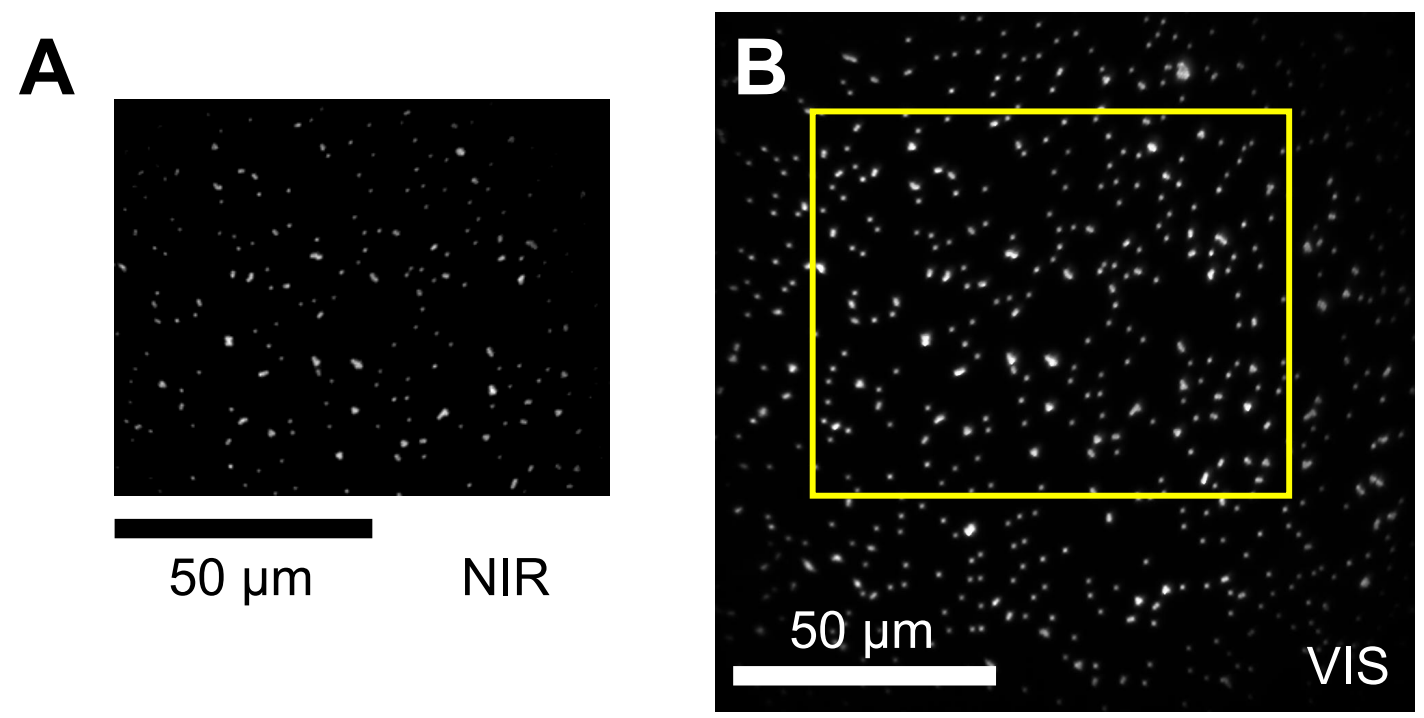

Figure 6.4. Bead pattern for FOV calibration for VISTIR imaging In course of image registration, a bead pattern is detected in both the $\mathrm{NIR}(\mathbf{A})$ and the VIS channel (B). Bead patterns are superimposed at points which enclose the same FOV For that reason, beads are focussed in both channels sharing one focal plane. Positioning of the bead pattern in the focal plane during post-processing allows to extract coordinates to place the smaller frame (NIR A) into the larger one (VIS B), as indicated by the yellow frame in $(\mathbf{B})$.

Compared to CNTs, we could observe a fast bleaching of beads in the NIR channel. Thus, in order to obtain a high contrast, imaging of beads has to happen in less than 30 sec. The beads are freshly prepared before each measurement session.

By the procedure described above we achieve a calibration of both field of views (FOVs), which makes it possible to monitor both imaging channels in a shared focal plane.

In this work, we use morphological changes of individual nuclei in the nuclear array during embryonal development for spatio-temporal orientation. Nuclei in Drosophila embryos have diameters of about $10 \mu \mathrm{m}$ [30] (Fig. 2.2) and host genetic information in form of DNA [43]. During our experiments, we observe fluorescence EGFP; 15, 161, 162] located at histones over successive nuclear division cycles (Fig. 6.5 delivering information about tissue depth and developmental stage. Here, we use EGFP absorbing light at $489 \mathrm{~nm}$ (blue) [14] and emitting at $509 \mathrm{~nm}$ (green) [14, 16] with a fluorescence lifetime in the order of 1 ns [14]. Histone co-localization with chromosomes [163] makes histones ideal tagging sites for obtaining spatio-temporal orientation in Drosophila embryos. Hence, in this work, all Drosophila embryos we use express green fluorescent protein at histones, providing direct information about the developmental stage. As figure 6.5 demonstrates, during interphase, nuclei appear as roundish objects (Fig. 6.6, A, C, E, F), while during M phase, chromosome separation becomes visible (Fig. 6.6, B, D). 


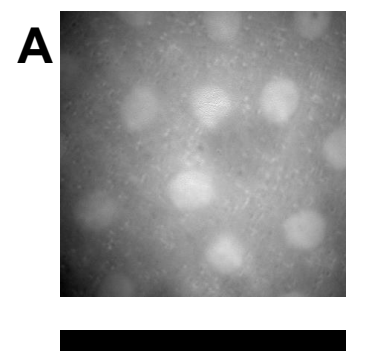

$50 \mu \mathrm{m}$
B
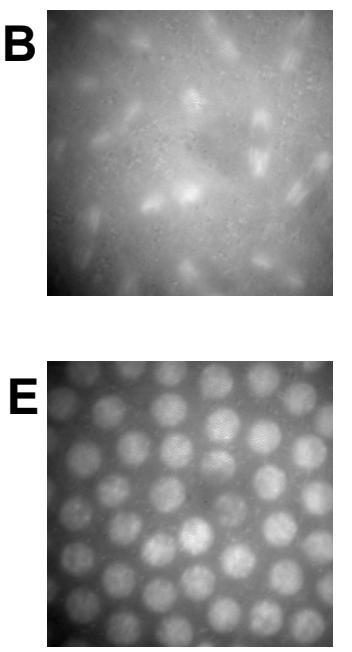
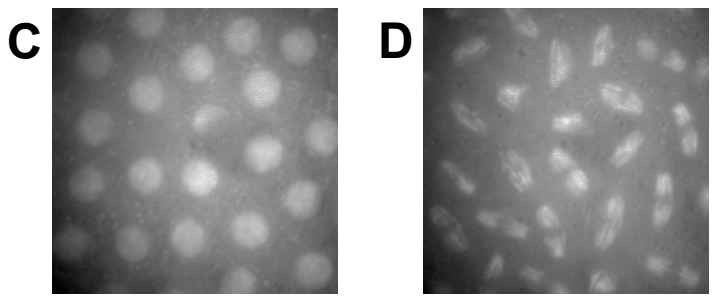

$\mathbf{F}$

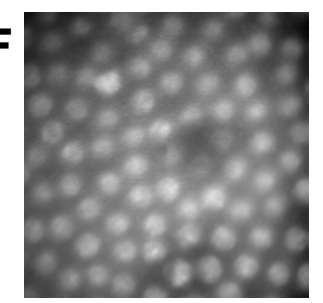

G

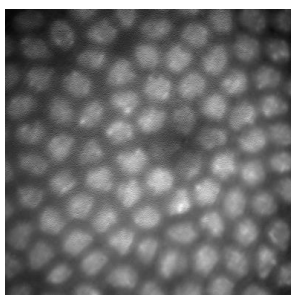

Figure 6.5. Recording of His-EGFP in several nuclear division cycles of Drosophila. Nuclei in Drosophila embryos during interphase 11 (A), M phase $11 / 12$ (B), interphase 12 (C), M phase 12/13 (D), interphase $13(\mathbf{E})$, interphase $14(\mathbf{F})$ and during beginning cellularization $(\mathbf{G})$.

When recording CNTs with the InGaAs CMOS chip, pixel size amounts to $30 \mu \mathrm{m}$. The acquisition of VIS EGFP fluorescence of nuclei is recorded with an EMCCD chip with pixel size of $13 \mu \mathrm{m}$ (Fig. 6.6). This means, during spatial image registration we have to deal with different pixel sizes of the detector chips. It is reasonable to always fit the coarse grid with the larger pixel size on the fine grid with the smaller pixel size. In case of the experiments performed in this work, this means that we fit the NIR channel with its larger pixels and with its smaller [FOV into the grid of the VIS channel, having smaller pixel size and a larger FOV.
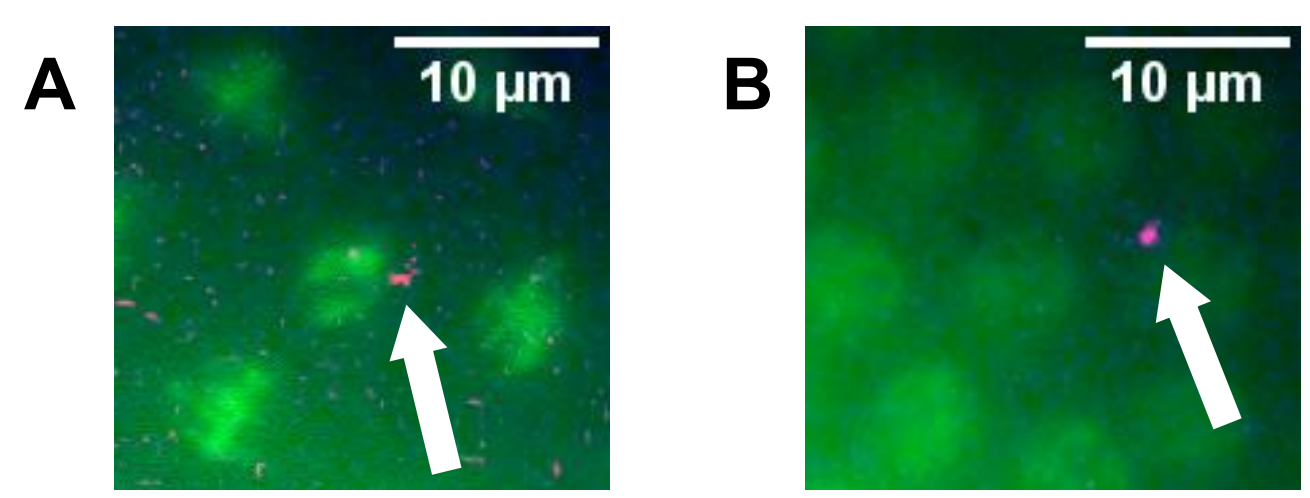

Figure 6.6. Registered images of nuclei in Drosophila embryos and NIR fluorescent CNTS The panels show CNTs NIR fluorescence signals (magenta, indicated by white arrows) and nuclei (green) during two distinct developmental phases of the Drosophila embryo: A. M phase and B. interphase. Both images show the two channels (NIR and $\mathrm{VIS}$ ) being registered as described above.

Consequently, we obtain a registered image with the same dimensions as the EMCCD chip $(1024 \times 1024)$. By upscaling the $\mathrm{NIR}$ image by a factor of $30 / 13$ the pixels in the NIR image are interpolated to fit into the fine grid of the VIS image. 


\section{Time series of images}

In the course of the experiments, we were interested in the temporal evolution of CNT dynamics in the Drosophila embryos which we recorded for a total period of 30 min. When using two imaging channels operating in mutually exclusive wavelength detection regimes for recording intracellular fluorescence, it is important to know about the spatio-temporal orientation. Thus, we use image registration, meaning superimposing these two channels (NIR and VIS) onto one another.

As the protocol (Chap. 6.1.1) shows, we use different illumination times to account for the different fluorescence properties of CNTs and EGFP. Therefore, the images of the individual channels must be superimposed in such a way that the synchronization of both channels is reproduced during imaging.

In our studies, we have in average acquired 10 frames per second in the NIR channel, while the VIS was sampled at lower frequency $(0.05 \mathrm{~Hz}$ and $0.2 \mathrm{~Hz})$.

The total number of frames in the NIR channel during the experimental time of 30 min amounts to

$$
\begin{aligned}
\frac{\text { total number of frames }(\mathrm{NIR} \text { channel })}{30 \mathrm{~min} \text { experimental time }} & =30 \mathrm{~min} \cdot 60 \frac{\mathrm{s}}{\mathrm{min}} \cdot 10 \frac{\text { frames }}{\mathrm{s}} \\
& =18000 \text { frames. }
\end{aligned}
$$

Accordingly, under appropriate choice of setting parameters, the total frame number in the VIS channel acquired in single-nanoparticle experiments (Chap. 6.3) amounts to 90 frames / $30 \mathrm{~min}(0.05 \mathrm{~Hz})$ :

$$
\frac{\text { total number of frames (VIS channel) }}{30 \mathrm{~min} \text { experimental time }}=30 \mathrm{~min} \cdot 3 \frac{\text { frames }}{\min }=90 \text { frames. }
$$

In experiments probing mesoscopic bulk dynamics, we acquire five frames per second in the VIS (Chap. 6.4). In this case, the we record 360 frames / $30 \mathrm{~min}(0.2 \mathrm{~Hz})$ :

$$
\frac{\text { total number of frames (VIS channel) }}{30 \text { min experimental time }}=30 \mathrm{~min} \cdot 12 \frac{\text { frames }}{\min }=360 \text { frames. }
$$

It is now important to map the NIR and the VIS channel. In this chapter, we show how the above-mentioned channels are combined. In the following, we describe the registration procedure for the single-nanoparticle experiments (Chap. 6.3.1). The registration procedure for bulk studies (Chap. 6.4) follows analogously (Eq. 6.3). For image registration, we superimpose images in portions of 100 and 200. Here, $\left\{v_{j}\right\}_{j=\{1,2, . .90\}}$ defines the set of images of the VIS channel obtained from the EMCCD camera and $\left.\left\{n_{k}\right\}_{k=\{1,2, . .} 18000\right\}$ is set of the simultaneously recorded images of the NIR channel obtained from the \nGaAs CMOS camera. We define the set of registered images $r$ as the ordered set $(v, n)$. The tuple $r_{k}$ is defined by the relation

$$
r_{k}:=\left(v_{j}, n_{k}\right)
$$


Here, the registered images $r$ and the images $n$ of the NIR channel share the same indices $k$ which runs from the first NIR image.

$$
\begin{aligned}
\mathbf{r}^{j<2} & =\left(\left\{r_{k}\right\}_{k=\{1, . ., 100\}}\right) \\
& =\left(r_{1}, r_{2}, . ., r_{100}\right) \\
& =\left(\left[\begin{array}{c}
v_{1} \\
n_{1}
\end{array}\right],\left[\begin{array}{c}
v_{1} \\
n_{2}
\end{array}\right], . .,\left[\begin{array}{c}
v_{1} \\
n_{100}
\end{array}\right]\right)
\end{aligned}
$$

For all the VIS images, beginning from the second one, the registration process looks like the following:

$$
\begin{aligned}
\mathbf{r}^{j \geqslant 2} & =\left(r_{k}\right)_{k=\left\{101, . ., k_{\max }\right\}} \\
& =\left(r_{101}, . ., r_{300} ; r_{301}, . ., r_{500} ; r_{17701}, . ., r_{17900}\right) \\
& =\left(\left[\begin{array}{c}
v_{2} \\
n_{101}
\end{array}\right], . .,\left[\begin{array}{c}
v_{2} \\
n_{300}
\end{array}\right] ;\left[\begin{array}{c}
v_{3} \\
n_{301}
\end{array}\right], . .,\left[\begin{array}{c}
v_{3} \\
n_{500}
\end{array}\right] ; . . ;\left[\begin{array}{c}
v_{90} \\
n_{17701}
\end{array}\right], . .,\left[\begin{array}{c}
v_{90} \\
n_{17900}
\end{array}\right]\right)
\end{aligned}
$$

In (Eq. 6.6), semicola denote the change of a VIS image. The sets of registered images defined in (Eq. 6.6) and (Eq. 6.7), we then combine in a resulting ordered set of registered images $\mathbf{r}$ :

$$
\begin{aligned}
\mathbf{r} & =\left(\mathbf{r}^{j<2} ; \mathbf{r}^{j \geqslant 2}\right) \\
& =\left(r_{1}, r_{2}, . ., r_{100} ; r_{101}, r_{102}, . ., r_{300} ; . . . r_{17701}, r_{17702}, . ., r_{17900}\right)
\end{aligned}
$$

In (Eq. 6.7), again semicola divide sets of NIR images $n_{k}$ which share one common VIS image $v_{j}$. The following is an example of how the registration protocol defined in equations (Eq. 6.4) and (Eq. 6.5) can be implemented in MATLAB:

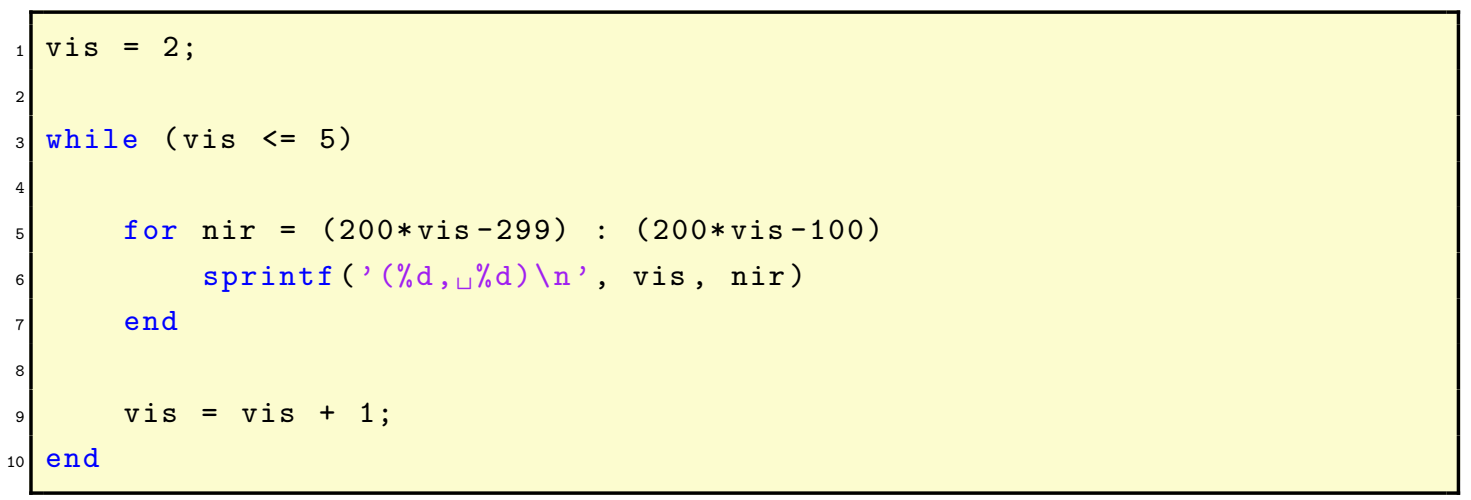

Figure 6.7 shows an example for stacking image sequences during overall recording time of $30 \mathrm{~min}$. 


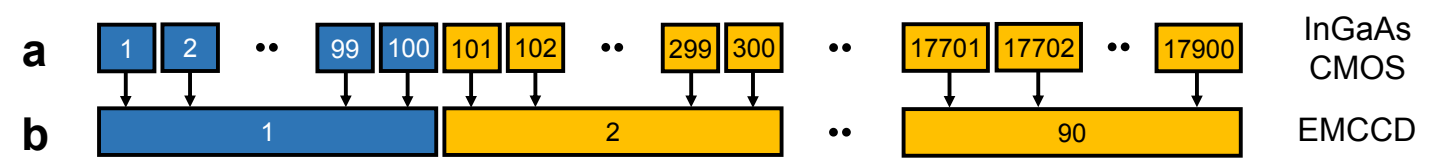

Figure 6.7. Exemplary protocol of the registration of the image sequences during the multimodal[VIS.NIR] image sequences. For the temporal superposition, images from the InGaAs[CMOS camera (a) are grouped and binned into one respective frame recorded by the EMCCD camera (b).

\section{Kymographs}

A common way in biology to represent spatio-temporal evolution of a system, are kymographs. Kymographs portray graphically the motile time-evolution of a tracer particle such as a CNT. Typically, kymographs are represented as $t$-x-diagram with one axis representing time while the other plots a spatial direction. Here, we present kymographs of individual CNTs in both His-EGFP and His-EGFP/kin-5-Halo embryos (Fig. 6.8). Kymographs provide a first qualitative overview, whether the motile behavior of CNTs differs in these two different embryo types. In case of sucessful linking of CNTs to kinesin-5 molecules, we expect a majority of found CNT trajectories in His-EGFP/kin-5-Halo embryos to display more directed motion and less random movements as His-EGFP embryos. Hence, a corresponding kymograph for His-EGFP/kin-5-Halo embryos, in contrast to His-EGFP should predominantly display straight lines. However, we found a comparable amount of movements in His-EGFP embryos to show also directed behavior (Fig. 6.8). The crowded nature of the intracellular space of Drosophila leaves possibilties for unspecific binding to other agents inside the cell providing directed transoport. For instance, we speculate that directed movements in His-EGFP embryos arise from attachment of CNTs to vesicles or other agents. Using kymographs is a good method for a first impression on the data. However, full and exact characterization requires quantitative methods like single-particle tracking (Chap. 6.2). 


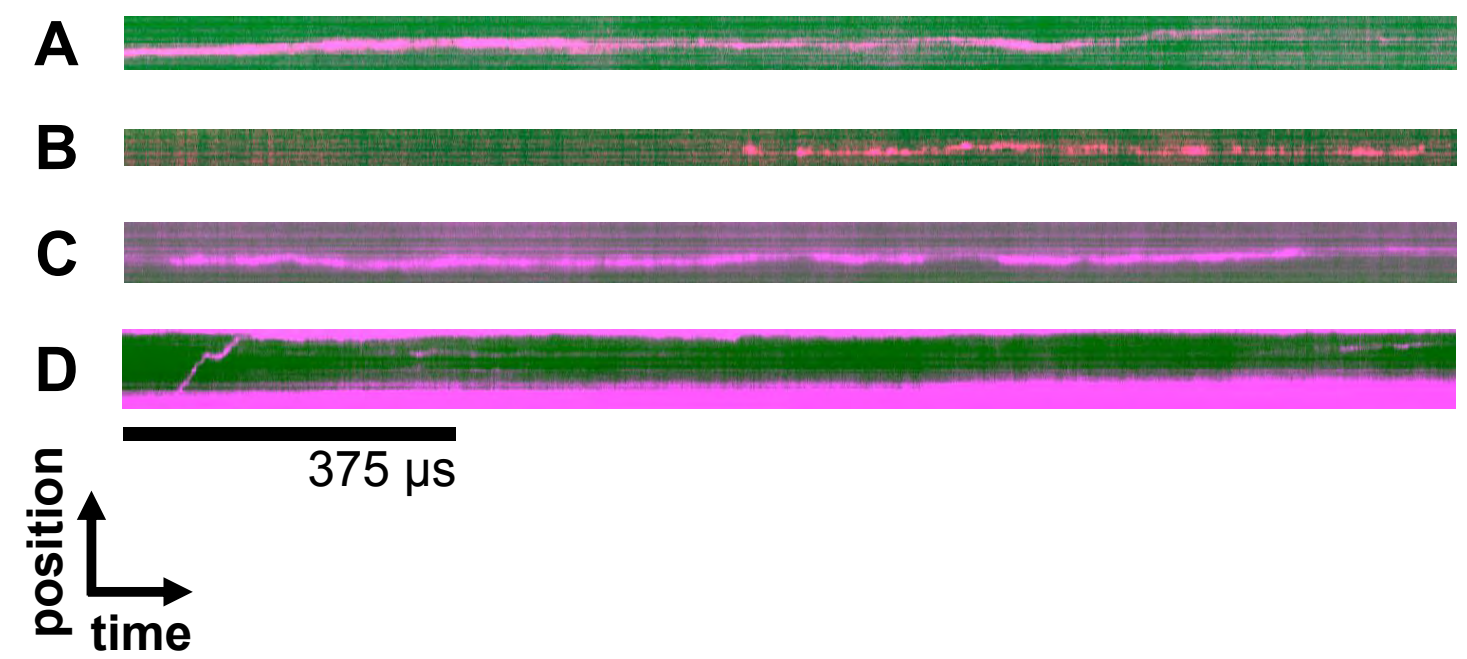

Figure 6.8. Exemplary kymographs of individual CNTs in the cortex of a Drosophila embryo. Kymographs obtained by multimodal imaging of live embryos expressing His-EGFP (A), (B) and embryos expressing His-EGFP/kin-5-Halo (C), (D). Magenta traces show CNT signals. D. Anecdote of a linear movement of a CNT in a His-EGFP/kin-5-Halo embryo with a velocity of about $9 \mu \mathrm{m} / \mathrm{s}$.

Using kymographs we find anecdotes of functionalized CNTs displaying directed motility in Drosophila embryos. However, in many cases these traces show velocities surpassing those of kinesin-5 by two orders of magnitude [164]. Moreover, these directed traces can also be observed in His-EGFP embryos which do not have a HaloTag ${ }^{\circledR}$ binding site.

\subsection{Tracking of individual carbon nanotubes in living Drosophila embryos}

Tracking of individual CNTs inside the Drosophila embryo is crucial to gain quantitative and local information about intracellular dynamics. Tracks of single CNT molecules deliver information about quantities characterizing dynamical behavior of CNTs such as mean squared displacements (MSDs) or velocity cross-correlations. Here, we use tracking as a basis to compare the motile behavior of individual functionalized CNTs in two embryo types (His-EGFP and His-EGFP/kin-5-Halo) (Chap. 6.3). The tracking parameters were the basis for the later mean squared displacement (MSD) analyses (Chap. 6.3.1) and velocity cross-correlations (Chap. 6.3.4). For tracking, we use the open-source tracking software TrackNTrace [165. TrackNTrace is well documented and allows for user specific input of parameters, necessary for tracking individual particles. In general, tracking of individual molecules requires a delicate choice of parameters. On the one hand one has to make sure to not track in the noise, on the other hand minimization of dectecting false negatives has to be ensured. TrackNTrace provides input options for nearest neighbor tracking. These options include five parameters which can be chosen freely. A full set of parameters is compulsory for being able to start the tracking process. In the following we present our choices of these parameters and reason our decisions. Table 6.2 provides an 
overview on the following five key parameters used for single-nanoparticle tracking. First, we set the value of minimal trajectory length (minTrajLength) to 10. This means, we consider only trajectories having a presence for at least 10 frames or, equivalently, $1 \mathrm{~s}$. We did this because this vaule allows us to detect the most CNTs possible, in contrast to a higher value. The second parameter allows input of the maximal allowed linking distance of two locations (maxTrackRadius). With this parameter, we define that in case of one candidate to track has been found, we only allow connecting points in the vicinity of two pixels around this candidate. By choosing a low value for maxTrackRadius, we aim at reducing chances for detecting false positives from the noise. Because CNTs are intermittency-free, we decided to not allow blinking signals to be included in the tracking process by zeroizing the maximal time gap between between two frames (maxFrameGap). During tracking, it turned out that trajectories of a particular CNTs could not persistently be tracked but instead moved out of the FOV. Hence, we could argue, that, albeit CNTs do not blink, their disappearing and subsequent reappearance can be considered as a form of blinking. However, we decided to not consider this assumption and considered it as less expedient, since during measurments in living embryos, CNTs move also inside the focal plane. Since the minimum segment length before frame gap closure (minSegLength) is dependent on maxFrameGap and since we chose this value to be 0 frames, we kept for simplicity the default parameter of minSegLength, which was set to 1 frame. We chose the maximal pixel distance before closing the gap between candidate pixels (maxGapRadius) to be 6 pixels to consider trajectory segments from particular CNTs which possibly intersect the focal plane at different locations and thus, to aim at stichting together tracks of the same CNT candidate.

Table 6.2. Parameters used for particle tracking with TrackNTrace.

\begin{tabular}{lll}
\hline parameter & Parameter name in TrackNTrace & Value \\
\hline minimal trajectory length minTrajLength & 10 \\
maximal track radius & maxTrackRadius & 2 \\
maximal frame gap & maxFrameGap & 0 \\
minimal segment length & minSegLength & 1 \\
maximal gap radius & maxGapRadius & 6
\end{tabular}

These parameters are the results of careful observation of different settings. After testing multiple parameter sets, these parameters turned out to be the most promising. In other contexts (different cameras, CNTs, embryos etc.) and under different conditions, the parameters may vary.

Even when using an appropriate paramter set (Tab. 6.2), in many cases, detected trajectories of individual CNTs appeared to be broken up into multiple ones, resulting in shortend trajectories. We used custom-written software to further process the data obtained by tracking with TrackNTrace. 

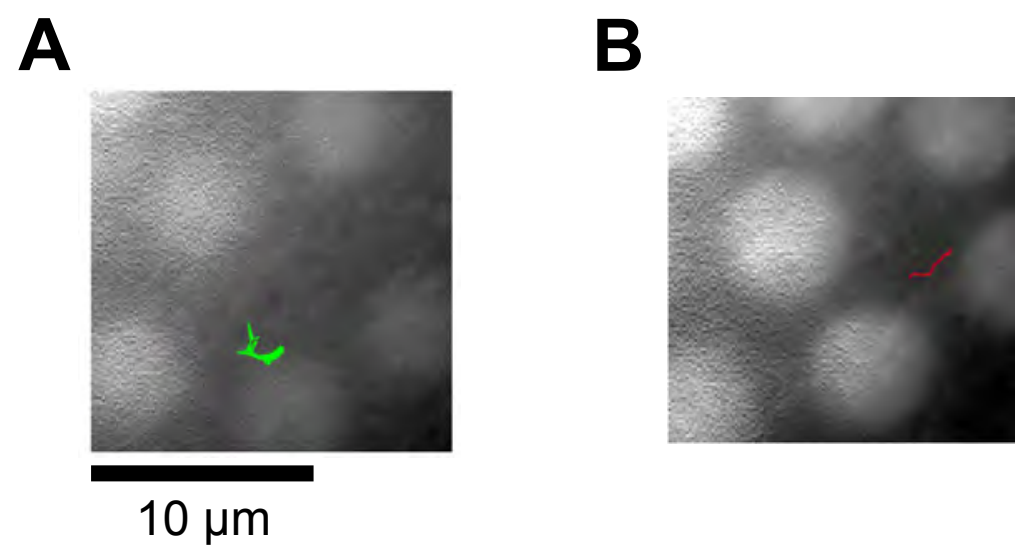

Figure 6.9. Trajectories of CNTs in Drosophila embryos showing brownian motion (A, green) and directed motion (B, red). The traces were obtained from tracking with TrackNTrace [165]).

Throughout our experiments, we found that histograms for the two embryo types (His-EGFP and His-EGFP/kin-5-Halo) qualitatively displayed similar behavior, independent of the embryo type (Fig. 6.10). Data in (Fig. 6.10, A) shows the counts of tracks in one single embryo expressing His-EGFP, whereas (Fig. 6.10, B) depicts an analogous diagram for an embryo expressing His-EGFP/kin-5-Halo, In both fly crosses, lifetime of CNT trajectories was in most cases lower than $1 \mathrm{~s}$, while only few tracks appeared for more than 1 min in the FOV. 
A
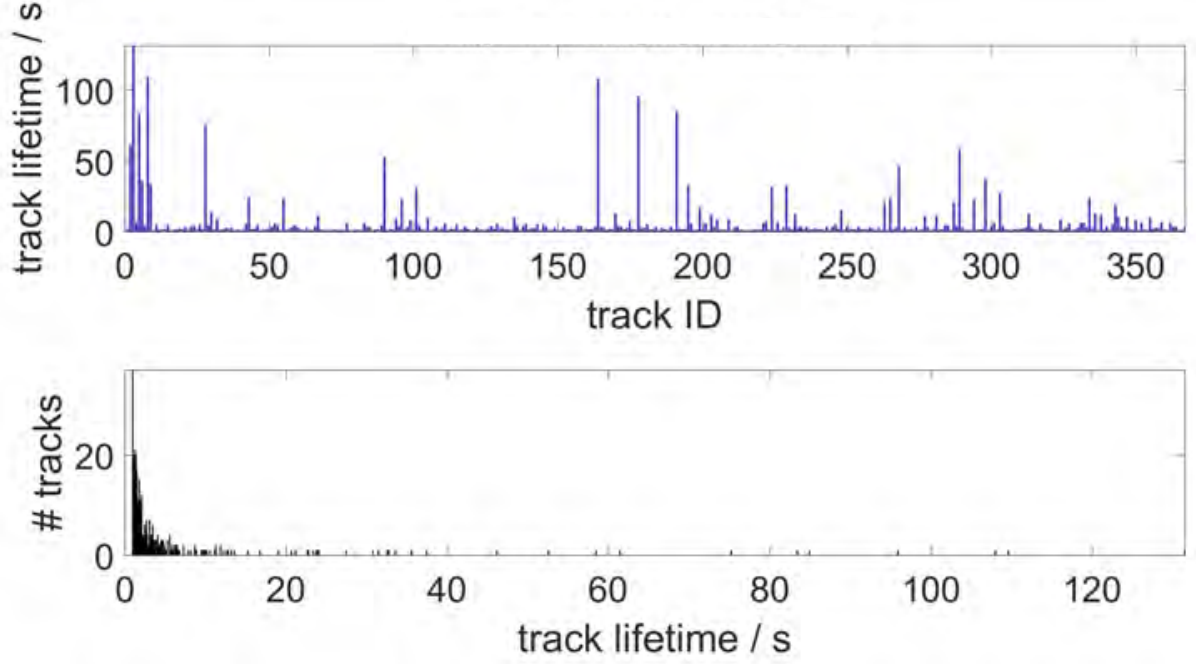

B
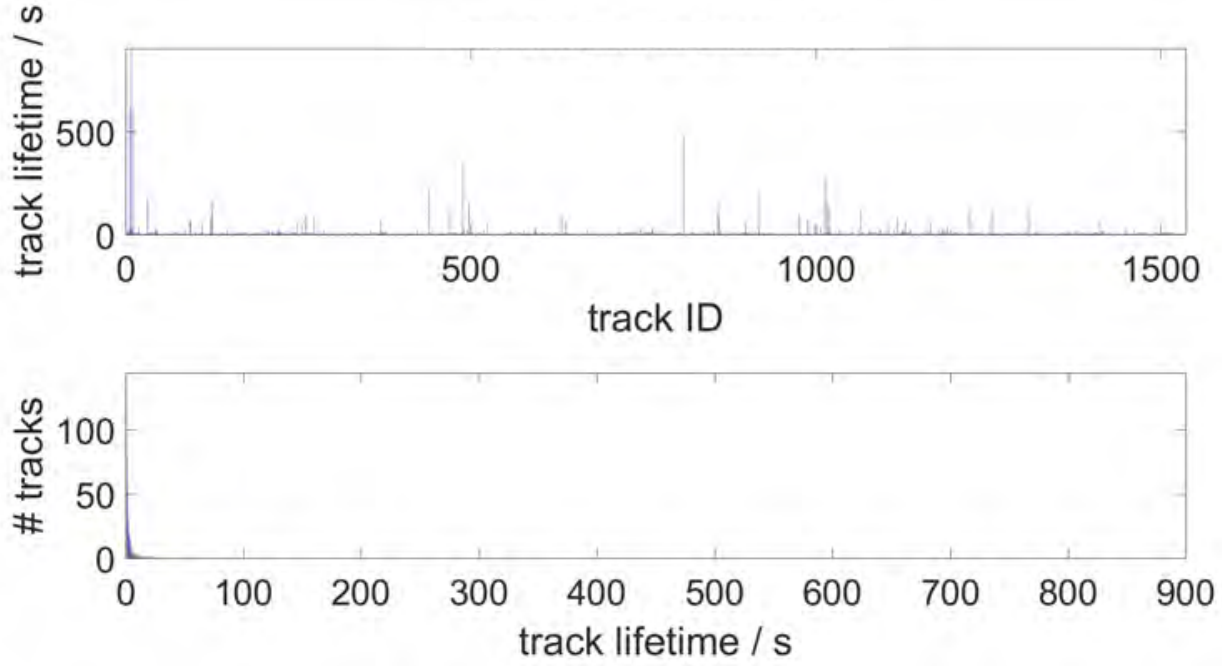

Figure 6.10. Statistics of track lifetimes of individual CNTs in embryos His-EGFP and His-EGFP/kin-5-Halo). A. The upper panel shows the lifetime of individual CNT trajectories as counts vs. track ID. The lower panel depicts the histogram of the lifetime. Both panels show individual tracks of the same individual CNTs in embryos expressing His-EGFP B. The upper panel shows the lifetime of individual CNT trajectories as counts vs. track ID. The lower panel depicts the histogram of the lifetime. Both panels show individual tracks of the same individual CNTs in embryos expressing His-EGFP/kin-5-Halo. 
A

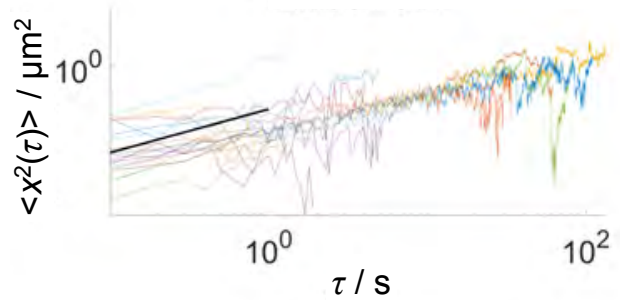

C

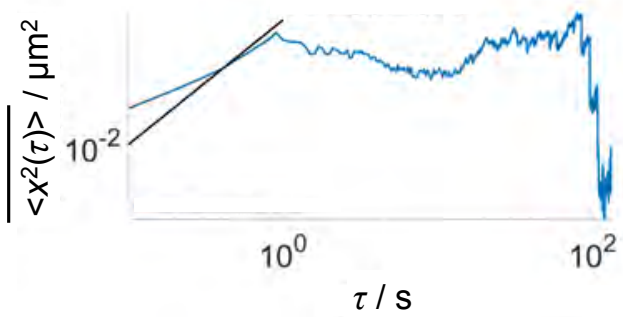

E

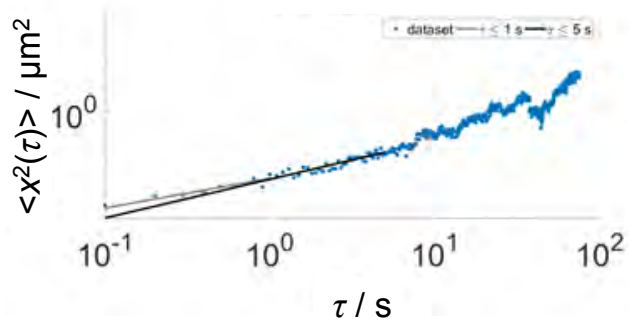

B

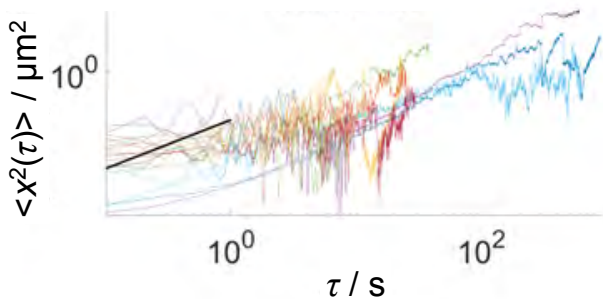

D

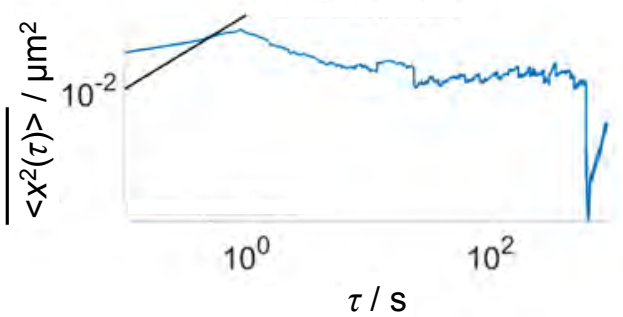

$\mathbf{F}$

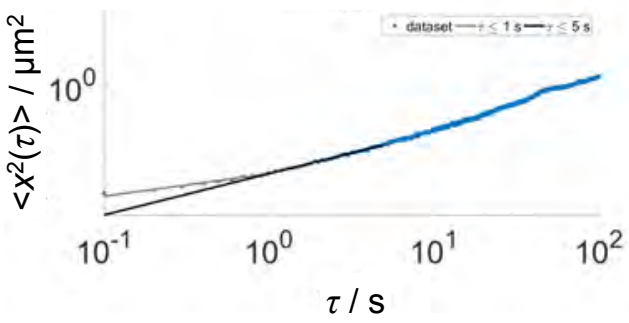

Figure 6.11. MSDs of CNTs in embryos His-EGFP vs. His-EGFP/kin-5-Halo. (A) Array of measured MSD curves of individual CNTS in an embryo expressing His-EGFP and an embryo expressing His-EGFP/kin-5-Halo (B). Panels (A) and (B) show 20 arbitrarily selected measured MSDs of individual CNTs in living embryos. Averaged MSDs in an embryo expressing His-EGFP (C) and in an embryo expressing His-EGFP/kin-5-Halo (D). In panels (A) to (D), the black line shows the MSD slope $\equiv 1$ for comparison. Exemplary LMSD of an individual CNT in an embryo expressing His-EGFP (E) and in an embryo expressing His-EGFP/kin-5-Halo (F). In panels (E) and (F), measured data is shown as blue dots, the linear fit of the measured MSD for the short time interval $\left(\tau_{1} \in[0 ; 1] \mathrm{s}\right)$ is shown as gray line and the linear fit of the measured MSD for the long time fit interval $\left(\tau_{2} \in[0 ; 5] \mathrm{s}\right)$ is depicted as black line.

\subsection{Single-nanoparticle dynamics of fluorescent carbon nanotubes in living Drosophila embryos}

\subsubsection{Mean squared displacements}

The MSD is a measure for the area $\left\langle x^{2}(\tau)\right\rangle$ an object passes during a time step $\tau$. MSD analyses are commonly used in biology as a measure to categorize different types of motility based on the diffusive behavior of tracer particles in a medium [6, 166, 167]. In this work we use this widely accepted idea to obtain information about the dynamical behavior of fluorescent CNTs in the Drosophila embryos. From the behavior of a particle of interest performing Brownian motion, we can also gain 
information about the diffusivity $D$ the particle. A 2D MSD with diffusivity $D$ yields power law behavior for lag time $\tau$ with anomaly parameter $\alpha$ [168]:

$$
\left\langle x^{2}(\tau)\right\rangle=4 D \tau^{\alpha} .
$$

In this chapter, we focus on the analysis of the anomaly parameters $\alpha$ of both, His-EGFP and His-EGFP/kin-5-Halo embryos. Data obtained from single-nanoparticle tracking (Chap. 6.2) was the starting point of the studies presented in this chapter. Typically, motor movements along cytoskeletal filmaments such as kinesin-1 motors processing along microtubules display directed motility [6]. Here we want to apply the method of MSD analysis as a tool for systematic characterization of NIR fluroescent CNTs in embryos expressing His-EGFP and embryos expressing His-EGFP/kin-5-Halo, By presenting MSDs of individual CNT molecules, obtained from single-nanoparticle tracking of functionalized CNTs in living Drosophila embryos (Fig. 6.11), we prove successful application of CNTs as fluorescent probes in Drosophila embryos during continuously exposing embryos to NIR laser light for 30 min. We were able to extract trajectories (Fig. 6.11, A and C) which can be used for further analysis of the anomaly parameter $\alpha$. Basis for MSD analyses was singleparticle tracking (Chap. 6.2) from which we obtained the individual trajectories used in the further analyses (Chap. 6.2). For each of these CNT trajectories in the cortical region of both embryo types during $30 \mathrm{~min}$ acquisition time (Chap. 6.1.1) we computed the corresponding set of MSDs (Fig. 6.11, A). For better overview we did not depict all the MSDs found during the single-nanoparticle tracking (Chap. 6.2). Instead, figure 6.11, A depicts 20 arbitrarily chosen representants from the whole set MSDs obtained from measurements of one particular embryo. By looking at the arrays of MSDs (Fig. 6.11, A and B) and the averaged MSDs (Fig. 6.11, C and D) it turned out that the embryo types (His-EGFP and His-EGFP/kin-5-Halo) do not appear particularily different. A deeper analysis of the MSDs in terms of anomaly parameters will clearify to what extend dynamics of CNTs in His-EGFP and in His-EGFP/kin-5-Halo embryos differ.

\subsubsection{Anomaly parameters}

To assess the diffusive behavior of CNTs in Drosophila embryos, we make use of the so called anomaly paramter $\alpha$. The anomaly parameter is the exponent describing the power law in (Eq. 6.8) and is commonly used to numerically distinguish sub-diffusive, diffusive and super-diffusive motions [167]. Molecular motors of the kinesin family usually display directed, i. e. super-diffusive motility [6]. We have analyzed $\alpha$ by fitting the anomaly parameter to two different lagtime regimes $\alpha_{1}(\tau \leqslant 1 \mathrm{~s})$ and $\alpha_{2}(\tau \leqslant 5 \mathrm{~s})$ of different interval lengths (Fig. 6.11, C). The corresponding histograms in (Fig. 6.13) of $\alpha$ both reflect the behavior seen in (Fig. 6.10) in the two embryo types. When comparing upper and lower panels of (Fig. 6.13, A and B), we observe that for both embryo types (His-EGFP and His-EGFP/kin-5-Halo, yield of linear fits for the $\alpha$ decreases with trajectory lifetime. This is because the tracking revealed short trajection times. We speculate that CNTS are pulled out of the focal plane while the nuclear array starts to reorganize in phases of enhanced cytoplasmic flow. In the course of our studies it turned out, that $\alpha_{1}$ and $\alpha_{2}$ showed outliers. Data sets in 
both embryo types (His-EGFP and His-EGFP/kin-5-Halo even contained negative values for $\alpha_{1}$ and $\alpha_{2}$. This often led to datasets with $\alpha \gg 1$ or even negative medians of $\alpha_{1,2}$. Hence, it is reasonable to find a way to detect pathological fits by looking at the errors and exclude fits which are obviously not reflecting the physical dynamics of CNTs in the embryos. For that purpose, we have compared four commonly used error models (mean absolute error (MAE), root-mean-square error (RMSE), normalized root-mean-square error (nRMSE) and coefficient of variation of the root-mean-square error $(\mathrm{CV}(\mathrm{RMSE}) \mathrm{f})$ to yield fits for $\alpha$, which are physically reasonable:

$$
\begin{aligned}
\text { MAE } & :=\frac{1}{N} \sum_{k=1}^{N}\left|\left\langle x^{2}\right\rangle_{k}-{\widehat{\left\langle x^{2}\right\rangle_{k}}}\right| \\
\text { RMSE } & :=\sqrt{\frac{1}{N} \sum_{k=1}^{N}\left(\left\langle x^{2}\right\rangle_{k}-\widehat{\left\langle x^{2}\right\rangle_{k}}\right)^{2}} \\
\mathrm{nRMSE} & :=\sqrt{\frac{1}{N} \sum_{k=1}^{N}\left(\frac{\left\langle x^{2}\right\rangle_{k}-\widehat{\left\langle x^{2}\right\rangle_{k}}}{\widehat{\left\langle x^{2}\right\rangle_{k}}}\right)^{2}} \\
\mathrm{CV}(\mathrm{RMSE}) & :=\operatorname{RMSE} /\left(\frac{1}{N} \sum_{k=1}^{N}\left\langle x^{2}\right\rangle_{k}\right) .
\end{aligned}
$$

In definitions (Eq. 6.9 6.12) measured values appear undecorated, while fit values carry hats. Comparing these errors (Fig. 6.14) is important in order to choose an appropriate model to use in further studies, and by applying them to our fits discard unreasonable fits of $\alpha$, which have too large residuals. For that purpose, we studied the histograms of these errors (Fig. 6.14) and chose appropriate error models according to quantile analysis of pooled anomaly parameters from pooled data of His-EGFP and His-EGFP/kin-5-Halo embryos (Tab. 6.3). We chose the least tolerant error models according to quantile analysis (Tab. 6.3), which turned out to be the MAEs and RMSEs. In order to exclude as many failed fits on noisy data as possible, we combined MAEs and RMSE; for the given set of MSDs to have a sharp and strict criterion to assess the suitability of the fits. From these error-corrected fits of MSDs we computed the distribution of $\alpha$ over trajectory lifetime (Fig. 6.15). From the analysis of the anomaly parameters it can be seen (Fig. 6.12) that both embryo groups (His-EGFP and His-EGFP/kin-5-Halo) exhibit predominantly diffusive or sub-diffusive behaviour with fits at longer intervals $\left(\alpha_{2}\right)$ tending to show higher values for the anomaly parameters. As can be seen in (Fig. 6.15), medians of $\alpha_{1}^{*}$ and $\alpha_{2}^{*}$ in these two groups barely differ. In the present case, His-EGFP/kin-5-Halo embryos showed track lifetimes that were up to one order of magnitude longer than those of His-EGFP embryos. However, due to the large variety in total lifetime of CNT trajectories in embryos the trajectory lifetime would not be an ideal criterion for making distinctions between these groups. It is also possible, that on long track lifetimes particular CNTs show up as not moving. In our experiments it turned out, that the total number of trajectories varied within each individual embryo, 
independent of the transgenic cross. Concerning the distinction criterions based on MSD analysis a more in depth study is recommended. 

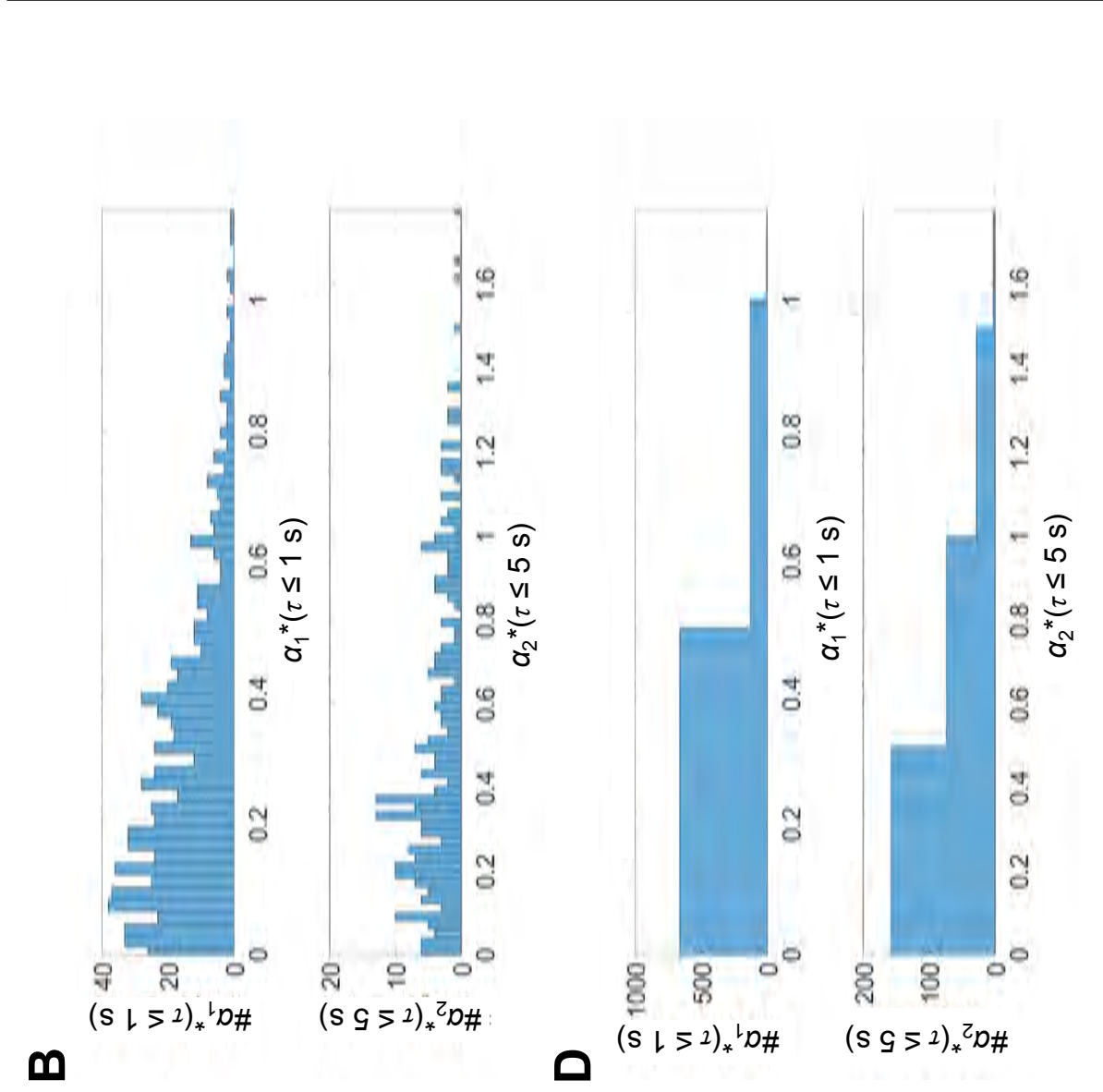

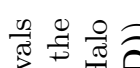

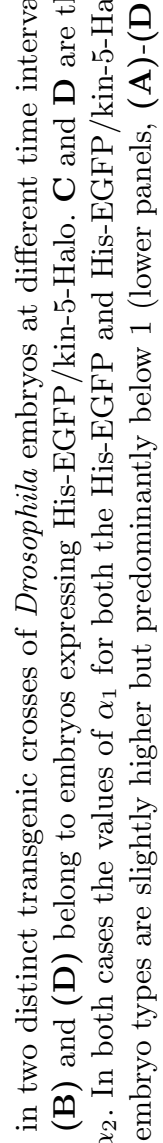

$\mathbf{m}$

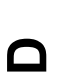

$\left(s\llcorner>2)_{*}{ }^{l} D \# \quad(s \in>1)_{*}{ }^{2} D \#\right.$
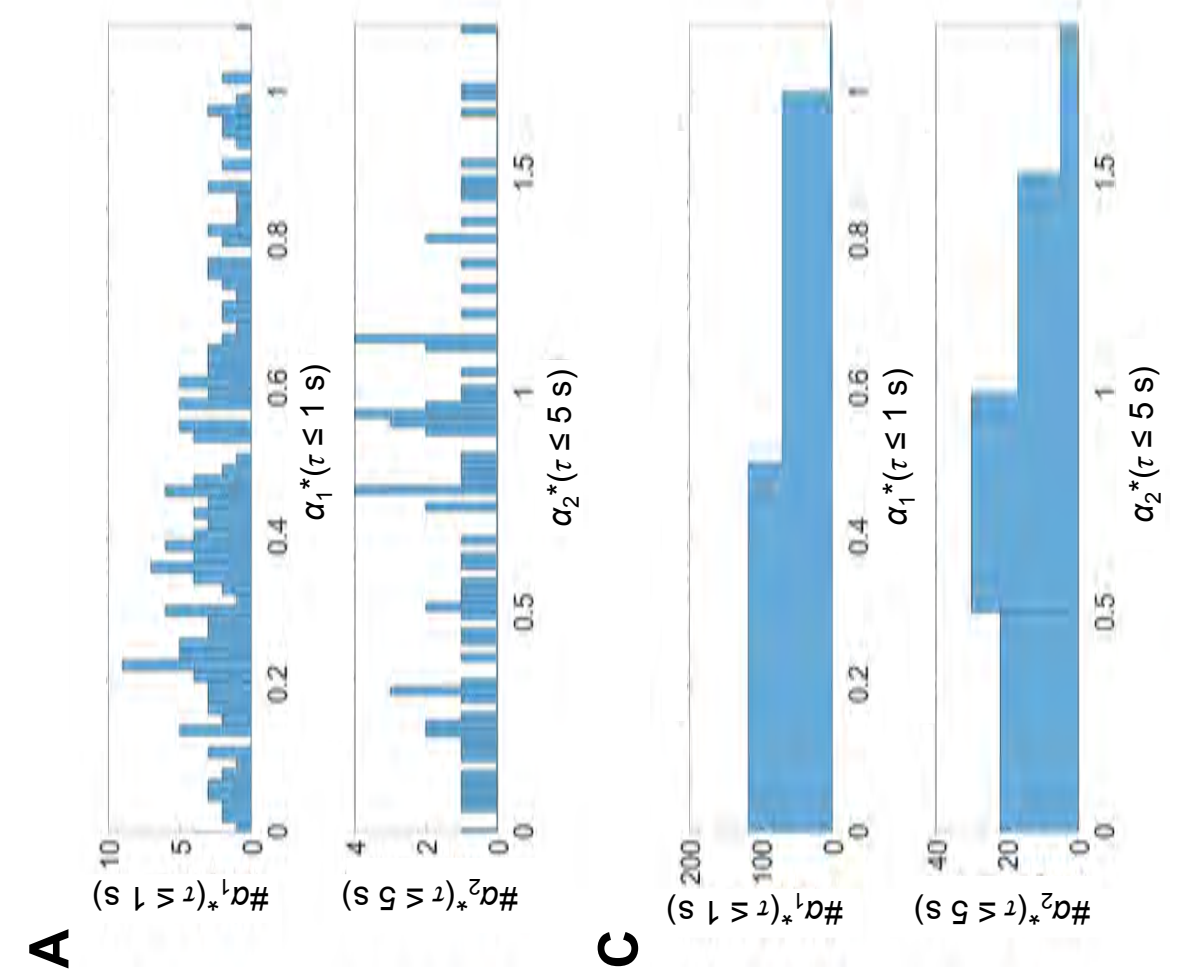

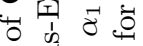

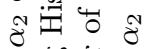

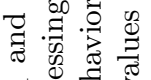

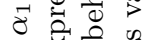
क्षे

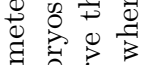
芯 द्वे สํ.

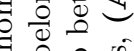
สี $\stackrel{0}{\circ} \frac{0}{8}$

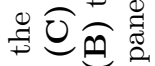
苟 चี

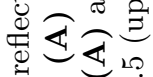

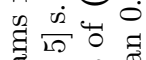

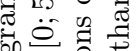

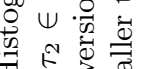

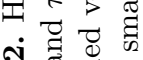
늘 i。

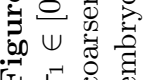




\section{A}
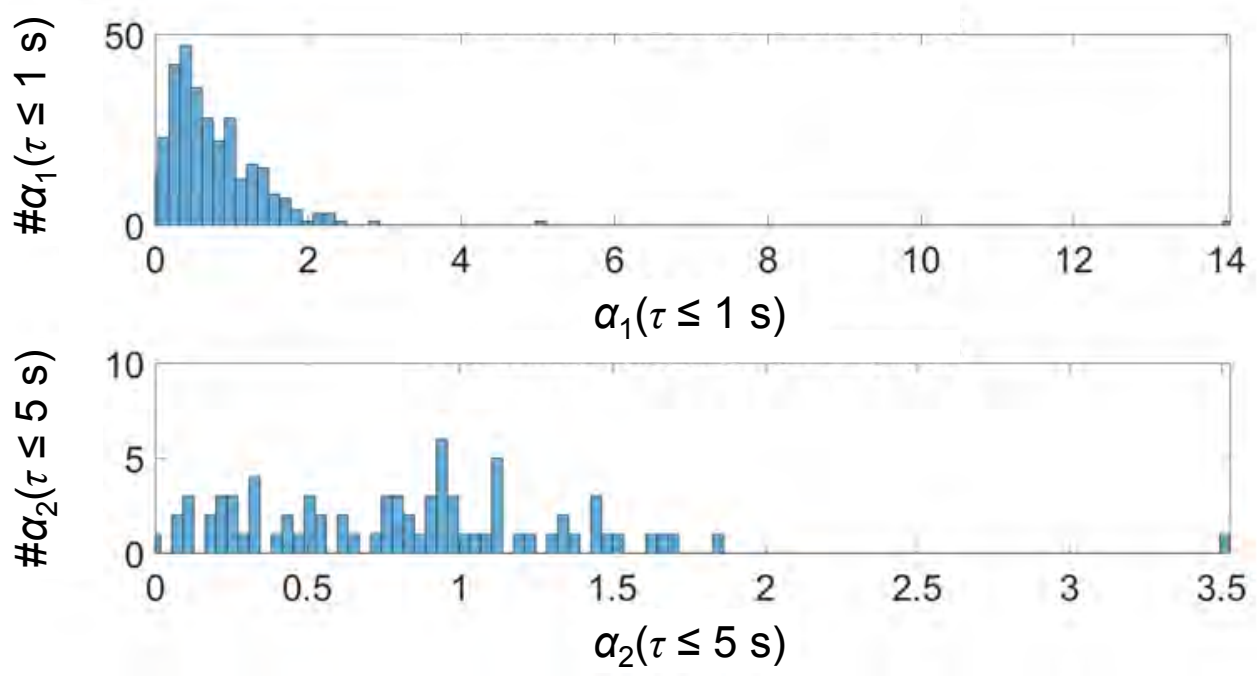

B
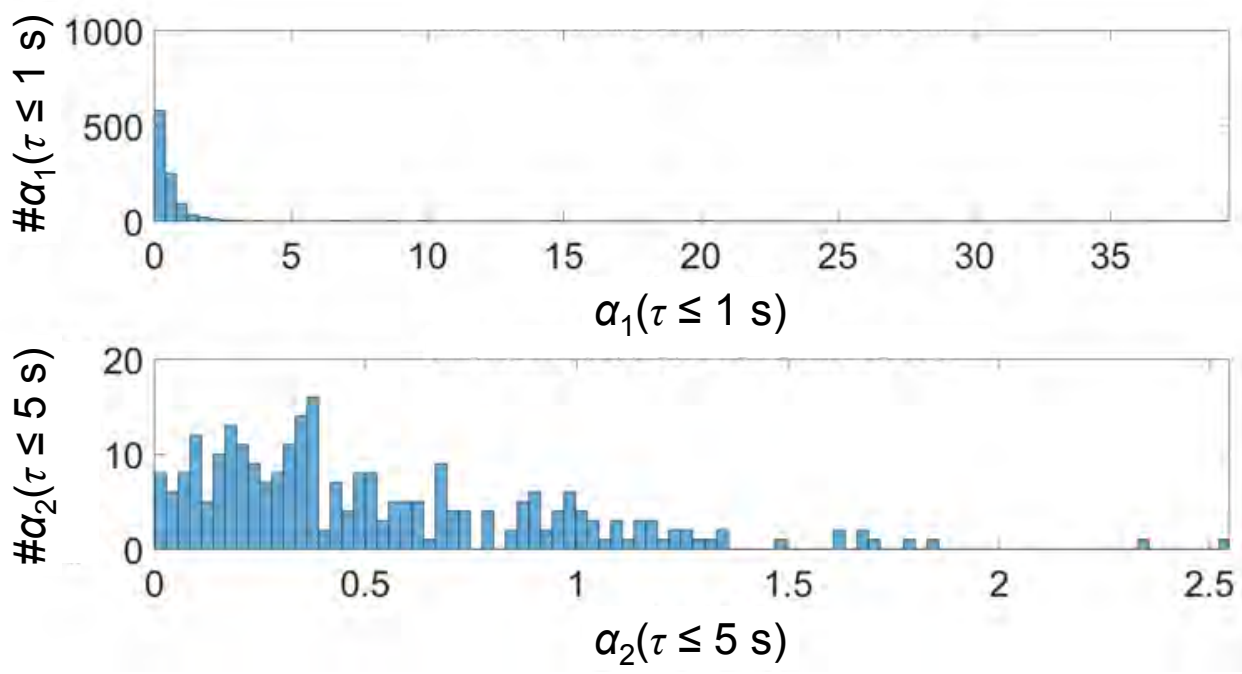

Figure 6.13. Statistics of the anomaly parameters $\alpha_{1,2}$ for short and long track lifetimes. The upper panels in (A) and (B) show the histograms of the anomaly parameter $\alpha_{1}$ of individual CNTs in an embryo expressing His-EGFP (A) and an embryo expressing His-EGFP/kin-5-Halo (B) during a short interval $\tau_{1} \in[0 ; 1] \mathrm{s}$ for the linear fit of the measured MSD The lower panel shows the histograms of the anomaly parameter $\alpha_{2}$ of individual CNTs in an embryo expressing His-EGFP (A) and an embryo expressing His-EGFP/kin-5-Halo (B) during a long time interval $\tau_{2} \in[0 ; 5] \mathrm{s}$ for the linear fit of the measured MSD.

Table 6.3. Quantiles of four error models. Table contains values of pooled data from His-EGFP embryos and His-EGFP/kin-5-Halo embryos. The first two columns define error-corrected anomaly parameter $\alpha_{1,2}^{*}$.

\section{Quantile MAE RMSE nRMSE CV(RMSE)}

$\begin{array}{lllll}75 \% & 0.19 & 0.21 & 0.91 & 5.72 \\ 95 \% & 6.78 & 8.47 & 0.99 & 50.20\end{array}$




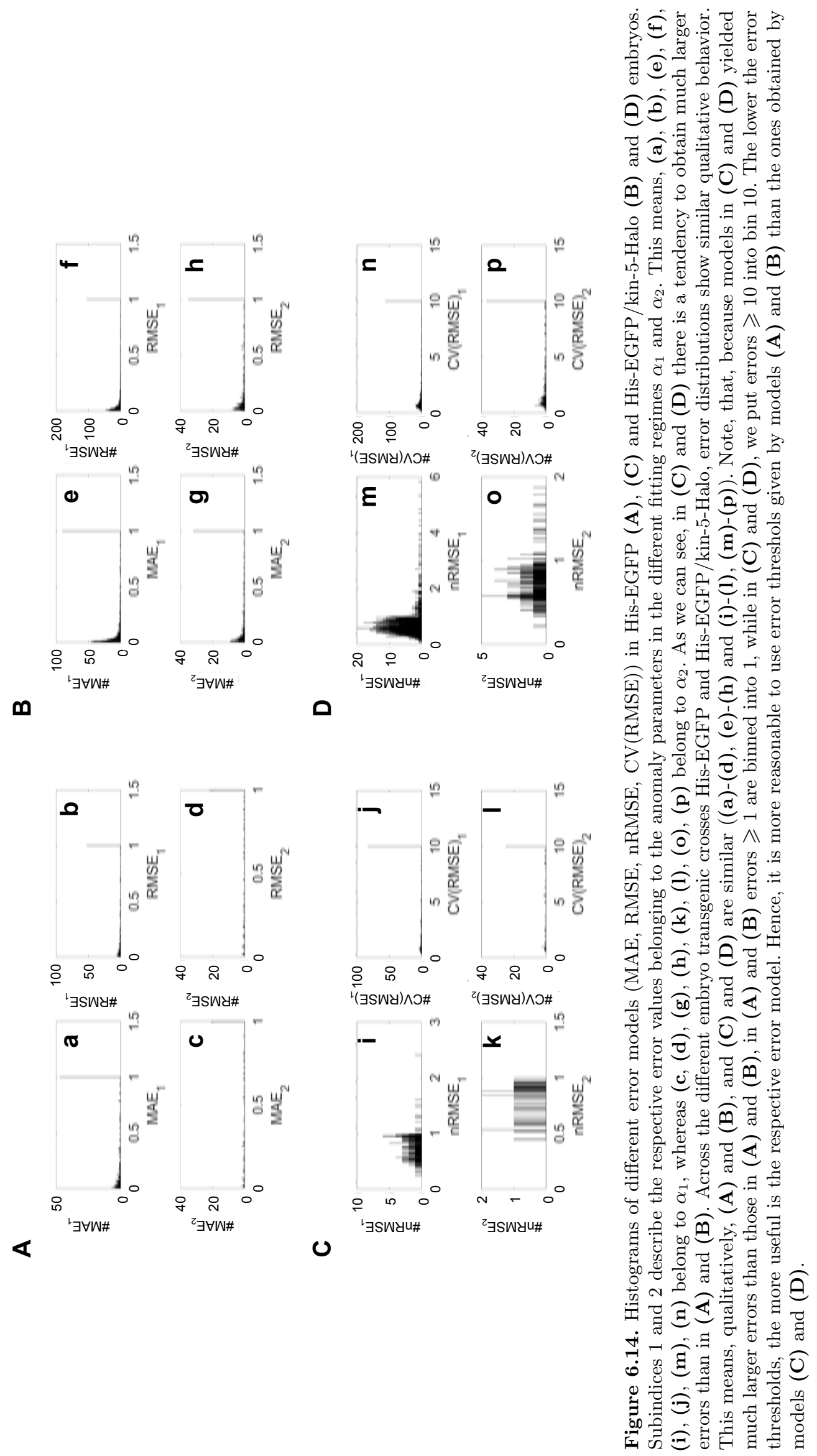



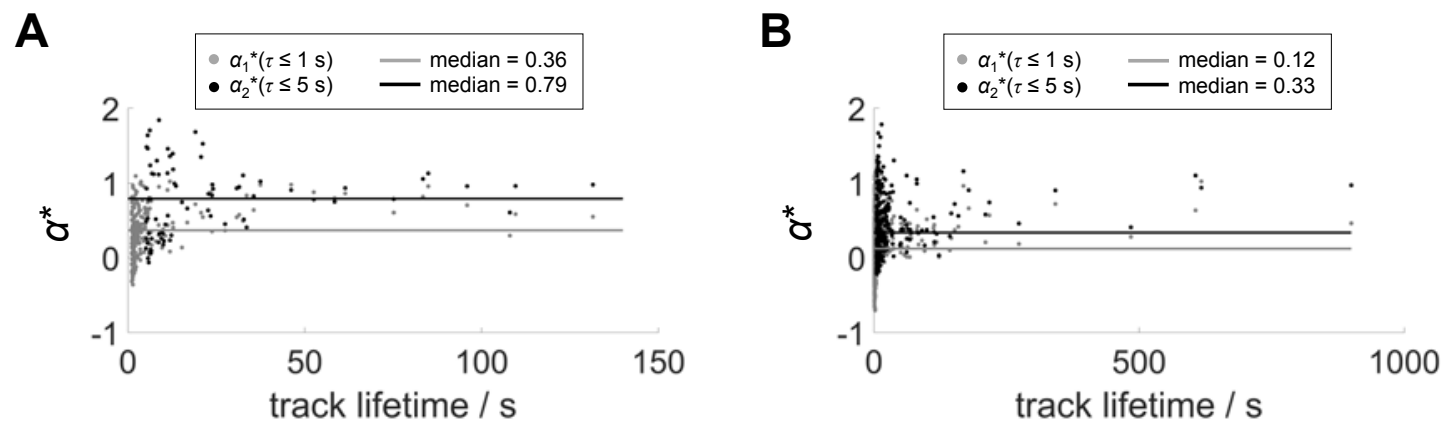

Figure 6.15. Scatter plot of the values of the MAE RMSE corrected anomaly parameters $\alpha^{*}$ vs. trajectory lifetimes. A. Values of the MAE $\cap$ RMSE corrected anomaly parameter $\alpha^{*}$ of the linear fit of MSD in an embryo expressing His-EGFP B. Values of the MAE RMSE corrected anomaly parameter $\alpha^{*}$ of the linear fit of MSD in an embryo expressing His-EGFP/kin-5-Halo

\subsubsection{Diffusivity}

After having analyzed anomaly parameters in His-EGFP and His-EGFP/kin-5-Halo embryos, we now look at the diffusivity $D$ of CNTs in embryos of both transgenic crosses. Diffusivity links the MSD physically to viscous material properties. Microscopic viscosity in syncytial Drosophila embryos has already been investigated using mesoscopic beads [5]. Here we want to set the fundament for further studies by using non-bleaching and non-blinking single-nanoparticles to establish CNTs as microscopic probes for micromechanical investigations. Diffusivity and MSD are connected via (Eq. 6.8). From the tracks of individual CNTs, we have determined the diffusivity $D_{\alpha}$ resulting in the histogram shown in figure 6.16. From (Fig. 6.16), we learn that diffusivity in His-EGFP and His-EGFP/kin-5-Halo embryos both show no significant difference.

A

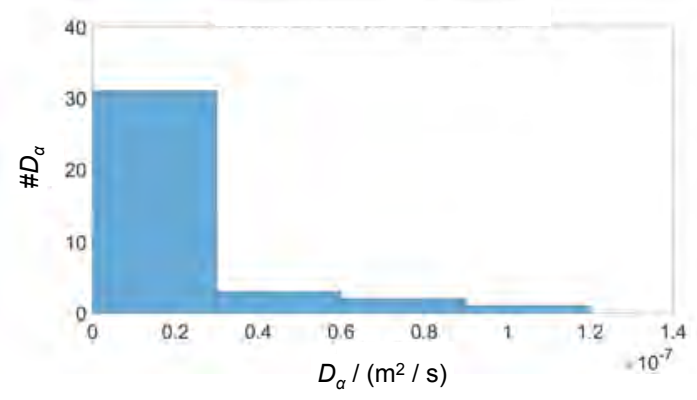

B

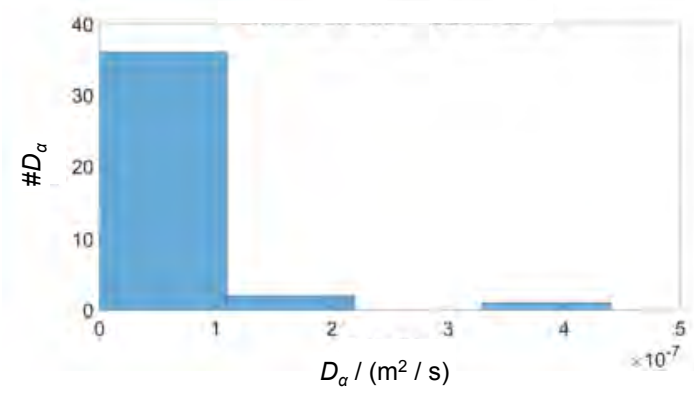

Figure 6.16. 16 Distribution of diffusivity $D_{\alpha}$. A. Diffusivity $D_{\alpha}$ for an embryo expressing His-EGFP B. Diffusivity $D_{\alpha}$ for an embryo expressing His-EGFP/kin-5-Halo

\subsubsection{Velocity cross-correlations of individual carbon nanotube trajectories in living Drosophila embryos}

In the course of our single-nanoparticle studies, we so far have considered the behavior of individual CNTs by looking at spatial autocorrelations (MSDs). The basis for the 
MSD studies are the tracking studies (Chap. 6.2). MSD analysis provides an adequate tool to obtain information about the behavior of individual particles embededded in a viscous environment. It gives valuable information about the diffusive behavior of CNTs. However, it does not provide information about how the individual parts of the tissue interact with each other. A complete picture of the rich local interactions inside developing embryos needs investigation of cross-correlations of the CNTs found by tracking (Chap. 6.2). Furthermore, these cross-correlations have the potential to be used as dynamic order parameter for characterization of the correlative motion of single CNT molecules in different types of Drosophila embryos. Here, we here consider the cross-correlation of velocities of individual CNTs [1]. We have computed velocity correlations of CNTs in Drosophila embryos expressing His-EGFP and His-EGFP/kin-5-Halo to compare the correlative behavior of CNTs in these two transgenic crosses. From analysis of distance-dependent velocity correlations of pairs of CNTs, we obtain a distant-dependent dynamic order parameter for comparison of the dynamical behavior of CNTs in the two transgenic crosses.

We define the distance-dependent two-particle velocity cross-correlation $C(R)$ by [1]:

$$
C\left(R_{k}\right):=\left\langle\mathbf{v}_{\alpha}\left(\overline{\mathbf{r}_{\alpha}}, t_{k}\right) \bullet \mathbf{v}_{\beta}\left(\overline{\mathbf{r}_{\beta}}, t_{k}\right) \delta\left(\left|\overline{\mathbf{r}_{\alpha}\left(t_{k}\right)}-\overline{\mathbf{r}_{\beta}\left(t_{k}\right)}\right|-R_{k}\right)\right\rangle
$$

(Eq. 6.13) shows the scalar products of two particles appearing at the same time, i.e. the same frame, whereupon these scalar products are sorted according to the distance between these particles as indicated by the $\delta$ function. To bin the scalar products according to their distance $R$, we use a reasonable tolerance window $R=R^{\prime}+\delta R$. We illustrate this with a hypothetical example:

$$
C(R=50 \mu \mathrm{m})=\frac{\left(\mathbf{v}_{14} \bullet \mathbf{v}_{15}\right)_{t_{25}}+\left(\mathbf{v}_{3} \bullet \mathbf{v}_{92}\right)_{t_{49}}}{2} .
$$

In (Eq. 6.14), a hypothetical velocity cross-correlation of four particles (i. e. in our experiments this would be CNTs 3, 14, 15, 92 is calculated. In this example, particles 14 and 15 appear in frame 25 and particle pair 3 and 92 appear in frame 49. After computing the distances within all particle pairs per frame within the range of the respective frame set, it so may turn out that the pairs 14, 15 and 3, 92 have a distance of $R=50 \mu \mathrm{m}$ to each other. The denominator in (Eq. 6.14) counts the number of summands in the numerator. Sorting the values of $C$ according to their distances $R$, provides histograms (Fig. 6.17, A, C). To evaluate distance-dependent two-particle velocity correlations (Eq. 6.13), we use the data obtained in (Chap. 6.2). With selfwritten MATLAB software, we computed pairwise cross-correlations of individual CNT trajectories defined by (Eq. 6.13). After extracting the raw trajectories of individual CNTs provided by TrackNTrace, implementation of (Eq. 6.13) is straightforward. Numerically, velocities are approximated by the average of positions amidst the trajectory points. Thus, in order to define velocities, we first have to define the locations of particular velocities. For that, we shift the origin of a point $\mathbf{r}=r_{j} \mathbf{e}_{j}$ on a trajectory at time point $t_{k}$ about half midway to the next trajectory point at time point $t_{N}$. Thus, we can assign an exactly defined position to each velocity vector. In order to define the projection of two adjacent velocity vectors on one another (i. e. $\mathbf{v}_{\alpha} \cdot \mathbf{v}_{\beta}$ ), we first have to define their positions by averaging distances: 


$$
\overline{r_{j, \zeta}\left(t_{k+N}\right)}:=r_{j, \zeta}\left(t_{k}\right)+\frac{r_{j, \zeta}\left(t_{N}\right)-r_{j, \zeta}\left(t_{k}\right)}{2} .
$$

We then approximated velocity field vector components $v_{j}$ by the average speed of particles incated by $\zeta=\{\alpha, \beta\}$ :

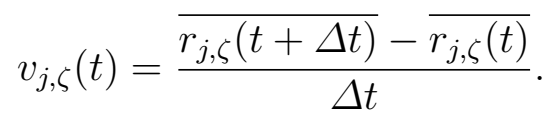

We then compute distances of particle pairs $(\alpha, \beta)$ in each frame and bin them according to their distance $R_{k}$ at timepoint $t_{k}$ which we then bin according to their distances (Fig. 6.17).
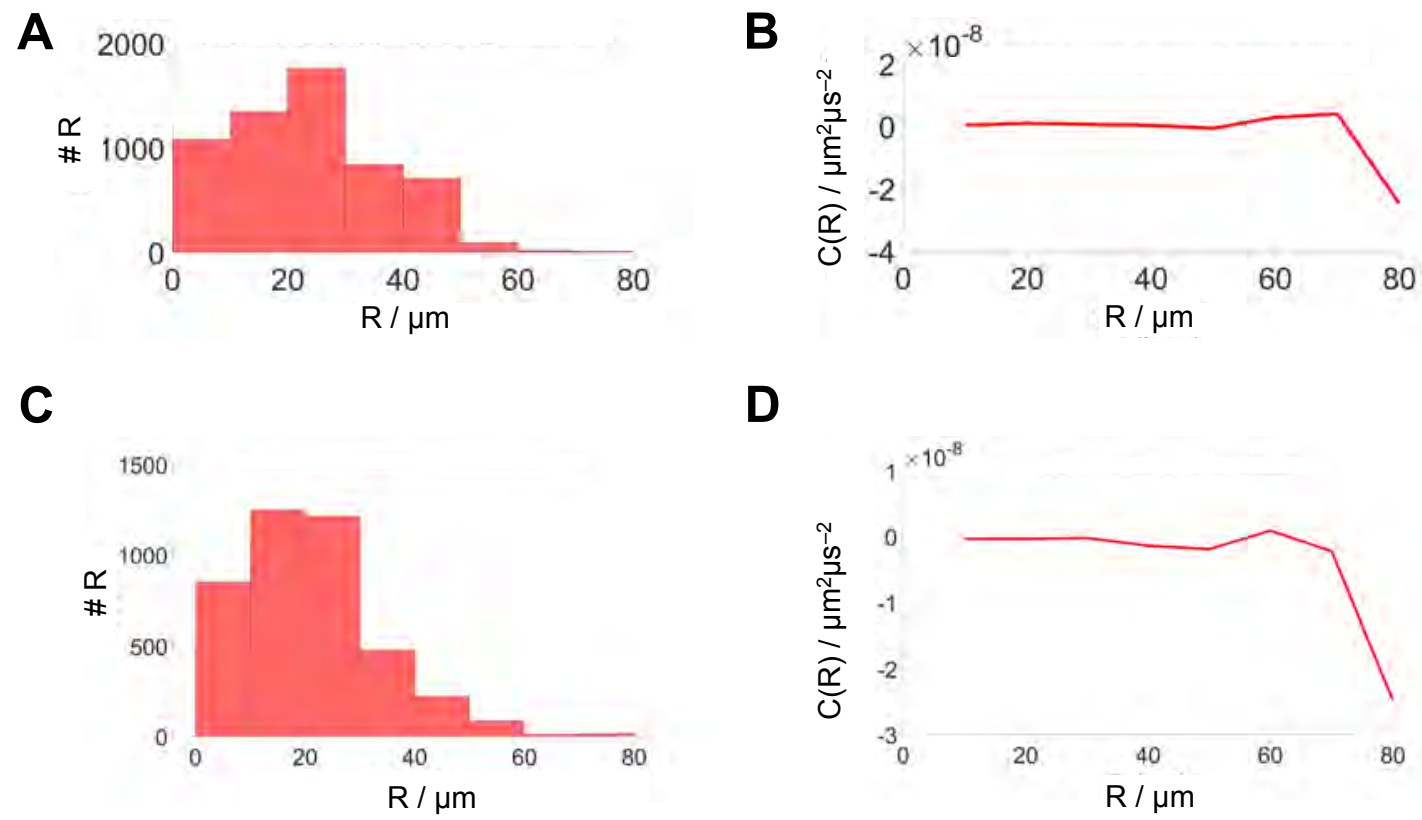

Figure 6.17. Velocity correlations in Drosophila embryos. (A) and (C) show histograms of distances $R$. (B) and (D) show correlations between two CNTs which were found during overall recording time. Most velocity correlations of the CNTs are found at short and intermediate distances. CNTs are mostly uncorrelated fluctuating around zero. For long distances, a decrease in $C(R)$ for long times is observed, indicating anti-correlative behavior of CNTs The two types His-EGFP A, B and His-EGFP/kin-5-Halo A, B) show similar behavior.

As the distance-dependent velocity cross-correlations over distances $R$ of individual CNT tracks per image reveal, cross-correlations of CNTs in both embryos exhibited the same tendency of decreasing $C$.

\subsubsection{Hydrodynamic length of carbon nanotubes in living Drosophila embryos}

Transport behavior of tracer particles such as CNTs in viscous media such as living Drosophila embryos is strongly influenced by their friction and thus by their geometry [169, 170]. The hydrodynamic length $L$ provides a measure for relating geometry of the tracer particle and its diffusive behavior in a medium with viscosity $\eta$. We define 
$L$ as the length of a hypothetical rod-shaped particle displaying the same diffusive behavior in a solvent with viscosity $\eta$ as a CNT would do [171]. Hydrodynamic lengths of CNTs have already been studied in in vitro sedimentation experiments [172. In Drosophila embryos experiments with mesoscopic beads yielded results for the microscopic viscosity $\eta[5]$. Here we have measured the hydrodynamic length of CNTs in living Drosophila embryos by analyizing MSDs obtained from NIR fluorescence imaging of CNTs. MSD $\left\langle x^{2}\right\rangle$ of a particle and its diffusivity $D_{\alpha}$ in our 2D system are linked via (Eq. 6.8), yielding a power law for lag time $\tau$. Since our data showed predominantly diffusive or sub-diffusive behavior of CNTs in Drosophila embryos we consider the subset of our measured data with anomaly parameters in the range of $\alpha=1 \pm 0.1$. From the $\left\langle x^{2}\right\rangle$ of CNTs in Drosophila embryos we obtain $D$ directly from the offset in the logarithmic fit of $\left\langle x^{2}\right\rangle$. We now connect geometry and diffusivity via friction $\xi$ by [170]

$$
D_{\alpha}=\frac{k_{\mathrm{B}} T}{\xi} .
$$

Hence, we can compute hydrodynamic length $L$ of a average single CNT by using the solution of the diffusion equation for a rod-shaped particle [173]

$$
L=\frac{k_{B} T \ln \left(\frac{L}{d}\right)}{3 \pi \eta D_{\alpha}}
$$

with friction $\xi=\ln (L / d) /(3 \pi \eta L)$ [173], tube length $L=1 \mu \mathrm{m}$ and tube diameter $d=1 \mathrm{~nm}[6,71$. Here, we use a microscopic viscosity in the Drosophila embryo of $\eta=4 \mathrm{mPa} \cdot \mathrm{s}[5]$ at standard conditions. From this, we obtain an effective length $L$ for CNTs in Drosophila embryos of

$$
L=(71 \pm 10) \mathrm{nm} .
$$

This result can be used as a tool for further research on the transport behavior of CNTs in Drosophila embryos, for instance when using CNTs as sensors in biomedical applications.

\subsection{Mesoscopic bulk dynamics: Particle image velocimetry with fluorescent carbon nanotubes}

After having observed and systematically characterized behavior of single CNT molecules during "silent" phases, i. e. phases with suppressed cytoplasmic flow, we now want to demonstrate that combining CNT; and PIV] can be used to probe bulk dynamics during phases exhibiting enhanced cytoplasmic flow. Little is known about the contributions of the cytoplasm during the reordering of the nuclear array, which is important for embryonic development [64]. Due to the embedding of the nuclear array in the cytoplasm, influence of cytoplasmic flow on the morphological upheavals in the cortex seems reasonable. However, not much is known about the biological function and driving physical mechanisms of cytoplasmic flow 63.

In the following, we present a method of combining excellent photophysical properties of CNTs with the non-invasive particle tracking with $\mathrm{PIV}$ and thus open the door 
for new efficient methods for the quest for the underlying physical mechanisms of cytoplasmic flow. In this chapter, we give a proof-of-concept, that it is possible to detect mesoscopic bulk dynamics in living Drosophila embryos using CNTs as photostable NIR fluorescent molecular probes in combination with PIV. PIV is a noninvasive method to observe and quantify velocity fields and thus to characterize flow behavior [174]. Unlike single-particle tracking (Chap. 6.2), where individual particles are detected locally, particle detction with $\mathrm{PIV}$ is based on averaging movements within previously defined spatial regions. For averaging, the entire image is covered by a grid determining the final resolution of the cells within which averaging will take place by comparison with surrounding frames. We injected CNTs into embryos expressing His-EGFP and, by this, prove that CNTs can also be used for mesoscopic studies of the cytoplasm. We show that CNTs can be applied as fluorescent probes for quantitative imaging of velocity gradients of cytoplasmic flow and show in further processing, that CNTs are appropriate to be used in PIV.

In the single-nanoparticle experiments in this work, it turned out, that particular CNTs are only trackable in the range of few seconds, since they move out of focus, making it single-nanoparticle tracking in Drosophila difficult. Thus, by applying PIV, one can observe further interesting features during cortical upheavals which remain covered in single-nanoparticle imaging of CNTs. Other than in single-nanoparticle experiments, by studying dynamics with PIV, we are not constrained to the dispersion state during imaging individual CNTs. This facilitates to a large extend continuous observation of intracellular dynamics on long time scales. Data acquisition protocols used for PIV studies are basically the same as those used for single-nanoparticle experiments (Chap. 6.1.1) with the only difference that closing intervals of mechanical shutters are shortened to $t_{\text {shut }}=5 \mathrm{~s}$. For this purpose, we have recorded His-EGFP embryos in their interphase. We exposed the whole embryo for $30 \mathrm{~min}$ to $770 \mathrm{~nm}$ laser light of the Ti:Sapph laser to capture cytoplasmic flow at least one interphase. The $488 \mathrm{~nm}$ laser excites His-EGFP each $5 \mathrm{~s}$ (Chap. 6.1.1). In this course, the nuclei drift about $10 \mu \mathrm{m}$, which is about two nuclear diameters. In order to reduce noise, images were lowpass filtered by averaging over 10 consecutive frames. The average longpass filtered image $I_{\text {avg LP, }, k}$ was constructed by computing the arithmetic mean of ten images:

$$
I_{\text {avg LP }, k}=\frac{1}{10} \sum_{j=1}^{10}\left(I_{j}\right) \text {. }
$$

We then used the set of $\left\{I_{\text {avg LP, }, k}\right\}$ as input for the actual PIV analysis using the open-source software PIVlab [175 177]. For further processing, we projected the data obtained by PIVlab $(320 \times 256)$ on a coarse-grained grid measuring $15 \times 19$ with each element having $5 \times 6$ pixels. In order to suppress noisy regions with low particle density, we filter out velocities which were above a given threshold.

To describe the degree of order during reorganization in Drosophila embryos, it seems natural to define a dynamic order parameter. In the following we demonstrate, that combining [CNT imaging in the cytoplasm and PIV] allows the extraction of dynamic order parameters in phases of enhanced cytoplasmic flow by giving a simple example for a possible angular order parameter. 
A

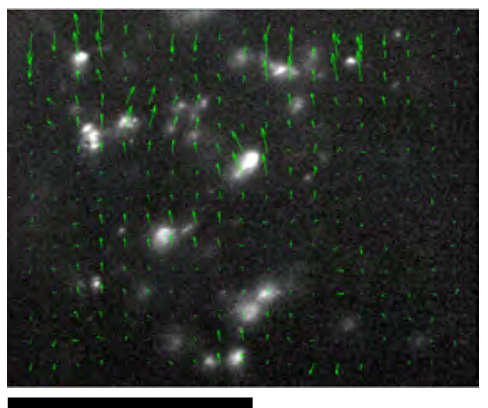

$50 \mu \mathrm{m}$
B

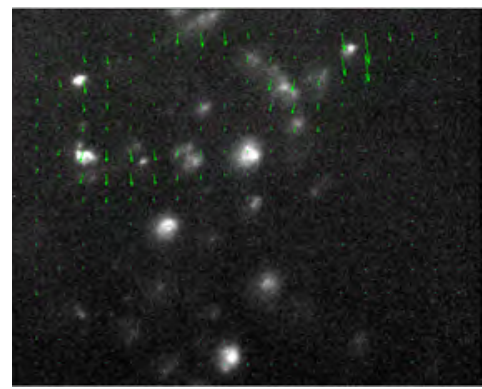

D

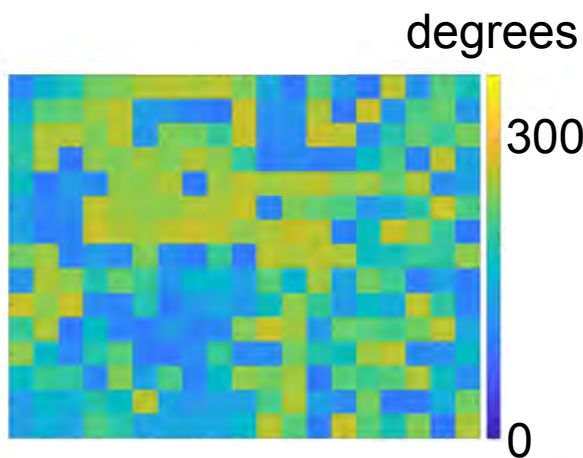

Figure 6.18. Cytoplasmic flow in Drosophila embryos obtained from combining CNTs imaging and PIV Flow field vectors of CNTs in Drosophila embryos (A) and (B) with corresponding phases $\phi$ (C) and (D).

From the velocity vector fields obtained by the PIV] analysis, we extracted particular orientation of velocity field representant $\mathbf{v}$ with respect to one axis and the absolute value $\mathbf{v}:=\left(v_{x}, v_{y}\right)$. Together with the absolute value $v:=\sqrt{v_{x}^{2}+v_{y}^{2}}$ of the velocity field representants, we can define an angle

$$
\phi:=\arctan \left(\frac{v_{y}}{v_{x}}\right) .
$$

between $x$-axis and velocity representant $\mathbf{v}$ detechted by [PIV] which can be regarded as an example for a dynamic order parameter obtained from combining CNT imaging and PIV]

In further research on intracellular reorganization processes in embryonic Drosophila. generalization of $\phi$ can be established by correlating values of $\phi$. In this thesis, however, we limit ourselves to demonstrating the successful applicability of the PIV method to fluorescence signals of CNTs in living syncytial Drosophila embryos by referring to this example.

In conclusion, this chapter, we delivered a proof-of-concept, that $\mathrm{PIV}$ can be used to characterize mesoscopic dynamics of the cytoplasmic flow inside syncytial Drosophila embryos using NIR fluorescent CNTs as tracer particles of molecular size. 



\section{Discussion}

\subsection{Carbon nanotube preparation and microinjection}

The individual steps during CNT 3 uspension required a high degree of trained and skilled craftsmanship in order to produce reproducible results. The dissolution of ssDNA into water was essentially uncomplicated. In contrast, the handling of CNTs in all further steps of the CNT sample preparation turned out to be quite challenging. Firstly, this concerned the portioning of dry CNTs for weighing, since CNTs have a low mass density and a high sensitivity to electrostatic interactions. This means that despite grounding, CNTs adhered to all surfaces which they came into contact with. The CNT masses were difficult to measure due to the measuring tolerance of the scale (CP224S; Sartorius AG, Göttingen, Germany). As a possible solution to both problems, we propose to use specified and precisely weighed commercial CNT batches in future studies.

During the subsequent sonication of the CNT suspension a considerable amount of CNTs remained visibly attached to the sonication probe. Also after sonication macroscopically visible remains of wet CNTs were found attached to the inner walls of the glass scintillation vial used for sonication. These residues became visible when the CNT suspensions were transferred to an Eppendorf tube for centrifugation. As a result, an unknown amount of CNTs was lost before centrifugation. To fully disperse CNT aggregates between CNTs we sonicated the sample with $10 \mathrm{~W}$ over 90 min, causing to break up inter-tube van der Waals bonds. Determination of the effects of the sonication parameters used on SsDNA-wrapping was beyond our control. Further investigations are recommended to clarify how much the dispersion state of suspensions is affected by possible SsDNA damage.

Overall a high degree of practice and experience with the individual steps allowed us to create clearly visible CNTs during NIR imaging of individual CNTs in living Drosophila embryos.

As well as the suspension of the CNTs, the functionalization of individual CNTs with a SSE HaloTag ${ }^{\circledR}$ ligand proved to be demanding. A major concern with functionalization with SSE HaloTag ${ }^{\circledR}$ ) ligand is the ambiguous detectability of the successful covalent bond between the the SSE and the AminoC12 $5^{\prime}$ end of the ssDNA in vitro. The HaloTag ${ }^{\circledR}$ method provides non-invasive tagging of single kinesin-1 motor proteins in living cells [6].

In this thesis we have applied this method to kinesin-5 motor proteins in living 
transgenic Drosophila embryos. However, as it turned out, the tracks of individual CNTs in His-EGFP and His-EGFP/kin-5-Halo embryos showed similar behavior. Furthermore, during imaging, individual CNTs were only visible for less than a minute. A successful tagging of HaloTag ${ }^{\mathbb{R}}$.modified kinesin-5 also required that the His-EGFP/kin-5-Halo embryos all expressed HaloTag ${ }^{\circledR}$. modified kinesin-5 motor proteins. This poses the question, if in fact a successful covalent bond of functionalized SsDNA-wrapped CNTs inside the His-EGFP/kin-5-Halo embryos was created.

Still, our studies provided a useful demonstration of the capabilities of our setup for long-time imaging of NIR CNTs combined with VIS imaging in Drosophila.

All in all, we note that the process based on the one in [6] for the production of functionalized CNTs for our special application in Drosophila embryos despite the many, partly great challenges, yielded successful single-nanoparticle imaging including single-nanoparticle tracking and optical characterization of individual CNT molecules in tissue of living Drosophila embryos.

To introduce CNTs suspensions into Drosophila embryos we used microinjection. In this work we learned that the needle position during microinjection of CNT suspensions into Drosophila embryos influences the distribution of CNTs in the embryo. This is why we lined up the embryos in the middle of the coverslip for minimal position corrections during imaging. We recognized that an injection along the short embryo axis usually resulted in a higher yield of homogeneously dispersed CNTs throughout the embryo.

The injection needle was a custom-made tapered glass capillary of which the closed tip had to be removed by carefully clipping it off. This resulted in different diameters of the needle outlet which we estimated to about $50 \mu \mathrm{m}$. Those different diameters and tissue clogging the needle outlet made each injection different from one another. However, an experienced handling of the needle led to the observation of individual CNT trajectories in Drosophila mbryos.

\subsection{Imaging}

In this work, we have used an inverted microscope enabling simultaneous imaging of NIR CNTs and VIS His-EGFP. We used a mechanically stabilized sample stage and equipped the bottom side with two pairs of rare-earth magnets. These magnets ensured a fixation of the glass coverslip on the sample stage, preventing it from sliding during imaging. We optimized the detector path compared to the structure used in preliminary studies for the detection of CNTs in syncytial Drosophila embryos [133] to allow a higher intensity of CNT fluorescence. Before measuring the NIR fluorescence of CNTs, a two point calibration of the InGaAs CMOS camera was performed. We used a custom-built NIR diode lamp with adjustable intensity to illuminate the detector of the camera completely and homogeneously. It was important to ensure that the InGaAs CMOS sensor received sufficient photons from CNTs using a two point calibration. For this calibration, we recorded a dark image and a bright image. During recording of the bright image, it was important to make sure to not reach upper edge of the dynamic range, as otherwise parts of it would have been cut off and the camera would not provide enough contrast during imaging.

The clearly arranged graphical user interfaces of the selected software enabled 
targeted and uncomplicated control of the respective cameras. Using the open-source control software Micro-Manager, the EMCCD for visible fluorescence detection allowed for reliable control of the mechanical shutter behind the $488 \mathrm{~nm}$ laser. To ensure that we see the same image section in both the NIR and VIS channel, we used fluorescent beads that were visible in both the NIR and VIS channel. We excited the fluorescence of the beads with $561 \mathrm{~nm}$ laser light to calibrate the FOVs. Through this so-called registration process (Chap. 6.1.2) we achieved a clear overlap of both channels. For registration, the smaller picture with the coarser resolution $\left(9.6 \times 7.7 \mathrm{~mm}^{2}, 30 \mu \mathrm{m} /\right.$ pixel, NIR channel, detected with InGaAs CMOS camera) was superpositioned on the larger image with the finer resolution $\left(13.3 \times 13.3 \mathrm{~mm}^{2}\right.$, $13 \mu \mathrm{m} /$ pixel, VIS channel, detected with EMCCD camera). The use of beads turned out to be a fast and efficient way to compare the two FOVs in the channels NIR and VIS, allowing parfocality to be adjusted. Furthermore, the dried beads provided an unmistakable bead pattern in both channels, which could be clearly assigned to the superposition of both FOVs due to the distribution of the dried beads on the coverslip.

We imaged NIR fluorescent CNTs using the high-gain acquisition mode to distinguish the flat CNT fluorescence peaks from the noise floor [152]. In high-gain mode both signal level and noise level are raised compared to low-gain mode. However, high-gain mode offers the decisive advantage that the frame rate can be kept up during imaging and thus lower exposure times can be achieved.

Another challenge during imaging was the behavior of the CNTS in the respective FOV. We found that during imaging, individual CNTs repeatedly moved out of the focal plane, affecting the lifetime of the trajectories. This could be observed especially during the reorganization of the nuclei in the cortex. The two dimensional imaging limited us to tracking CNTs, which in most cases led to a lifetime of detectable CNT trajectories below one minute. In order to ensure persistent tracking of individual CNTS beyond the cortex and to make optimal use of the photostability of the CNTs, we recommend extending the imaging from two to three dimensions for future experiments. For this purpose, a feedback mechanism can be introduced which marks the most intense CNT signals in a reasonable frame interval and tracks them to all three directions using the piezo stage for nanometer precise positioning.

As mentioned above, the dispersion state of the CNT suspension used had large effects on image quality and played a major role in finding a suitable concentration of fluorescent CNTs. Achieving the correct concentration of CNTs also required a lot of experience during injection into the embryos. If there were too few CNTs in the FOV, the sample was not usable due to too few signals. However, a too high concentration of injected CNTs, clear detection became difficult during subsequent tracking. In our case a concentration of 1:50 (CNT suspension : water) proved to be adequate.

In our experiments, anecdotes of directed His-EGFP/kin-5-Halo embryos could be detected, which moved directionally relative to their environment. We were able to extract kymographs (Fig. 6.8) that demonstrated this. However, these velocities were about one magnitued higher higher than expected for kinesin-5 which is normally in the order of 0.02 to $0.10 \mu \mathrm{m} / \mathrm{s}$ [164]. Since directed movements of individual CNTs were observed in both embryo types (His-EGFP and His-EGFP/kin-5-Halo, 
we suspect a nonspecific binding of fluorescent CNTs, for instance to vesicles, which may have conributed to these directed movements.

During the image registration (Chap. 6.1.2) it was noticeable that during the 30minute imaging on average about 17300 instead of the nominal 18000 frames were recorded in the NIR channel (Eq. 6.1). This corresponds to a frame rate of $9.6 \mathrm{~Hz}$ instead of the nominal $10 \mathrm{~Hz}$ (which correspond to $T_{\text {int }}=100 \mathrm{~ms}$ integration time) in the NIR channel. In contrast, the nominal frame rates of 90 frames and 360 frames in $30 \mathrm{~min}$ in the VIS channel, as entered in Micro-Manager (Tab. 6.1), was met. It is likely that the InGaAs CMOS camera uses part of the nominal integration time of $100 \mathrm{~ms}$ to control the internal shutter. We decided to follow the nominal value of the integration time in order to achieve the highest reproducibility possible. In future experiments, this offset can be reduced by optimizing the image registration protocol (Fig. 6.1) by adapting the temporal offset (Eq. 6.4-6.4).

\subsection{Optical properties of carbon nanotubes in living Drosophila embryos}

In this work we have investigated the optical properties of CNTs in living embryos. We found that our CNT samples showed fluorescence maxima at $561 \mathrm{~nm}$ and 770 $\mathrm{nm}$ wavelengths. In order to obtain the results presented in the chapters (Chap. 6.3 we continuously used a Ti:Sapph laser wavelength of $770 \mathrm{~nm}$ to excite CNT NIR fluorescence in syncytial Drosophila Embryos. Here, the adjustable emission wavelength range of the Ti:Sapph laser was a great advantage. Within this range we were able to adjust the wavelength so far that we could get an optimal excitation wavelength in NIR for CNTs with optimal emission peaks. Using the imaging setups presented in Chapter (Chap. 4), we found that the CNTs we used were excitable with a NIR wavelength $(770 \mathrm{~nm})$. We found out that the emission light of the 561 $\mathrm{nm}$ laser provides a lower signal-to-noise ratio than exposure of the same sample with $770 \mathrm{~nm}$. However, it was found that the yield of CNTs in the same [FOV] is higher, which increases the observation of individual events and thus provides a higher, more meaningful data set for statistical evaluation. We have measured the power of the $561 \mathrm{~nm}$ and the Ti:Sapph lasers. These laser powers reached the sample with $287 \mathrm{~mW}(561 \mathrm{~nm})$ and $440 \mathrm{~mW}(770 \mathrm{~nm})$ respectively continuously over a period of $30 \mathrm{~min}$. We found that the structure of the nuclear array inside the embryos remained intact, despite exposure to two lasers (770 nm and $488 \mathrm{~nm}$ ) for excitation of VIS and NIR fluorescence over $30 \mathrm{~min}$. Using a piezo stage for nanometer precise control of individual NIR fluorescent CNTs within living embryos, we successfully measured PSFs to a depth of $45 \mu \mathrm{m}$ within the tissue of living Drosophila embryos. A comparable depth could be achieved by using beads [133]. Thus, we were able to demonstrate that imaging deep in Drosophila tissue is also possible with CNTs. Due to the superior fluorescence properties of CNTs we recommend the use of NIR fluorescent CNTs in further microrheological studies within living Drosophila embryos. 


\subsection{Dynamics of carbon nanotubes in Drosophila embryos}

In this thesis, we have measured the dynamics of living Drosophila embryos in the syncytial stage. A reliable evaluation of the dynamics of single CNTs in singlenanoparticle experiments requires reliable and reproducible tracking. A reliable tracking software is indispensable for a successful further analysis of single trajectories. We consistently used the open-source software TrackNTrace in our single molecule studies. This software was user friendly due to its clear and clean graphical user interface. Compared to other tracking programs like the FIJI plugin TrackMate [178, tracking with TrackNTrace in our special applications turned out to be a more efficient and reliable solution with better performance. The selection of input parameters for the tracking of single nanoparticles presented in this paper, which led to the results presented here, was determined by careful testing of different parameter sets. We were able to find a parameter set that led to a successful detection of single CNTs in living Drosophila embryos. We analyzed the single trajectories with self-written MATLAB software and obtained information about MSDs and velocity cross-correlations (Chap. 6.3). The MSDs could successfully be used as a basis for the calculation of the diffusivity $D_{\alpha}$ and the hydrodynamic length $L$ of functionalized CNTs in living syncytial Drosophila embryos. We also investigated anomaly parameters for two different lagtime intervals $\tau_{1} \in[0 ; 1] \mathrm{s}$ and $\tau_{2} \in[0 ; 5] \mathrm{s}$. With a MAE and RMSE analysis of the plots, the best fits to the MSD measurement data were found. Based on these fitted data we obtained anomaly parameters $\alpha^{*}$, with which we were able to demonstrate diffusive and sub-diffusive behavior of single CNTs in the two embryo types His-EGFP and His-EGFP/kin-5-Halo. From the distributions for $\alpha^{*}$ obtained from CNT trajectories measured in the different embryo groups (His-EGFP and His-EGFP/kin-5-Halo) we infered diffusive behavior $(\alpha=1 \pm 0.1)$ of CNTs. These anomaly parameter served as calculation basis for diffusivity $D_{\alpha}$. It is still to be determined whether the found values of diffusivity $\left(D_{\alpha}=0.2-1 \mathrm{~m}^{2} / \mathrm{s}\right)$ describe the passive diffusive behavior of single CNTs in living syncytial Drosophila embryos entirely and reproducibly. In addition to individual MSDs, we have averaged the MSDs of individual CNTs. However, the averaged MSDs all had a high noise level. Since this noise level was not conducive to further processing, we decided to use individual MSDs as a basis for further dynamic studies. This high noise level was also reflected in the behavior of the velocity correlations Hence, a thorough analysis of the velocity correlations was impeded by the high noise level.

From the individual MSDs we calculated the hydrodynamic length of CNTs in living embryos. Interestingly, this value is in the order of the persistence length of CNTs [179]. For the final clarification of the value of the hydrodynamic length, we recommend the already mentioned (Chap. 7.2 and 7.3 possibility of deep imaging in 3D.

Furthermore, we used our setup to combine CNT imaging and the non-invasive method of PIV] By that we successfully applied these methods to show possible applicabilty to characterization of mesoscopic bulk dynamics in the cytoplasm during phases of enhanced cytoplasmic flow. In this context, we have introduced a possible dynamic order parameter $\phi$ (Chap. 6.4), which can be generalized for 
the characterization of the dynamic phases for further studies (e.g. by extension to cross-correlations). 


\section{Summary and outlook}

In this work, we have invesitgated the optical and dynamical behavior of nearinfrared (NIR) fluorescent carbon nanotubes (CNTs) in Drosophila melanogaster (Drosophila) embryos. We have suspended CNTs in watery solutions by wrapping them single-stranded deoxyribonucleic acid (ssDNA) following and optimizing the protocol as described in chapter 3 . In this chapter, we also described a method leading to successful microinjection of functionalized DNA-wrapped CNTs into syncytial Drosophila embryos. To observe the near-infrared (NIR) fluorescence of CNTs and EGFP-tagged histones, we used a setup enabling the simultaneous use of visible and NIR wide-field fluorescence microscopy as described in chapter 4

In this context, we used nanometer precise $z$-sectioning of the embryo (Chap. 5.2) in order to obtain point spread functions (PSFs) up to a depth of $45 \mu \mathrm{m}$. Furthermore, we found the majority of CNTs to be excitable at $770 \mathrm{~nm}$ which is way beyond the wavelength threshold for autofluorescence excitation, thus allowing background free imaging in the NIR.

In this work, we have used CNTs as near-infrared (NIR) fluorescent probes allowing for tracking of single-nanoparticle movements in the cortex of two particular Drosophila types. The setup has allowed us to record time-scale separated simultaneous multi-channel imaging of NIR CNT fluorescence in conjunction with simultaneous imaging of visible EGFP of individual nuclei during successive developmental stages for $30 \mathrm{~min}$. CNT fluorescence and visible fluorescence of Histone2AvEGFP (His-EGFP) have been imaged for subsequent division cycles. We have captured high frequency information of CNT fluorescence at a rate of $10 \mathrm{~Hz}$, while low frequency dynamics of visible nuclear fluorescence was captured every $20 \mathrm{~s}$ (in case of single-nanoparticle tracking) and every $5 \mathrm{~s}$ (in case of PIV studies) (chapter 6.3). We have successfully applied the open-source software TrackNTrace in order to find individual CNT trajectories. Using custom-written routines for further image postprocessing, we have extracted mean squared displacements (MSDs) and respective anomaly parameters of individual CNTs as well as velocity correlations throughout multiple developmental stages in syncytial Drosophila embryos.

We have compared the diffusive behavior of functionalized NIR fluorescent CNTs in embryos expressing enhanced green fluorescent protein with kinesin-5-HaloTag@ (His-EGFP/kin-5-Halo and to embryos expressing His-EGFP. In both cases we found predominantly sub-diffusive and diffusive behavior of CNTs in the embryos. 
As it turned out, from the available data sets we could not find significant evidence for non-equilibrium behavior based on MSD analysis. We found a hydrodynamic length of $70 \mathrm{~nm}$ for CNTs in Drosophila embryos using MSDs for calculation.

Furthermore, we have demonstrated that a combination of CNT imaging and particle image velocimetry ( $\overline{\mathrm{PIV}}$ ) allows for detection of mesoscopic bulk dynamics in the cytoplasm during phases of enhanced cytoplasmic flow. We achieved this by utilizing our setup, as described in chapter 6.3 in combination with the open-source software PIVIab as well as custom-written software.

To conclude, in this work we have successfully characterized optical properties of single NIR fluorescent CNTs in living Drosophila embryos. Moreover, we were able to profit from the extraordinary fluorescence properties of CNTs for the extraction of single trajectories.

For future research we recommend to extend tracking in Drosophila embryos in three dimensions using the nanometer precise piezo control. Based on our studies, we suggest to elaborate more detailed studies on cytoplasmic flow using a correlation based approach to obtain dynamic order parameters to potentially quantify non-equilibrium contributions to the cytoplasmic flow. Since we have characterized the optical properties of the CNTs that have led to successful imaging in the NIR, we recommend purchasing commercial CNTs with the same properties to further simplify and optimize the handling during sample preparation. In future studies, CNTs can be used to probe locally non-equilibrium dynamics in the actin cortex. 


\section{Appendix}

\section{A.1 Setup components}

\section{A.1.1 Sample preparation}

1. probe tip sonicator: VC $5020 \mathrm{kHz}, 50 \mathrm{~W}$, SONICS \& MATERIALS INC., Danbury, CT, USA

2. small table top centrifuge (100 ml filters): Centrifuge $5417 \mathrm{R}$; Eppendorf AG, Hamburg, Germany

3. large table top centrifuge (4 ml filters): Allegra ${ }^{\circledR}$ X-15R Centrifuge; BECKMANN COULTER, Brea, CA, USA

4. stereo light microscope for embryo alignment: LEICA MZ 6; Leica Camera AG, Wetzlar, Germany

5. microinjector: Transjector 5246 and TransferMan ${ }^{\circledR}$ NK2; Eppendorf AG, Hamburg, Germany

6. stereo light microscope for microinjection: OLYMPUS IX70; Olympus K. K., Tokyo, Japan

7. balance: CP224S; Sartorius AG, Göttingen, Germany

\section{A.1.2 Fluorescence imaging}

1. sample stage; custom built

2. $\alpha$ Plan-APOCHROMAT 100x/1.46 NA Oil DIC (UV) VIS-IR; Zeiss, Göttingen, Germany

3. zt488/532/561/785tpc; Chroma, VT, USA; distributor: AHF, Tübingen, Germany

4. light-tight box; custom built

5. BrightLine ${ }^{\circledR}$ Notch Filter 561; Semrock Inc., Rochester, NY, USA; distributor: AHF, Tübingen, Germany

6. BrightLine ${ }^{\mathrm{R}}$ Notch Filter 488; Semrock Inc., Rochester, NY, USA; distributor: AHF, Tübingen, Germany

7. 452149; Zeiss, Göttingen, Germany

8. 630DCXR; Chroma, VT, USA; distributor: AHF, Tübingen, Germany

9. PF10-03-P01; Thorlabs, Dachau, Germany

10. Acton spectrograph Sp-2150i and InGaAs detector OMA V 512-1.7; Princeton Instruments, Trenton, NJ, USA

11. XEVA-FPA-1.7-320; Xenics, Leuven, Belgium 
12. iXon DU-888E; Andor Technology, Belfast, UK

13. Stanley 111R IR-Diode, AN1111R; Stanley Electric Co., Ltd, Tokyo, Japan

14. NanoCube P611.3S; Physik Instrumente GmbH \& Co. KG, Karlsruhe, Germany

15. M-562-xyz; Newport, Irvine, CA, USA

16. ET LP 900; Chroma, VT, USA; distributor: AHF, Tübingen, Germany

17. FESH0650; Thorlabs, Dachau, Germany

18. ZET 561 NF 242059; Chroma, VT, USA; distributor: AHF, Tübingen, Germany

19. AC254-300-A-ML; Thorlabs, Dachau, Germany

20. AC254-150-A-ML; Thorlabs, Dachau, Germany

21. AC254-150-B; Thorlabs, Dachau, Germany

22. AC254-040-A-ML; Thorlabs, Dachau, Germany

23. AC254-035-B; Thorlabs, Dachau, Germany

24. Cobolt Jive 500, $500 \mathrm{~mW}$; Cobolt, Solna, Sweden

25. MIRA 900-F 710-810 nm; Coherent Inc., Santa Clara, CA, USA

26. $\lambda / 4$ 690-1200nm ACHROMATIC; Thorlabs, Dachau, Germany

27. NDC-25C-2M; Thorlabs, Dachau, Germany

28. 11234858 Beam-Expander; Rodenstock, Munich, Germany

29. E-563 I3N; Physik Instrumente, Karlsruhe, Germany

30. z488RDC; Chroma, VT, USA; distributor: AHF, Tübingen, Germany

31. z561RDC; Chroma, VT, USA; distributor: AHF, Tübingen, Germany

32. OBIS $488 \mathrm{~nm} 50 \mathrm{~mW}$; Coherent Inc., Santa Clara, CA, USA

33. VERDI-V12 532 nm 12 W; Coherent Inc., Santa Clara, CA, USA

34. Cube $637 \mathrm{~nm} 25 \mathrm{~mW}$; Coherent Inc. Santa Clara, CA, USA

35. F21-005; Chroma, VT, USA; distributor: AHF, Tübingen, Germany

36. Plan Apo IR $60 \times / 1.27$ WI OFN25 DIC N2; Nikon, Tokyo, Japan

37. LabMax-TO, S/N 0176A09R; Coherent Inc. Santa Clara, CA, USA

38. E-563 I3N; Physik Instrumente GmbH \& Co. KG, Karlsruhe, Germany 


\section{A.2 Chemical agents and disposables}

\section{A.2.1 Carbon nanotube preparation}

1. HiPCO single-walled carbon nanotubes, batch number 189.2; Rice University, Houston, Texas, USA

2. dT30 AminoC12; Thermo Fisher Scientific Life Technologies GmbH, Darmstadt, Germany

3. spatula: VWR Polypropylene Spatula Eco; VWR ${ }^{\circledR}$ International GmbH, Dresden Germany

4. glass scintillation vial: $4 \mathrm{ml}, 14.75 \times 45 \mathrm{~mm}$, Vials N13 flat Screw Neck 13-425; MACHEREY-NAGEL GmbH \& Co. KG, Düren, Germany

5. HaloTag ${ }^{\circledR}$ Succinimidyl Ester (O4) Ligand; Promega GmbH, Mannheim, Germany

6. Amicon ${ }^{\circledR}$ Ultra $4 \mathrm{ml}$ Centrifugal Filters Ulatracel ${ }^{\circledR}$ 100K; Merck, Molsheim, France

7. Amicon ${ }^{\circledR}$ Ultra 0.5 ml Centrifugal Filters Ulatracel ${ }^{\circledR}$ 10K; Merck, Molsheim, France

8. dimethyl sulfoxide; Sigma-Aldrich Biochemie GmbH, Hamburg, Germany

9. phosphate-buffered saline; Thermo Fisher Scientific Life Technologies GmbH, Darmstadt, Germany

\section{A.2.2 Fluorescence imaging}

1. Immersol ${ }^{\mathrm{TM}} 518 \mathrm{~F}$, Zeiss, Göttingen, Germany

2. FluoroMax ${ }^{\mathrm{TM}}$, R 600, $0.6 \mu \mathrm{m}$; Thermo Fisher Scientific Life Technologies GmbH, Darmstadt, Germany

\section{A.2.3 Fly prepartion and egg collection}

1. Voltalef 10S oil; Lehmann \& Voss, Hamburg, Germany

2. hypochlorite: DanKlorix; CP GABA, Hamburg, Germany

3. glue: Tesa ${ }^{\circledR}$ dissolved in heptane; Beiersdorf, Hamburg, Germany

4. yeast: Backhefe DHW vital gold; Deutsche Hefewerke GmbH, Nuremberg, Germany

5. apple juice: HiPP Bio-Saft Milder Apfel; HiPP Holding, Pfaffenhofen (Ilm), Germany

6. agar: Agar Agar SERVA high-gel strength; SERVA Electrophoresis GmbH, Heidelberg, Germany

7. microinjection needle: Thin Wall Glass Capillaries TW100F-4; World Precision Instruments Germany GmbH, Friedberg, Germany

8. glass coverslip: microscope slides, Menzel-Gläser, $22 \times 40 \mathrm{~mm}$; VWR ${ }^{\circledR}$ International GmbH, Dresden Germany

9. microscope slide: MICROSCOPE SLIDES ECN 631-1550; VWR ${ }^{\circledR}$ International GmbH, Dresden Germany 



\section{Acknowledgements}

I thank Prof. Dr. Christoph F. Schmidt, for his reliable supervision and support, even during his move to Duke University. Despite the large move of his lab, he made sure to make room for phone discussions on a weekly basis.

I also thank Prof. Dr. Stefan Klumpp for correferring my thesis and for being always available for fruitful and targeted discussions and for always having open doors and open ears.

I thank the ERC for financing me over the last three years.

I thank my collaborator Prof. Dr. Jörg Großhans for supportive discussions on biological topics and for discussions on the design of experiments with Drosophila. Further thanks go to Dr. Kengo Nishi, for optimizing the carbon nanotube supsensions used in the studies on Drosophila embryos, presented in this work.

Also, I thank Dr. Zhiyi Lv, for cultivating the Drosophila flies used in our collaboration and for microinjecting carbon nanotube suspensions into Drosophila embryos.

I thank Dieter Hille and his team for discussing the plans on the optomechanics to be built in the imaging setup and for actually crafting the optomechanics.

I also thank Dr. Lautscham, Martin Schönekeß, Simon Hölscher and their team for crafting the electronic parts during the optimization process of the setup. I thank the technical assistants: Tanja Gall, Kerstin von Roden and Ulrike Schulz for introducing me to cell culture and for reliably ordering the biochemical specimens.

I also thank Thomas Geiling for IT support.

I thank the administrative staff: Sabine Huhnold, Nicole Rehbein, Regina Wunderlich and Elke Zech for shielding bureaucracy.

In thank Dr. Simon Stein and Dr. Jan Thiart for providing and introducing me into their particle tracking software TrackNTrace.

I am thankful for the intriguing discussions with Dr. Dieter Klopfenstein on biochemical topics, especially on molecular motors.

I also thank Dr. Florian Rehfeldt for sharing his insights in optics and for giving me the opportunity to gain experience as a time-taker during oral exams.

I appreciate also the discussions about the imaging in Drosophila embryos with my predecessor Dr. Alok Weßel who performed the preliminary studies on carbon nanotubes. 
Thanks go also to our very international lab (eleven nations and counting), which enabled me to deepen my intercultural knowledge and improve my spoken English skills.

I thank my friends during my time in Karlsruhe and the ones I have made in Göttingen: thank you for being there.

Lastly, I thank Friederike and our families for their support during the whole course of the thesis. 


\section{List of Figures}

2.1 Schematic of the cortical region in syncytial Drosophila embryos . . . . 9

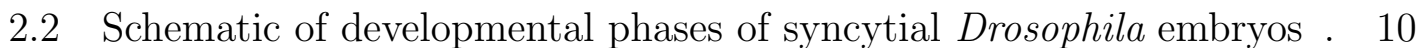

$2.3 \quad$ Schematic of $\mid \mathrm{G} 2$ phase and $\mid \mathrm{M}$ phase $\ldots \ldots \ldots \ldots \ldots \ldots \ldots \ldots \ldots \ldots$

2.4 Schematic of the microscopic picture during cell division . . . . . . . . 13

2.5 Schematic comparing Stokes shifts of CNTs and EGFP ........... . 15

2.6 Schematic comparison of absorption-emission spectra of $\mid$ CNTs $\mid$ and

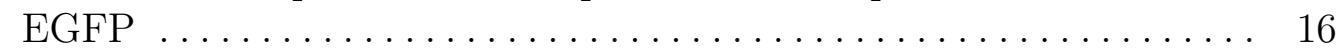

2.7 Schematic of a hexagonal lattice of a graphene sheet $\ldots \ldots \ldots \ldots . .218$

2.8 Schematic of a fluorescence transition $\ldots \ldots \ldots \ldots \ldots \ldots \ldots \ldots \ldots$

2.9 Schematic of the fluorescence transition in a semiconducting [CNT. . . 20

3.1 Schematic of $\mid \mathrm{CNT}$ functionalization $\ldots \ldots \ldots \ldots \ldots \ldots \ldots \ldots \ldots$

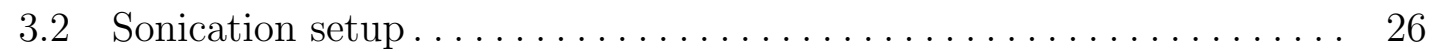

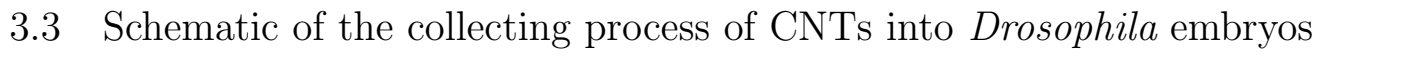
before microinjetion of [CNT suspension $\ldots \ldots \ldots \ldots \ldots \ldots \ldots \ldots$

3.4 Stereo light microscopes for $\mid$ Drosophila $\mid$ embryo microinjection . . . . . 31

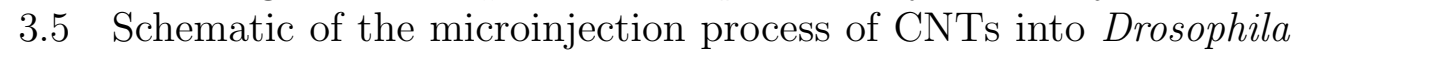

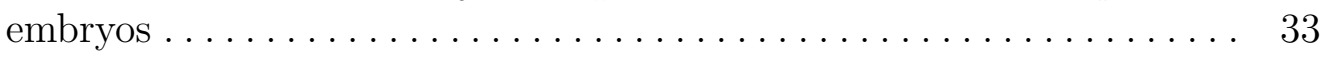

4.1 Schematic of custom-built setup for multimodal NIR and VIS

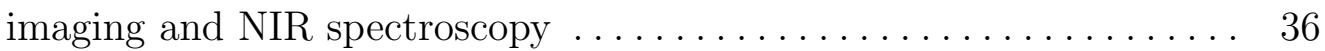

4.2 Setup for multimodal $[\mathrm{NIR}$ and $\mathrm{VIS}$ imaging and IR spectroscopy,

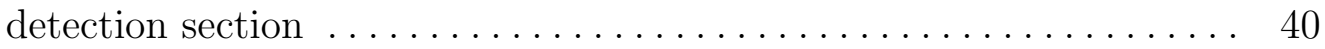

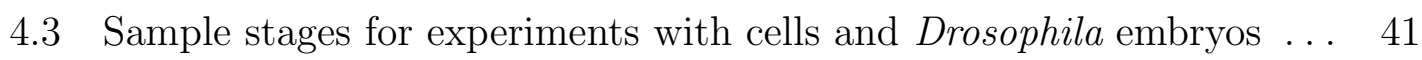

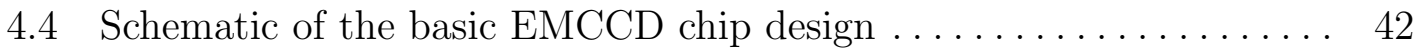

4.5 Resulting spectral peaks, measured by the IR spectrometer (10) for setting the wavelength of the tunable Ti:Sapph] laser (25)] ....... 44

5.1 Comparison of two laser wavelengths to excite CNT] $[$ NIR fluorescence

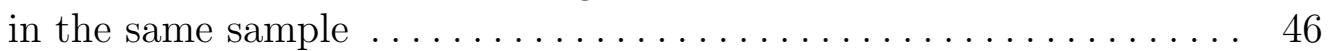

5.2 Schematic of the measuring points of the powers of the lasers for CNT fluorescence excitation in the imaging setup . . . . . . . . . 47

5.3 Schematic of scanning $\mid$ CNTs in $\mid$ Drosophila embryonic tissue. . . . . . . 48

\begin{tabular}{|lll|l|l|}
\hline 5.4 & Experimental data showing the width distribution of & PSFs & of \\
\hline
\end{tabular}

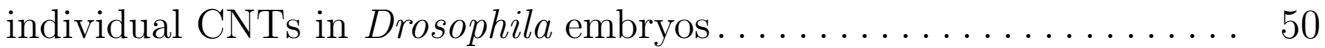


6.1 Generic protocol for simultaneous VIS $\mid$ NIR imaging . . . . . . . . . . 52

6.2 Assembly of CNTs in the vicinity of nuclei in two embryo types. . . . 54

6.3 Schematic of the calibration for parfocality for the multimodal

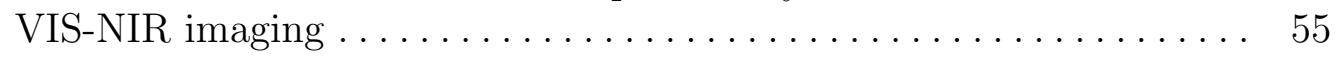

6.4 Bead pattern for [FOV] calibration for 1VISHNIR] imaging . . . . . . . . 56

\begin{tabular}{|ll|l|l|l|}
\hline 6.5 & Recording of His-EGFP in several nuclear division cycles of & Drosophila & 57
\end{tabular}

6.6 Registered images of nuclei in $\mid$ Drosophila $\mid$ embryos and NIR]

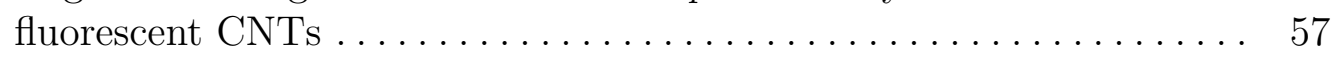

6.7 Exemplary protocol of the registration of the image sequences during the multimodal VISHNIR image sequences $\ldots \ldots \ldots \ldots \ldots \ldots \ldots$

6.8 Exemplary kymographs of individual CNTs in the cortex of a

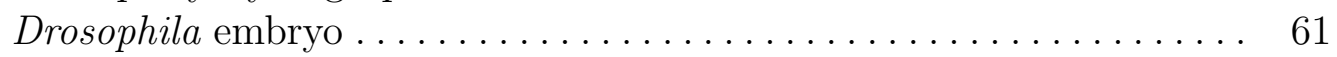

6.9 Trajectories of CNTs in Drosophila embryos . . . . . . . . . . . . . . . 63

6.10 Statistics of track lifetimes of individual CNTs in embryos (His-EGFP)

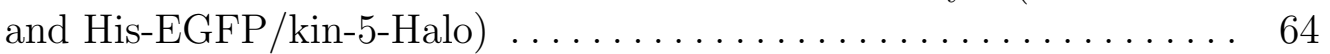

6.11 MSDs of CNTs in embryos (His-EGFP vs. His-EGFP/kin-5-Halo)] . . 65

6.12 Histograms reflecting the anomaly parameters of CNTs in two distinct transgenic crosses of $\mid$ Drosophila $\mid$ embryos at different time

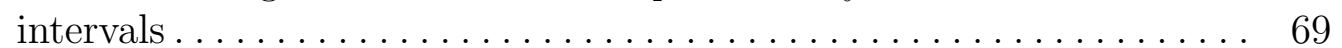

6.13 Statistics of the anomaly parameters $\alpha$ for short and long track lifetimes 70

6.14 Distribution of different error models (MAE, RMSE, nRMSE, $\mid \mathrm{CV}(\mathrm{RMSE}) \boldsymbol{D}$ for power law MSD fits. $\ldots \ldots \ldots \ldots \ldots \ldots \ldots \ldots \ldots \ldots \ldots$

6.15 Scatter plot of the values of the MAE $\cap$ RMSE corrected anomaly

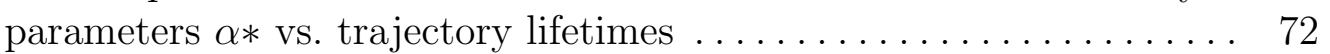

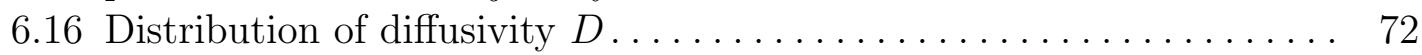

6.17 Velocity correlations in Drosophila embryos (His-EGFP) and

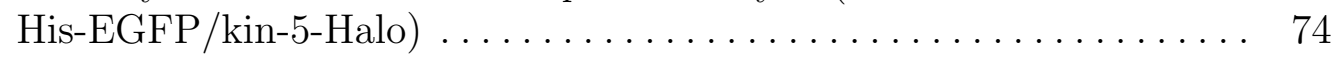

6.18 Cytoplasmic flow in Drosophila $\mid$ embryos obtained from combining

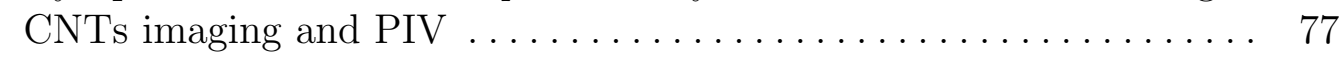




\section{List of Tables}

$4.1 \quad$ Optical components used for multimodal imaging (Fig. 4.1) . . . . . 37

5.1 Excitation laser powers of the green laser $(561 \mathrm{~nm})$ and the Ti:Sapph laser $(770 \mathrm{~nm})$ measured at particular points in the imaging setup. . . 47

6.1 Parameters fed into Micro-Manager for imaging with EMCCD camera 53

6.2 Parameters used for particle tracking with TrackNTrace ......... 62

6.3 Quantiles of four error models $\ldots \ldots \ldots \ldots \ldots \ldots \ldots \ldots \ldots \ldots \ldots \ldots \ldots$ 



\section{References}

[1] T. H. Tan, M. Malik-Garbi, E. Abu-Shah, J. Li, A. Sharma, F. C. MacKintosh, K. Keren, C. F. Schmidt, and N. Fakhri, "Self-organized stress patterns drive state transitions in actin cortices," Science advances, vol. 4, no. 6, p. eaar2847, 2018.

[2] S. Ornes, "Core concept: how nonequilibrium thermodynamics speaks to the mystery of life," Proceedings of the National Academy of Sciences, vol. 114, no. 3, pp. 423-424, 2017.

[3] C. Battle, C. P. Broedersz, N. Fakhri, V. F. Geyer, J. Howard, C. F. Schmidt, and F. C. MacKintosh, "Broken detailed balance at mesoscopic scales in active biological systems," Science, vol. 352, no. 6285, pp. 604-607, 2016.

[4] J. Gladrow, N. Fakhri, F. MacKintosh, C. Schmidt, and C. Broedersz, "Broken detailed balance of filament dynamics in active networks," Physical review letters, vol. 116, no. 24, p. 248301, 2016.

[5] A. D. Wessel, M. Gumalla, J. Grosshans, and C. F. Schmidt, "The mechanical properties of early drosophila embryos measured by high-speed video microrheology," Biophysical journal, vol. 108, no. 8, pp. 1899-1907, 2015.

[6] N. Fakhri, A. D. Wessel, C. Willms, M. Pasquali, D. R. Klopfenstein, F. C. MacKintosh, and C. F. Schmidt, "High-resolution mapping of intracellular fluctuations using carbon nanotubes," Science, vol. 344, no. 6187, pp. 1031-1035, 2014.

[7] C. Veigel and C. F. Schmidt, "Moving into the cell: single-molecule studies of molecular motors in complex environments," Nature Reviews Molecular Cell Biology, vol. 12, no. 3, p. 163, 2011.

[8] N. Fakhri, F. C. MacKintosh, B. Lounis, L. Cognet, and M. Pasquali, "Brownian motion of stiff filaments in a crowded environment," Science, vol. 330, no. 6012, pp. 1804-1807, 2010.

[9] M. Krieg, G. Fläschner, D. Alsteens, B. M. Gaub, W. H. Roos, G. J. Wuite, H. E. Gaub, C. Gerber, Y. F. Dufrêne, and D. J. Müller, "Atomic force microscopy-based mechanobiology," Nature Reviews Physics, p. 1, 2018.

[10] C. Bustamante, "Molecular machines one molecule at a time," Protein science: a publication of the Protein Society, vol. 26, no. 7, p. 1245, 2017.

[11] T. Ha, "Probing natureâĂŹs nanomachines one molecule at a time," Biophysical journal, vol. 110, no. 5, pp. 1004-1007, 2016. 
[12] N. G. Walter and C. Bustamante, "Introduction to single molecule imaging and mechanics: seeing and touching molecules one at a time," 2014.

[13] R. Kasper, B. Harke, C. Forthmann, P. Tinnefeld, S. W. Hell, and M. Sauer, "Fluorophores: Single-molecule sted microscopy with photostable organic fluorophores (small 13/2010)," Small, vol. 6, no. 13, 2010.

[14] A. V. Mamontova, I. D. Solovyev, A. P. Savitsky, A. . Shakhov, K. A. Lukyanov, and A. M. Bogdanov, "Bright gfp with subnanosecond fluorescence lifetime," Scientific reports, vol. 8, 2018.

[15] R. Y. Tsien, "The green fluorescent protein," 1998.

[16] M. Chalfie, Y. Tu, G. Euskirchen, W. W. Ward, and D. C. Prasher, "Green fluorescent protein as a marker for gene expression," Science, vol. 263, no. 5148, pp. 802-805, 1994.

[17] S. Ghosh, S. M. Bachilo, R. A. Simonette, K. M. Beckingham, and R. B. Weisman, "Oxygen doping modifies near-infrared band gaps in fluorescent single-walled carbon nanotubes," Science, vol. 330, no. 6011, pp. 1656-1659, 2010.

[18] D. A. Tsyboulski, E. L. Bakota, L. S. Witus, J.-D. R. Rocha, J. D. Hartgerink, and R. B. Weisman, "Self-assembling peptide coatings designed for highly luminescent suspension of single-walled carbon nanotubes," Journal of the American Chemical Society, vol. 130, no. 50, pp. 17134-17140, 2008.

[19] O. Kiowski, K. Arnold, S. Lebedkin, F. Hennrich, and M. M. Kappes, "Direct observation of deep excitonic states in the photoluminescence spectra of singlewalled carbon nanotubes," Physical review letters, vol. 99, no. 23, p. 237402, 2007.

[20] M. J. O'connell, S. M. Bachilo, C. B. Huffman, V. C. Moore, M. S. Strano, E. H. Haroz, K. L. Rialon, P. J. Boul, W. H. Noon, C. Kittrell, et al., "Band gap fluorescence from individual single-walled carbon nanotubes," Science, vol. 297, no. 5581, pp. 593-596, 2002.

[21] O. Grimm and E. Wieschaus, "The bicoid gradient is shaped independently of nuclei," Development, vol. 137, no. 17, pp. 2857-2862, 2010.

[22] A. C. Martin, M. Kaschube, and E. F. Wieschaus, "Pulsed contractions of an actin-myosin network drive apical constriction," Nature, vol. 457, no. 7228, p. 495, 2009.

[23] D. St Johnston and C. Nüsslein-Volhard, "The origin of pattern and polarity in the drosophila embryo," Cell, vol. 68, no. 2, pp. 201-219, 1992.

[24] E. B. Lewis, "A gene complex controlling segmentation in drosophila," in Genes, Development and Cancer, pp. 205-217, Springer, 1978.

[25] T. Casci, "Development: Drosophila embryos are doing it for themselves," Nature Reviews Genetics, vol. 10, no. 5, p. 279, 2009.

[26] C. Nüsslein-Volhard and E. Wieschaus, "Mutations affecting segment number and polarity in drosophila," Nature, vol. 287, p. 795, 1980.

[27] Z. Lv, "Regulation of actin dynamics by formin in early drosophila embryogenesis," 2014.

[28] A. W. Shermoen and P. H. O'Farrell, "Progression of the cell cycle through mitosis leads to abortion of nascent transcripts," Cell, vol. 67, no. 2, pp. 303-310, 1991. 
[29] J. W. Posakony, J. M. England, and G. Attardi, "Mitochondrial growth and division during the cell cycle in hela cells," The Journal of cell biology, vol. 74, no. 2, pp. 468-491, 1977.

[30] O. Grimm, M. Coppey, and E. Wieschaus, "Modelling the bicoid gradient," Development, vol. 137, no. 14, pp. 2253-2264, 2010.

[31] S. Moody, Cell Lineage and Fate Determination. Academic Press, 1999.

[32] B. Alberts, Essential Cell Biology. Garland Science, 5th ed., 2015.

[33] J. A. Farrell and P. H. O'Farrell, "From egg to gastrula: how the cell cycle is remodeled during the drosophila mid-blastula transition," Annual review of genetics, vol. 48, pp. 269-294, 2014.

[34] L. G. Guilgur, P. Prudêncio, D. Sobral, D. Liszekova, A. Rosa, and R. G. Martinho, "Requirement for highly efficient pre-mrna splicing during drosophila early embryonic development," Elife, vol. 3, p. e02181, 2014.

[35] K. G. Hales, C. A. Korey, A. M. Larracuente, and D. M. Roberts, "Genetics on the fly: a primer on the drosophila model system," Genetics, vol. 201, no. 3, pp. 815-842, 2015.

[36] B. Alberts, A. Johnson, J. Lewis, M. Raff, K. Roberts, and P. Walter, "Drosophila and the molecular genetics of pattern formation: Genesis of the body plan," in Molecular Biology of the Cell. 4th edition, Garland Science, 2002.

[37] P. E. Young, T. C. Pesacreta, and D. P. Kiehart, "Dynamic changes in the distribution of cytoplasmic myosin during drosophila embryogenesis," Development, vol. 111, no. 1, pp. 1-14, 1991.

[38] B. Alberts, Essential Cell Biology. Garland Science, 3rd ed., 2010.

[39] https://en.wikipedia.org/wiki/Mitosis\#/media/File:Mitosis_ Stages.svg, accessed: 25.08.2019; 16:43.

[40] G. Liccardi and L. L. Fava, "Fiat lux: illuminating the cell cycle," 2017.

[41] T. Hunt, K. Nasmyth, and B. Novák, "Introduction: The cell cycle," Philosophical Transactions: Biological Sciences, vol. 366, no. 1584, pp. 3494-3497, 2011.

[42] K. Schafer, "The cell cycle: a review," Veterinary pathology, vol. 35, no. 6, pp. 461-478, 1998.

[43] B. Alberts, Essential cell biology. Garland Science, 3rd ed., 2010.

[44] B. Alberts, Molecular biology of the cell. Garland Science, 6th ed., 2015.

[45] A. Düselder, V. Fridman, C. Thiede, A. Wiesbaum, A. Goldstein, D. R. Klopfenstein, O. Zaitseva, M. E. Janson, L. Gheber, and C. F. Schmidt, "Deletion of the tail domain of the kinesin- 5 cin 8 affects its directionality," Journal of Biological Chemistry, vol. 290, no. 27, pp. 16841-16850, 2015.

[46] C. Thiede, V. Fridman, A. Gerson-Gurwitz, L. Gheber, and C. F. Schmidt, "Regulation of bi-directional movement of single kinesin-5 cin8 molecules," Bioarchitecture, vol. 2, no. 2, pp. 70-74, 2012.

[47] L. C. Kapitein, B. H. Kwok, J. S. Weinger, C. F. Schmidt, T. M. Kapoor, and E. J. Peterman, "Microtubule cross-linking triggers the directional motility of kinesin-5," The Journal of cell biology, vol. 182, no. 3, pp. 421-428, 2008.

[48] B. J. Mann and P. Wadsworth, "Kinesin-5 regulation and function in mitosis," Trends in cell biology, 2018. 
[49] C. L. Hueschen, A. F. Long, and S. Dumont, "Kinesin-5: A team is just the sum of its parts," Developmental cell, vol. 34, no. 6, pp. 609-610, 2015.

[50] Y. Chen and W. O. Hancock, "Kinesin-5 is a microtubule polymerase," Nature communications, vol. 6, p. 8160, 2015.

[51] J. S. Waitzman and S. E. Rice, "Mechanism and regulation of kinesin-5, an essential motor for the mitotic spindle," Biology of the Cell, vol. 106, no. 1, pp. 1-12, 2014.

[52] R. Avunie-Masala, N. Movshovich, Y. Nissenkorn, A. Gerson-Gurwitz, V. Fridman, M. Kõivomägi, M. Loog, M. A. Hoyt, A. Zaritsky, and L. Gheber, "Phospho-regulation of kinesin-5 during anaphase spindle elongation," J Cell Sci, vol. 124, no. 6, pp. 873-878, 2011.

[53] S. A. Endow, F. J. Kull, and H. Liu, "Kinesins at a glance," J Cell Sci, vol. 123, no. 20, pp. 3420-3424, 2010.

[54] N. P. Ferenz, A. Gable, and P. Wadsworth, "Mitotic functions of kinesin-5," in Seminars in cell \& developmental biology, vol. 21, pp. 255-259, Elsevier, 2010.

[55] R. D. Vale, T. Funatsu, D. W. Pierce, L. Romberg, Y. Harada, and T. Yanagida, "Direct observation of single kinesin molecules moving along microtubules," Nature, vol. 380, no. 6573, p. 451, 1996.

[56] V. Hariharan and W. O. Hancock, "Insights into the mechanical properties of the kinesin neck linker domain from sequence analysis and molecular dynamics simulations," Cellular and molecular bioengineering, vol. 2, no. 2, pp. 177-189, 2009 .

[57] D. W. Huang, L. Fanti, D. T. Pak, M. R. Botchan, S. Pimpinelli, and R. Kellum, "Distinct cytoplasmic and nuclear fractions of drosophila heterochromatin protein 1: their phosphorylation levels and associations with origin recognition complex proteins," The Journal of cell biology, vol. 142, no. 2, pp. 307-318, 1998.

[58] K. Nishizawa, K. Fujiwara, M. Ikenaga, N. Nakajo, M. Yanagisawa, and D. Mizuno, "Universal glass-forming behavior of in vitro and living cytoplasm," Scientific reports, vol. 7, no. 1, p. 15143, 2017.

[59] R. E. Goldstein and J.-W. van de Meent, "A physical perspective on cytoplasmic streaming," Interface focus, vol. 5, no. 4, p. 20150030, 2015.

[60] K. Doubrovinski, M. Swan, O. Polyakov, and E. F. Wieschaus, "Measurement of cortical elasticity in drosophila melanogaster embryos using ferrofluids," Proceedings of the National Academy of Sciences, vol. 114, no. 5, pp. 10511056, 2017.

[61] M. Guo, A. J. Ehrlicher, M. H. Jensen, M. Renz, J. R. Moore, R. D. Goldman, J. Lippincott-Schwartz, F. C. Mackintosh, and D. A. Weitz, "Probing the stochastic, motor-driven properties of the cytoplasm using force spectrum microscopy," Cell, vol. 158, no. 4, pp. 822-832, 2014.

[62] J.-F. Berret, "Local viscoelasticity of living cells measured by rotational magnetic spectroscopy," Nature communications, vol. 7, p. 10134, 2016.

[63] S. Ganguly, L. S. Williams, I. M. Palacios, and R. E. Goldstein, "Cytoplasmic streaming in drosophila oocytes varies with kinesin activity and correlates with the microtubule cytoskeleton architecture," Proceedings of the National Academy of Sciences, vol. 109, no. 38, pp. 15109-15114, 2012. 
[64] F. Kaiser, Z. Lv, D. M. Rodrigues, J. Rosenbaum, T. Aspelmeier, J. Großhans, and K. Alim, "Mechanical model of nuclei ordering in drosophila embryos reveals dilution of stochastic forces," Biophysical journal, vol. 114, no. 7, pp. 1730-1740, 2018.

[65] L. R. Serbus, B.-J. Cha, W. E. Theurkauf, and W. M. Saxton, "Dynein and the actin cytoskeleton control kinesin-driven cytoplasmic streaming in drosophila oocytes," Development, vol. 132, no. 16, pp. 3743-3752, 2005.

[66] S. Iijima, "Helical microtubules of graphitic carbon," nature, vol. 354, no. 6348, p. 56, 1991.

[67] D. A. Tsyboulski, J.-D. R. Rocha, S. M. Bachilo, L. Cognet, and R. B. Weisman, "Structure-dependent fluorescence efficiencies of individual single-walled carbon nanotubes," Nano letters, vol. 7, no. 10, pp. 3080-3085, 2007.

[68] A. Hartschuh, H. N. Pedrosa, L. Novotny, and T. D. Krauss, "Simultaneous fluorescence and raman scattering from single carbon nanotubes," Science, vol. 301, no. 5638, pp. 1354-1356, 2003.

[69] J. C. Crocker and B. D. Hoffman, "Multiple-particle tracking and two-point microrheology in cells," Methods in cell biology, vol. 83, pp. 141-178, 2007.

[70] Y. Tseng, T. P. Kole, and D. Wirtz, "Micromechanical mapping of live cells by multiple-particle-tracking microrheology," Biophysical journal, vol. 83, no. 6, pp. 3162-3176, 2002.

[71] J. K. Streit, S. M. Bachilo, A. V. Naumov, C. Khripin, M. Zheng, and R. B. Weisman, "Measuring single-walled carbon nanotube length distributions from diffusional trajectories," Acs Nano, vol. 6, no. 9, pp. 8424-8431, 2012.

[72] G. Chen, Y. Seki, H. Kimura, S. Sakurai, M. Yumura, K. Hata, and D. N. Futaba, "Diameter control of single-walled carbon nanotube forests from 1.3-3.0 nm by arc plasma deposition," Scientific reports, vol. 4, p. 3804, 2014.

[73] J.-D. R. Rocha, S. M. Bachilo, S. Ghosh, S. Arepalli, and R. B. Weisman, "Efficient spectrofluorimetric analysis of single-walled carbon nanotube samples," Analytical chemistry, vol. 83, no. 19, pp. 7431-7437, 2011.

[74] R. B. Weisman and S. M. Bachilo, "Dependence of optical transition energies on structure for single-walled carbon nanotubes in aqueous suspension: an empirical kataura plot," Nano Letters, vol. 3, no. 9, pp. 1235-1238, 2003.

[75] M. Pimenta, A. Marucci, S. Empedocles, M. Bawendi, E. Hanlon, A. Rao, P. Eklund, R. Smalley, G. Dresselhaus, and M. Dresselhaus, "Raman modes of metallic carbon nanotubes," Physical Review B, vol. 58, no. 24, p. R16016, 1998.

[76] S. Kruss, D. P. Salem, L. Vuković, B. Lima, E. Vander Ende, E. S. Boyden, and M. S. Strano, "High-resolution imaging of cellular dopamine efflux using a fluorescent nanosensor array," Proceedings of the National Academy of Sciences, vol. 114, no. 8, pp. 1789-1794, 2017.

[77] S. Kruss, A. J. Hilmer, J. Zhang, N. F. Reuel, B. Mu, and M. S. Strano, "Carbon nanotubes as optical biomedical sensors," Advanced drug delivery reviews, vol. 65, no. 15, pp. 1933-1950, 2013.

[78] E. Thimsen, B. Sadtler, and M. Y. Berezin, "Shortwave-infrared (swir) emitters for biological imaging: a review of challenges and opportunities," Nanophotonics, vol. 6, no. 5, pp. 1043-1054, 2017. 
[79] M. Yudasaka, Y. Yomogida, M. Zhang, T. Tanaka, M. Nakahara, N. Kobayashi, Y. Okamatsu-Ogura, K. Machida, K. Ishihara, K. Saeki, et al., "Near-infrared photoluminescent carbon nanotubes for imaging of brown fat," Scientific reports, vol. 7, p. 44760, 2017.

[80] H. Zhang, D. C. Salo, D. M. Kim, S. Komarov, Y.-C. Tai, and M. Y. Berezin, "Penetration depth of photons in biological tissues from hyperspectral imaging in shortwave infrared in transmission and reflection geometries," Journal of biomedical optics, vol. 21, no. 12, p. 126006, 2016.

[81] S. L. Jacques, "Optical properties of biological tissues: a review," Physics in Medicine \&f Biology, vol. 58, no. 11, p. R37, 2013.

[82] K. Welsher, S. P. Sherlock, and H. Dai, "Deep-tissue anatomical imaging of mice using carbon nanotube fluorophores in the second near-infrared window," Proceedings of the National Academy of Sciences, vol. 108, no. 22, pp. 89438948, 2011.

[83] J. T. Robinson, K. Welsher, S. M. Tabakman, S. P. Sherlock, H. Wang, R. Luong, and H. Dai, "High performance in vivo near-ir $(>1 \mu \mathrm{m})$ imaging and photothermal cancer therapy with carbon nanotubes," Nano research, vol. 3, no. 11, pp. 779-793, 2010.

[84] K. Welsher, Z. Liu, S. P. Sherlock, J. T. Robinson, Z. Chen, D. Daranciang, and H. Dai, "A route to brightly fluorescent carbon nanotubes for near-infrared imaging in mice," Nature nanotechnology, vol. 4, no. 11, p. 773, 2009.

[85] P. Cherukuri, C. J. Gannon, T. K. Leeuw, H. K. Schmidt, R. E. Smalley, S. A. Curley, and R. B. Weisman, "Mammalian pharmacokinetics of carbon nanotubes using intrinsic near-infrared fluorescence," Proceedings of the National Academy of Sciences, vol. 103, no. 50, pp. 18882-18886, 2006.

[86] Z. Gao, Y. Hao, M. Zheng, and Y. Chen, "A fluorescent dye with large stokes shift and high stability: synthesis and application to live cell imaging," Rsc Advances, vol. 7, no. 13, pp. 7604-7609, 2017.

[87] J. Mu, Y. Ma, H. Yin, C. Liu, and M. Rohlfing, "Photoluminescence of singlewalled carbon nanotubes: The role of stokes shift and impurity levels," Physical review letters, vol. 111, no. 13, p. 137401, 2013.

[88] S. M. Bachilo, M. S. Strano, C. Kittrell, R. H. Hauge, R. E. Smalley, and R. B. Weisman, "Structure-assigned optical spectra of single-walled carbon nanotubes," Science, vol. 298, no. 5602, pp. 2361-2366, 2002.

[89] M. Monici, "Cell and tissue autofluorescence research and diagnostic applications," Biotechnology annual review, vol. 11, pp. 227-256, 2005.

[90] M. A. Hink, R. A. Griep, J. W. Borst, A. Van Hoek, M. H. Eppink, A. Schots, and A. J. Visser, "Structural dynamics of green fluorescent protein alone and fused with a single chain fv protein," Journal of Biological Chemistry, vol. 275, no. 23, pp. 17556-17560, 2000.

[91] S. Berciaud, L. Cognet, and B. Lounis, "Luminescence decay and the absorption cross section of individual single-walled carbon nanotubes," Physical Review Letters, vol. 101, no. 7, p. 077402, 2008.

[92] F. Wang, G. Dukovic, L. E. Brus, and T. F. Heinz, "Time-resolved fluorescence of carbon nanotubes and its implication for radiative lifetimes," Physical review letters, vol. 92, no. 17, p. 177401, 2004. 
[93] R. B. Weisman, "Fluorimetric characterization of single-walled carbon nanotubes," Analytical and bioanalytical chemistry, vol. 396, no. 3, pp. 1015-1023, 2010.

[94] L. J. Carlson, S. E. Maccagnano, M. Zheng, J. Silcox, and T. D. Krauss, "Fluorescence efficiency of individual carbon nanotubes," Nano letters, vol. 7, no. 12, pp. 3698-3703, 2007.

[95] L. Cognet, D. A. Tsyboulski, J.-D. R. Rocha, C. D. Doyle, J. M. Tour, and R. B. Weisman, "Stepwise quenching of exciton fluorescence in carbon nanotubes by single-molecule reactions," Science, vol. 316, no. 5830, pp. 1465-1468, 2007.

[96] D. A. Tsyboulski, S. M. Bachilo, and R. B. Weisman, "Versatile visualization of individual single-walled carbon nanotubes with near-infrared fluorescence microscopy," Nano letters, vol. 5, no. 5, pp. 975-979, 2005.

[97] K. Matsuda, Y. Kanemitsu, K. Irie, T. Saiki, T. Someya, S. Miyauchi, and S. Maruyama, "Photoluminescence intermittency in an individual single-walled carbon nanotube at room temperature," Applied Physics Letters, vol. 86, no. 12, p. 123116, 2005.

[98] D. A. Heller, S. Baik, T. E. Eurell, and M. S. Strano, "Single-walled carbon nanotube spectroscopy in live cells: towards long-term labels and optical sensors," Advanced Materials, vol. 17, no. 23, pp. 2793-2799, 2005.

[99] A. Jorio, A. Souza Filho, G. Dresselhaus, M. Dresselhaus, A. Swan, M. Ünlü, B. Goldberg, M. Pimenta, J. Hafner, C. Lieber, et al., "G-band resonant raman study of 62 isolated single-wall carbon nanotubes," Physical Review B, vol. 65, no. 15 , p. $155412,2002$.

[100] S. Brown, A. Jorio, a. P. Corio, M. Dresselhaus, G. Dresselhaus, R. Saito, and K. Kneipp, "Origin of the breit-wigner-fano lineshape of the tangential g-band feature of metallic carbon nanotubes," Physical Review B, vol. 63, no. 15, p. 155414, 2001.

[101] M. Dresselhaus and P. Eklund, "Phonons in carbon nanotubes," Advances in Physics, vol. 49, no. 6, pp. 705-814, 2000.

[102] M. Pimenta, A. Marucci, S. Brown, M. Matthews, A. Rao, P. Eklund, R. Smalley, G. Dresselhaus, and M. Dresselhaus, "Resonant raman effect in single-wall carbon nanotubes," Journal of materials research, vol. 13, no. 9, pp. 2396-2404, 1998.

[103] A. R. Amori, Z. Hou, and T. D. Krauss, "Excitons in single-walled carbon nanotubes and their dynamics," Annual review of physical chemistry, vol. 69, pp. 81-99, 2018.

[104] F. Wang, D. J. Cho, B. Kessler, J. Deslippe, P. J. Schuck, S. G. Louie, A. Zettl, T. F. Heinz, and Y. R. Shen, "Observation of excitons in one-dimensional metallic single-walled carbon nanotubes," Physical review letters, vol. 99, no. 22, p. 227401, 2007.

[105] C. Thomsen, H. Telg, J. Maultzsch, and S. Reich, "Chirality assignments in carbon nanotubes based on resonant raman scattering," physica status solidi (b), vol. 242, no. 9, pp. 1802-1806, 2005.

[106] A. Jorio, M. S. Dresselhaus, R. Saito, and G. Dresselhaus, Raman spectroscopy in graphene related systems. John Wiley \& Sons, 2011.

[107] P. Atkfins, Physikalische Chemie. Wiley-VCH Verlag, 4th ed., 2006. 
[108] P. Atkfins, Physikalische Chemie. Wiley-VCH Verlag, 4th ed., 2006.

[109] J. Crochet, M. Clemens, and T. Hertel, "Quantum yield heterogeneities of aqueous single-wall carbon nanotube suspensions," Journal of the American Chemical Society, vol. 129, no. 26, pp. 8058-8059, 2007.

[110] Y. Matsuda, J. Tahir-Kheli, and W. A. Goddard III, "Definitive band gaps for single-wall carbon nanotubes," The Journal of Physical Chemistry Letters, vol. 1, no. 19, pp. 2946-2950, 2010.

[111] G. Dukovic, F. Wang, D. Song, M. Y. Sfeir, T. F. Heinz, and L. E. Brus, "Structural dependence of excitonic optical transitions and band-gap energies in carbon nanotubes," Nano letters, vol. 5, no. 11, pp. 2314-2318, 2005.

[112] T. Nishihara, Y. Yamada, M. Okano, and Y. Kanemitsu, "Dynamics of the lowest-energy excitons in single-walled carbon nanotubes under resonant and nonresonant optical excitation," The Journal of Physical Chemistry C, vol. 119, no. 51, pp. 28654-28659, 2015.

[113] D. Y. Joh, J. Kinder, L. H. Herman, S.-Y. Ju, M. A. Segal, J. N. Johnson, G. K.-L. Chan, and J. Park, "Single-walled carbon nanotubes as excitonic optical wires," Nature nanotechnology, vol. 6, no. 1, p. 51, 2011.

[114] J. Lefebvre and P. Finnie, "Excited excitonic states in single-walled carbon nanotubes," Nano letters, vol. 8, no. 7, pp. 1890-1895, 2008.

[115] R. Pomraenke, J. Maultzsch, S. Reich, E. Chang, D. Prezzi, A. Ruini, E. Molinari, M. Strano, C. Thomsen, and C. Lienau, "Two-photon photoluminescence and exciton binding energies in single-walled carbon nanotubes," physica status solidi (b), vol. 243, no. 10, pp. 2428-2435, 2006.

[116] J. Maultzsch, R. Pomraenke, S. Reich, E. Chang, D. Prezzi, A. Ruini, E. Molinari, M. Strano, C. Thomsen, and C. Lienau, "Exciton binding energies in carbon nanotubes from two-photon photoluminescence," Physical Review B, vol. 72, no. 24, p. 241402, 2005.

[117] F. Wang, G. Dukovic, L. E. Brus, and T. F. Heinz, "The optical resonances in carbon nanotubes arise from excitons," Science, vol. 308, no. 5723, pp. 838-841, 2005.

[118] Y.-Z. Ma, L. Valkunas, S. M. Bachilo, and G. R. Fleming, "Exciton binding energy in semiconducting single-walled carbon nanotubes," The Journal of Physical Chemistry B, vol. 109, no. 33, pp. 15671-15674, 2005.

[119] J. Lefebvre, D. G. Austing, J. Bond, and P. Finnie, "Photoluminescence imaging of suspended single-walled carbon nanotubes," Nano letters, vol. 6, no. 8, pp. 1603-1608, 2006.

[120] H. Kataura, Y. Kumazawa, Y. Maniwa, I. Umezu, S. Suzuki, Y. Ohtsuka, and Y. Achiba, "Optical properties of single-wall carbon nanotubes," Synthetic metals, vol. 103, no. 1-3, pp. 2555-2558, 1999.

[121] A. Alam, S. Dehm, F. Hennrich, Y. Zakharko, A. Graf, M. Pfohl, I. M. Hossain, M. M. Kappes, J. Zaumseil, R. Krupke, et al., "Photocurrent spectroscopy of dye-sensitized carbon nanotubes," Nanoscale, vol. 9, no. 31, pp. 11205-11213, 2017.

[122] A. Setaro, "Advanced carbon nanotubes functionalization," Journal of Physics: Condensed Matter, vol. 29, no. 42, p. 423003, 2017. 
[123] S. Cambré, P. Muyshondt, R. Federicci, and W. Wenseleers, "Chiralitydependent densities of carbon nanotubes by in situ 2d fluorescence-excitation and raman characterisation in a density gradient after ultracentrifugation," Nanoscale, vol. 7, no. 47, pp. 20015-20024, 2015.

[124] K. Mulla, S. Liang, H. Shaik, E. A. Younes, A. Adronov, and Y. Zhao, "Dithiafulvenyl-grafted phenylene ethynylene polymers as selective and reversible dispersants for single-walled carbon nanotubes," Chemical Communications, vol. 51, no. 1, pp. 149-152, 2015.

[125] K. Umemura, "Hybrids of nucleic acids and carbon nanotubes for nanobiotechnology," Nanomaterials, vol. 5, no. 1, pp. 321-350, 2015.

[126] J. Sim, H. Oh, E. Koo, and S.-Y. Ju, "Effect of tight flavin mononucleotide wrapping and its binding affinity on carbon nanotube covalent reactivities," Physical Chemistry Chemical Physics, vol. 15, no. 44, pp. 19169-19179, 2013.

[127] Y. Yu, V. Pushparaj, O. Nalamasu, and L. McGown, "G-quadruplex guanosine gels and single walled carbon nanotubes," Molecules, vol. 18, no. 12, pp. 1543415447, 2013.

[128] H. Li and Q. Li, "Selective separation of single-walled carbon nanotubes in solution," Electronic Properties of Carbon Nanotubes, pp. 69-91, 2011.

[129] N. Nakashima, "Determination of redox states of (n, m) single-walled carbon nanotubes," in 2011 IEEE Nanotechnology Materials and Devices Conference, pp. 6-9, IEEE, 2011.

[130] T. Fujigaya and N. Nakashima, "Single-walled carbon nanotubes as a molecular heater for thermoresponsive polymer gel composite," in Carbon NanotubesPolymer Nanocomposites, IntechOpen, 2011.

[131] S.-K. Joung, T. Okazaki, S. Okada, and S. Iijima, "Interaction between singlewall carbon nanotubes and encapsulated c 60 probed by resonance raman spectroscopy," Physical Chemistry Chemical Physics, vol. 12, no. 28, pp. 81198123, 2010.

[132] S. Maruyama, Y. Miyauchi, Y. Murakami, and S. Chiashi, "Optical characterization of single-walled carbon nanotubes synthesized by catalytic decomposition of alcohol," New Journal of Physics, vol. 5, no. 1, p. 149, 2003.

[133] A. D. Weß el, "Intracellular micromechanics of the syncytial drosophila embryo," 2015.

[134] J. J. Crochet, J. D. Sau, J. G. Duque, S. K. Doorn, and M. L. Cohen, "Electrodynamic and excitonic intertube interactions in semiconducting carbon nanotube aggregates," ACS nano, vol. 5, no. 4, pp. 2611-2618, 2011.

[135] Rudolf Gross, Achim Marx, Festkoerperphysik. Oldenbourg Verlag Munich, 2012.

[136] L. Van Hove, "The occurrence of singularities in the elastic frequency distribution of a crystal," Physical Review, vol. 89, no. 6, p. 1189, 1953.

[137] G. V. Los, L. P. Encell, M. G. McDougall, D. D. Hartzell, N. Karassina, C. Zimprich, M. G. Wood, R. Learish, R. F. Ohana, M. Urh, et al., "Halotag: a novel protein labeling technology for cell imaging and protein analysis," ACS chemical biology, vol. 3, no. 6, pp. 373-382, 2008.

[138] A. I. Zhbanov, E. G. Pogorelov, and Y.-C. Chang, "Van der waals interaction between two crossed carbon nanotubes," Acs Nano, vol. 4, no. 10, pp. 5937-5945, 
2010 .

[139] M. Zheng, A. Jagota, E. D. Semke, B. A. Diner, R. S. McLean, S. R. Lustig, R. E. Richardson, and N. G. Tassi, "Dna-assisted dispersion and separation of carbon nanotubes," Nature materials, vol. 2, no. 5, p. 338, 2003.

[140] Z. Liang, R. Lao, J. Wang, Y. Liu, L. Wang, Q. Huang, S. Song, G. Li, and C. Fan, "Solubilization of single-walled carbon nanotubes with single-stranded dna generated from asymmetric pcr," International journal of molecular sciences, vol. 8, no. 7, pp. 705-713, 2007.

[141] Z. Li, Y. Song, A. Li, W. Xu, and W. Zhang, "Direct observation of the wrapping/unwrapping of ssdna around/from a swcnt at the single-molecule level: towards tuning the binding mode and strength," Nanoscale, vol. 10, no. 39, pp. 18586-18596, 2018.

[142] B. Koh and W. Cheng, "The impact of sonication on the surface quality of single-walled carbon nanotubes," Journal of pharmaceutical sciences, vol. 104, no. 8, pp. 2594-2599, 2015.

[143] K. Yang, Z. Yi, Q. Jing, R. Yue, W. Jiang, and D. Lin, "Sonication-assisted dispersion of carbon nanotubes in aqueous solutions of the anionic surfactant sdbs: The role of sonication energy," Chinese science bulletin, vol. 58, no. 17, pp. 2082-2090, 2013.

[144] A. D. Edelstein, M. A. Tsuchida, N. Amodaj, H. Pinkard, R. D. Vale, and N. Stuurman, "Advanced methods of microscope control using $\mu$ manager software," Journal of biological methods, vol. 1, no. 2, 2014.

[145] W. S. Boyle and G. E. Smith, "Charge coupled semiconductor devices," Bell System Technical Journal, vol. 49, no. 4, pp. 587-593, 1970.

[146] G. Amelio, M. Tompsett, and G. Smith, "Experimental verification of the charge coupled device concept," Bell System Technical Journal, vol. 49, no. 4, pp. 593-600, 1970.

[147] A. E. Spinelli, C. R. Gigliotti, and F. Boschi, "Unified approach for bioluminescence, cerenkov, $\beta$, $\mathrm{x}$ and $\gamma$ rays imaging," Biomedical optics express, vol. 6 , no. 6, pp. 2168-2180, 2015.

[148] M. S. Robbins and B. J. Hadwen, "The noise performance of electron multiplying charge-coupled devices," IEEE transactions on electron devices, vol. 50, no. 5, pp. 1227-1232, 2003.

[149] S. Yokogawa, I. Oshiyama, H. Ikeda, Y. Ebiko, T. Hirano, S. Saito, T. Oinoue, Y. Hagimoto, and H. Iwamoto, "Ir sensitivity enhancement of cmos image sensor with diffractive light trapping pixels," Scientific reports, vol. 7, no. 1, p. 3832, 2017.

[150] T. Pearsall, "Ga 0.47 in 0.53 as: A ternary semiconductor for photodetector applications," IEEE Journal of Quantum Electronics, vol. 16, no. 7, pp. 709-720, 1980.

[151] D. Litwiller, "Ccd vs. cmos," Photonics spectra, vol. 35, no. 1, pp. 154-158, 2001.

[152] https://www.analog.com/en/technical-articles/ paralleling-amplifiers-improves-signal-to-noise-performance. html, accessed: 19.08.2019; 15:53.

[153] https://tinyurl.com/yyeog4qg, accessed: 16.08.2019; 19:54. 
[154] M. Lehmann, C. Wittpahl, H. B. Zakour, and A. Braun, "Resolution and accuracy of non-linear regression of psf with artificial neural networks," in Optical Instrument Science, Technology, and Applications, vol. 10695, p. 106950C, International Society for Optics and Photonics, 2018.

[155] J. Schindelin, I. Arganda-Carreras, E. Frise, V. Kaynig, M. Longair, T. Pietzsch, S. Preibisch, C. Rueden, S. Saalfeld, B. Schmid, et al., "Fiji: an open-source platform for biological-image analysis," Nature methods, vol. 9, no. 7, p. 676, 2012.

[156] L. Su, G. Lu, B. Kenens, S. Rocha, E. Fron, H. Yuan, C. Chen, P. Van Dorpe, M. B. Roeffaers, H. Mizuno, et al., "Visualization of molecular fluorescence point spread functions via remote excitation switching fluorescence microscopy," Nature communications, vol. 6, p. 6287, 2015.

[157] H. Kirshner, F. Aguet, D. Sage, and M. Unser, "3-d psf fitting for fluorescence microscopy: implementation and localization application," Journal of microscopy, vol. 249, no. 1, pp. 13-25, 2013.

[158] M. de Moraes Marim, B. Zhang, J.-C. Olivo-Marin, and C. Zimmer, "Improving single particle localization with an empirically calibrated gaussian kernel," in 2008 5th IEEE International Symposium on Biomedical Imaging: From Nano to Macro, pp. 1003-1006, IEEE, 2008.

[159] T. Arens, F. Hettlic, C. Karpfinger, U. Kockelkorn, K. Lichtenegger, and H. Stachel, Mathematik. Spektrum Akademischer Verlag Heidelberg, 2009.

[160] A. Edelstein, N. Amodaj, K. Hoover, R. Vale, and N. Stuurman, "Computer control of microscopes using $\mu$ manager," Current protocols in molecular biology, vol. 92, no. 1, pp. 14-20, 2010.

[161] O. Shimomura, "The discovery of aequorin and green fluorescent protein," Journal of microscopy, vol. 217, no. 1, pp. 3-15, 2005.

[162] M. Ormö, A. B. Cubitt, K. Kallio, L. A. Gross, R. Y. Tsien, and S. J. Remington, "Crystal structure of the aequorea victoria green fluorescent protein," Science, vol. 273, no. 5280, pp. 1392-1395, 1996.

[163] C. M. Wood, J. M. Nicholson, S. J. Lambert, L. Chantalat, C. D. Reynolds, and J. P. Baldwin, "High-resolution structure of the native histone octamer," Acta Crystallographica Section F: Structural Biology and Crystallization Communications, vol. 61, no. 6, pp. 541-545, 2005.

[164] A. M. Saunders, J. Powers, S. Strome, and W. M. Saxton, "Kinesin-5 acts as a brake in anaphase spindle elongation," Current Biology, vol. 17, no. 12, pp. R453-R454, 2007.

[165] S. C. Stein and J. Thiart, "Trackntrace: A simple and extendable open-source framework for developing single-molecule localization and tracking algorithms," Scientific reports, vol. 6, p. 37947, 2016.

[166] X. Michalet, "Mean square displacement analysis of single-particle trajectories with localization error: Brownian motion in an isotropic medium," Physical Review E, vol. 82, no. 4, p. 041914, 2010.

[167] M. Marseguerra and A. Zoia, "Some insights in superdiffusive transport," Physica A: Statistical Mechanics and its Applications, vol. 377, no. 1, pp. 1-14, 2007. 
[168] R. Metzler and J. Klafter, "The restaurant at the end of the random walk: recent developments in the description of anomalous transport by fractional dynamics," Journal of Physics A: Mathematical and General, vol. 37, no. 31, p. R161, 2004.

[169] V. A. Hackley and J. D. Clogston, "Measuring the hydrodynamic size of nanoparticles in aqueous media using batch-mode dynamic light scattering," in Characterization of Nanoparticles Intended for Drug Delivery, pp. 35-52, Springer, 2011.

[170] M. Doi and S. Edwards, The Theory of Polymer Dynamics. Oxford University Press, 1986.

[171] J. Stetefeld, S. A. McKenna, and T. R. Patel, "Dynamic light scattering: a practical guide and applications in biomedical sciences," Biophysical reviews, vol. 8, no. 4, pp. 409-427, 2016.

[172] C. A. Silvera Batista, M. Zheng, C. Y. Khripin, X. Tu, and J. A. Fagan, "Rod hydrodynamics and length distributions of single-wall carbon nanotubes using analytical ultracentrifugation," Langmuir, vol. 30, no. 17, pp. 4895-4904, 2014.

[173] M. Doi and S. Edwards, The Theory of Polymer Dynamics. Oxford University Press, 1986.

[174] A. Melling, "Tracer particles and seeding for particle image velocimetry," Measurement Science and Technology, vol. 8, no. 12, p. 1406, 1997.

[175] W. Thielicke and E. Stamhuis, "Pivlab-towards user-friendly, affordable and accurate digital particle image velocimetry in matlab," Journal of Open Research Software, vol. 2, no. 1, 2014.

[176] W. Thielicke, The flapping flight of birds: Analysis and application. University of Groningen, 2014.

[177] W. Thielicke and E. Stamhuis, "PivlabâĂ ̌̌time-resolved digital particle image velocimetry tool for matlab,(2010)," 2013.

[178] J.-Y. Tinevez, N. Perry, J. Schindelin, G. M. Hoopes, G. D. Reynolds, E. Laplantine, S. Y. Bednarek, S. L. Shorte, and K. W. Eliceiri, "Trackmate: An open and extensible platform for single-particle tracking," Methods, vol. 115, pp. 80-90, 2017.

[179] N. Fakhri, D. A. Tsyboulski, L. Cognet, R. B. Weisman, and M. Pasquali, "Diameter-dependent bending dynamics of single-walled carbon nanotubes in liquids," Proceedings of the National Academy of Sciences, vol. 106, no. 34, pp. 14219-14223, 2009. 


\section{Peer-reviewed journal publication related to this thesis}

Near-infrared fluorescence imaging of single-walled carbon nanotubes in living organisms

Constantin D. C. Kohl, Alok D. Weßel, Kengo Nishi, Zhiyi Lv, Jörg Großhans, Christoph F. Schmidt

submitted to Review of Scientific Instruments. 
